\title{
3D Bipedal Robotic Walking: Models, Feedback Control, and Open Problems
}

\author{
J.W. Grizzle* Christine Chevallereau ** \\ Aaron D. Ames and Ryan W. Sinnet ${ }^{* * *}$ \\ * Control Systems Laboratory, EECS Department, University of \\ Michigan,USA, (email: grizzle@umich.edu) \\ ** CNRS, IRCCyN, Nantes Atlantic University, France, \\ (email: Christine.Chevallereau@irccyn.ec-nantes.fr) \\ *** Department of Mechanical Engineering, Texas A\&M University, \\ USA (email: \{aames,rsinnet\}@tamu.edu)
}

\begin{abstract}
The fields of control and robotics are contributing to the development of bipedal robots that can realize walking motions with the stability and agility of a human being. Dynamic models for bipeds are hybrid in nature. They contain both continuous and discrete elements, with switching events that are spatially driven by unilateral constraints at ground contact and impulse-like forces that occur at foot touchdown. Control laws for these machines must be hybrid as well. The goals of this paper are threefold: highlight certain properties of the models which greatly influence the control law design; present two control design approaches; and indicate some of the many open problems.
\end{abstract}

\section{INTRODUCTION}

This tutorial paper seeks to provide control researchers with an entry point into the area of bipedal locomotion, more specifically, 3D bipedal walking. The emphasis is on models and control laws for achieving the simplest possible behavior, namely, asymptotically stable, periodic, walking on flat ground. This is already a very challenging and rich problem due to the multi-phase, hybrid nature of legged locomotion and the unilateral constraints that must be satisfied by the forces and torques at the footground interface. Any researcher mastering this basic problem can be assured that there is plenty more to do in terms of investigating aperiodic gaits, non-flat ground, maneuvering, running, energy efficiency, autonomy, and much more.

Section 2 opens the paper with remarks on various trends in the field of bipedal robotics. The large and very interesting literature addressing monopedal robots, polypedal robots, and planar bipedal robots is not touched here, and even the presentation of $3 \mathrm{D}$ bipedal walking is far from exhaustive. Sect. 3 overviews the method of Poincaré for establishing stability properties of periodic orbits of hybrid systems. One of the great advantages of the method of Lyapunov for analyzing the stability properties of equilibria in nonlinear systems is that one does not need to compute a solution of the model. In the case of periodic orbits, computing solutions seems to be unavoidable and hence taking advantage of invariance and time-scale properties to simplify the analysis becomes even more essential.

\footnotetext{
^ The work of J.W. Grizzle is supported by NSF grants ECS-909300, ECS-0600869. The work of C. Chevallereau is supported by ANR grants for the PHEMA project. The work of A. D. Ames is supported by NSF grant CNS-0953823. The work of R. W. Sinnet is supported by NSF through a Graduate Research Fellowship.
}

Section 4 on modeling is perhaps the most important part of the paper for a control engineer. The model of a bipedal robot differs from the model of a robotic manipulator precisely because the latter is bolted to the ground while the former is not. A foot remains flat on the ground without slipping only when the reaction forces and torques at the interface satisfy strict inequalities; in particular, the normal component of the ground reaction force must be positive, as the ground cannot "pull" against a foot as a bolt will for a manipulator; in addition, tangential forces must lie in a friction cone. These restrictions are obvious, but are nevertheless ignored in many publications. Getting the dynamic models right is crucial if a proposed control solution is to be taken seriously by the robotics community. Other interesting and challenging features of bipedal models depend on the gait that is being studied. A human gait, for example, has phases where the foot is in rotation about one of its edges. This occurs because foot-ground contact is typically initiated with a heel strike, followed by the foot rotating about the heel until the sole of the foot is flat on the ground. The end of the step is typically initiated by the foot rolling up on the toe prior to being lifted from the ground to begin the swing phase. The nature of the footground interface also determines the degree of actuation (or underactuation) of the corresponding dynamic model. A foot in rotation necessarily leads to underactuation, while if the foot is flat on the ground and all joints of the robot are independently actuated, the model is typically fully actuated. Honda's famous robot Asimo uses large feet and a flat-footed walking gait in order to avoid the control complications arising from underactuation; on the other hand, its control system is obviously meeting all of the unilateral constraints required to keep the foot flat on the ground and not slipping.

Sections 5 and 6 summarize two control design methods that are being pursued by subsets of the authors. 
The primary aim of the work in Sect. 5 is to confront the issue of underactuation. A model is studied where the foot is replaced with a point contact. This can be thought of as walking on stilts or as walking with very small feet so that foot rotation, and hence underactuation, is unavoidable. This study is important precisely because dealing with underactuation has been a stumbling block in the formal development of control laws with provable stability properties for bipedal robots. The hope is that dealing with a human-like gait, with its mix of fully actuated and underactuated phases, will then be relatively straightforward; work on planar robots reported in Choi and Grizzle [2005], Chevallereau et al. [2008] lends credence to this hope, but the question is open at this time.

The work presented in Sect. 6 is in some sense following precisely the opposite path. The primary objective has been to develop control techniques which naturally deal with the multi-phase nature of bipedal locomotion, with the early emphasis on gaits which have only fully actuated phases. Extensions to address underactuation are now being considered and are presented here. In particular, the main idea is that in the fully actuated domains the sagittal and coronal dynamics of the 3D biped can be decoupled using a variant of geometric reduction termed functional Routhian reduction. It is then only necessary to control the sagittal dynamics of the biped which is achieved through controlled symmetries - this shapes the potential energy of the system to mimic a passive biped walking down a shallow slope. Since both of these control laws require full actuation they are implemented on the fully actuated domains and "local" control laws, motivated by ideas similar to those presented in Sect. 5, are implemented on the underactuated domains to achieve the transitions through these domains.

The paper is concluded in Sect. 7 with a discussion of open problems.

\section{BRIEF COMMENTS ON THE LITERATURE}

There is a very broad spectrum of approaches to achieving stable bipedal locomotion. At one end are the highly visible humanoids, that is, robots which are inspired by human morphology. This class includes bipeds such as Honda's ASIMO, HRP-2 and Johnnie as discussed in Sakagami et al. [2002], Hirukawa et al. [2004], Kajita et al. [2004], Pfeiffer et al. [2002]. These robots represent advanced hardware development efforts focused on integrating machine vision, portable power sources, artificial intelligence, and force sensing, with attention paid to durability, packaging, etc. As such, upright, stable bipedal locomotion is only one piece of the overall effort and, largely for reasons of expediency, the designers of these robots have adopted a quasi-static notion of gait stability. The control algorithms utilized in these robots are based upon heuristics, specifically, a technique known as the zero moment point $^{1}$ (ZMP), see Goswami [1999], Vukobratović et al. [2006], which boils down to maintaining the center of pressure of the ground reaction forces on the stance foot

\footnotetext{
1 This is a widely used 'criterion' for stability in the robotics literature. It was proved in Choi and Grizzle [2005] that the ZMP condition alone is not sufficient for asymptotic stability of a periodic motion.
}

strictly within the convex hull of the foot. This results in a quasi-statically stable (flat-footed) walking gait rather than being dynamically stable as is the case for humans (where walking is sometimes characterized as "controlled falling").

At the other end of the spectrum are the minimalist bipeds where dynamic stability is achieved as much as possible through mechanical design instead of feedback control; Kuo [1999, 2002], Collins et al. [2005], Anderson et al. [2005], Wisse and van der Linde [2007] are good examples of this work. These efforts are motivated by the results of McGeer [1988, 1990], who analyzed and built planar, passive bipedal walkers, i.e., no actuation, which could walk stably down shallow slopes. Subsequently, robots with this general principle at their core have been constructed, as described in Collins et al. [2005], based on injecting small amounts of energy into passive-type bipeds. The result is very "human-looking" walking, but the remarkable elegance and economy of these walkers comes at the cost of poor ability in achieving tasks other than walking at a fixed speed; they cannot climb stairs, pause, turn or run.

Most research on dynamic robotic legged locomotion is taking place in between these two extremes. The recent book by Westervelt et al. [2007] and the review paper by Hürmüzlü et al. [2004] provide an extensive overview of the state of the art up to early 2006; further information is available in Spong and Bullo [2005], Holmes et al. [2006], Ames and Gregg [2007], Wisse and van der Linde [2007], Kuo [2007], Chevallereau et al. [2009b] and references therein.

\section{PRELIMINARIES ON HYBRID MODELS, PERIODIC SOLUTIONS, AND POINCARÉ MAPS}

This section overviews two types of hybrid models which occur frequently in models of bipedal locomotion. The dynamic models described in Sect. 4 for bipedal locomotion naturally lead to hybrid systems as given here. The section also introduces two primary tools of stability analysis for periodic orbits in such models, namely the method of Poincaré sections and the notion of a hybrid invariant manifold.

\subsection{Systems with Impulse Effects or Single-Domain Hybrid Models}

To define a $C^{1}$ system with impulse effects, consider

$$
\dot{x}=f(x),
$$

where the state manifold $\mathcal{X}$ is an open connected subset of $\mathbb{R}^{n}$, and $f$ is a $C^{1}$ vector field on $\mathcal{X}$. A switching surface $\mathcal{S}$ is a co-dimension one $C^{1}$ submanifold with $\mathcal{S}=\{x \in$ $\left.\mathcal{X} \mid H(x)=0, \dot{H}(x)=L_{f} H(x)<0\right\}$, where $H: \mathcal{X} \rightarrow \mathbb{R}$ is $C^{1}$ and $\mathcal{S} \neq \emptyset$; because $\forall x \in \mathcal{S}, L_{f} H(x)<0$, it follows that $\frac{\partial H}{\partial x}(x) \neq 0$. A transition or reset $^{2}$ map is a $C^{1}$ function $\Delta: \mathcal{S} \rightarrow \mathcal{X}$, where $\mathcal{S} \cap \Delta(\mathcal{S})=\emptyset$, that is, the image of the reset map is disjoint from its domain. A $C^{1}$ autonomous system with impulse effects is written as

\footnotetext{
2 When the reset map corresponds to the swing leg impacting the ground, it is commonly called an impact map instead of a reset map.
} 


$$
\bar{\Sigma}:\left\{\begin{array}{cl}
\dot{x}=f(x) & x^{-} \notin \mathcal{S} \\
x^{+}=\Delta\left(x^{-}\right) & x^{-} \in \mathcal{S},
\end{array}\right.
$$

where $x^{-}(t)=\lim _{\tau \nearrow t} x(\tau)$ and $x^{+}(t)=\lim _{\tau \searrow t} x(\tau)$ are the left and right limits of a trajectory, $x(t)$. For compactness of notation, an autonomous system with impulse effects (2) will sometimes be denoted as a 4-tuple, $\bar{\Sigma}=(\mathcal{X}, \mathcal{S}, \Delta, f)$.

In simple terms, a solution of (2) is specified by the differential equation (1) until its state "impacts" the hyper surface $\mathcal{S}$ at some time $t_{I}$. At $t_{I}$, the reset map $\Delta$ compresses the impact event into an instantaneous moment of time, resulting in a discontinuity in the state trajectory. The reset map provides the new initial condition from which the differential equation evolves until the next impact with $\mathcal{S}$. In order to avoid the state having to take on two values at the "impact time" $t_{I}$, the impact event is, roughly speaking, described in terms of the values of the state "just prior to impact" at time " $t_{I}^{-}$", and "just after impact" at time " $t_{I}^{+}$". These values are represented by $x^{-}$and $x^{+}$, respectively. A formal definition of a solution can be written down by piecing together appropriately initialized solutions of (1). A choice must be made whether the solution is a left- or a right-continuous function of time at each impact event; here, solutions are assumed to be right continuous. Other useful notions of a solution can be found in Filippov [1960], Ye et al. [1998], Haddad et al. [2006], Goebel et al. [2009], Lygeros et al. [2003]. Because we are interested in the local stability properties of periodic orbits, we will exclude Zeno and other complex behavior from the systems under study; see Or and Ames [2008, 2009], Lamperski and Ames [2008], Goebel et al. [2009].

\subsection{Periodic Orbits and the Poincaré Return Map for Single-Domain Models}

Cyclic behaviors such as walking are represented as periodic orbits of systems with impulse effects. A solution $\varphi\left(t, t_{0}, x_{0}\right)$ of an autonomous system $\bar{\Sigma}$ is periodic if there exists a finite $T>0$ such that $\varphi\left(t+T, t_{0}, x_{0}\right)=\varphi\left(t, t_{0}, x_{0}\right)$ for all $t \in\left[t_{0}, \infty\right)$. A set $\mathcal{O} \subset \mathcal{X}$ is a periodic orbit if $\mathcal{O}=\left\{\varphi\left(t, t_{0}, x_{0}\right) \mid t \geq t_{0}\right\}$ for some periodic solution $\varphi\left(t, t_{0}, x_{0}\right)$. If a periodic solution has an impact event, then the corresponding periodic orbit $\mathcal{O}$ is not closed; see Grizzle et al. [2001], Morris and Grizzle [2005]. Let $\overrightarrow{\mathcal{O}}$ denote its set closure. Notions of stability in the sense of Lyapunov, asymptotic stability, and exponential stability of orbits follow the standard definitions of orbital stability as in [Khalil, 1996, pp. 302], Grizzle et al. [2001], Nersesov et al. [2002].

The method of Poincaré sections is widely used to determine the existence and stability of periodic orbits in a broad range of system models, such as time-invariant and periodically-time-varying ordinary differential equations Parker and Chua [1989], Guckenheimer and Holmes [1996], hybrid systems consisting of several time-invariant ordinary differential equations linked by event-based switching mechanisms and re-initialization rules Grizzle et al. [2001], Nersesov et al. [2002], Roup et al. [2003], differentialalgebraic equations Hiskens [2001], and relay systems with hysteresis Goncalves et al. [2001], to name just a few. The analytical details may vary significantly from one class of

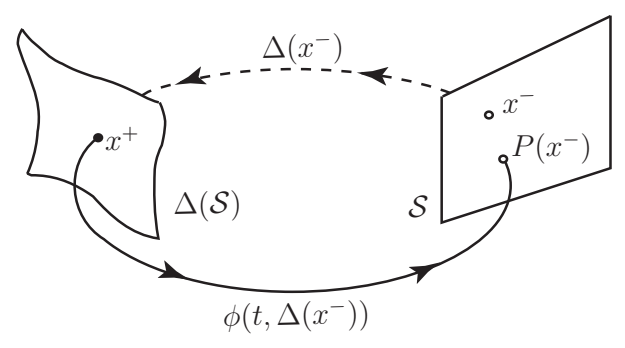

Fig. 1. Geometric interpretation of a Poincaré return map $P: \mathcal{S} \rightarrow \mathcal{S}$ for a system with impulse effects. The Poincaré section is selected as the switching surface, $\mathcal{S}$. A periodic orbit exists when $P\left(x^{-}\right)=x^{-}$. If solutions are assumed to be right continuous, then $x^{-}$is not an element of the orbit; with left-continuous solutions, $\Delta\left(x^{-}\right)$is not an element of the orbit.

models to another; for example, the trivial eigenvalues of Poincaré maps for hybrid systems are zero while the trivial eigenvalues of Poincaré maps for smooth dynamical systems are one (see Wendel and Ames [2010]). Yet, despite these sometimes subtle differences, on a conceptual level the method of Poincaré is consistent and straightforward: sample the solution of a system according to an eventbased or time-based rule and then evaluate the stability properties of equilibrium points (also called fixed points) of the sampled system. The sampled values give rise to the Poincaré return map; see Fig. 1. Fixed points of the Poincaré map correspond to periodic orbits (limit cycles) of the underlying system.

When using the method of Poincaré to study systems with impulse effects, it is natural to select $\mathcal{S}$ as the Poincaré section. To define the return map, let $\phi\left(t, x_{0}\right)$ be the maximal solution of (1) with initial condition $x_{0}$ at time $t_{0}=0$. The time-to-impact function, $T_{I}: \mathcal{X} \rightarrow \mathbb{R}$, is the time from initialization to the first intersection with the set $\mathcal{S}$ and is in general a partial map

$$
\begin{aligned}
& T_{I}\left(x_{0}\right):=\inf \left\{t \geq 0 \mid \phi\left(t, x_{0}\right) \in S\right\} \text { if } \exists t \text { such that } \\
& \phi\left(t, x_{0}\right) \in \mathcal{S} \text {. }
\end{aligned}
$$

The Poincaré return map, $P: \mathcal{S} \rightarrow \mathcal{S}$, is then the partial map

$$
P(x)=\phi\left(T_{I} \circ \Delta(x), \Delta(x)\right) .
$$

A periodic orbit $\mathcal{O}$ is period-one if its closure intersects $\mathcal{S}$ at exactly one point, $x^{*}=\overline{\mathcal{O}} \cap \mathcal{S}$; because $x^{*}=P\left(x^{*}\right)$, it is called a fixed point. A period-one orbit is transversal to $\mathcal{S}$ if $L_{f} H\left(x^{*}\right)=\frac{\partial H}{\partial x}\left(x^{*}\right) f\left(x^{*}\right) \neq 0$ (in words, the vector field $f$ is not tangent to $\mathcal{S}$ at the point $\left.x^{*}\right)$. For convenience, define the partial function $\phi_{T_{I}}(x)=\phi\left(T_{I}(x), x\right)$ so that the Poincaré return map can be written as

$$
P(x)=\phi_{T_{I}} \circ \Delta(x) .
$$

For the case of autonomous systems with impulse effects, the method of Poincaré sections is formalized in the following theorem.

Theorem 1. (Morris and Grizzle [2009], Method of Poincaré Sections) If the $C^{1}$ autonomous system with impulse effects $\bar{\Sigma}=(\mathcal{X}, \mathcal{S}, \Delta, f)$ has a periodic orbit $\mathcal{O}$ that is transversal to $\mathcal{S}$, then the following are equivalent:

i) $x^{*}$ is an exponentially stable (respectively, asymptotically stable, or stable in the sense of Lyapunov) fixed point of $P$; 
ii) $\mathcal{O}$ is an exponentially stable (respectively, asymptotically stable, or stable in the sense of Lyapunov) periodic orbit.

Remark 2. Results in [Westervelt et al., 2007, Sect. 4.2.2] show that if $\mathcal{O}$ is transversal to $\mathcal{S}$ and $\Sigma=(\mathcal{X}, \mathcal{S}, \Delta, f)$ is $C^{1}$, then the partial map $P$ is well-defined and differentiable at a fixed point $x^{*}$, and hence exponential stability can be checked by evaluating eigenvalues of the Jacobian of $P$ at $x^{*}$.

\subsection{Multi-domain Hybrid Models}

This section will address systems with $N_{p} \geq 2$ continuous domains and discrete transitions between the domains. Such models frequently occur in bipedal locomotion. We will assume phases are executed in a fixed order $1 \rightarrow 2 \rightarrow$ $\cdots \rightarrow N_{p} \rightarrow 1$. We will use the notation $N_{p}+1=1$ (addition modulo $N_{p}$ ).

For each $1 \leq i \leq N_{p}$, let $\mathcal{X}_{\mathrm{i}}$ be an open connected subset of $\mathbb{R}^{n_{\mathrm{i}}}$ upon which is defined an autonomous differential equation $\mathcal{F}_{\mathrm{i}}$. Let $\mathcal{S}_{\mathrm{i}}^{\mathrm{i}+1}$ be an embedded submanifold of codimension one in the state space $\mathcal{X}_{\mathrm{i}}$ that determines when a transition from $\mathcal{X}_{\mathrm{i}}$ to $\mathcal{X}_{\mathrm{i}+1}$ takes place according to the reset map $\Delta_{\mathrm{i}}^{\mathrm{i}+1}: \mathcal{S}_{\mathrm{i}}^{\mathrm{i}+1} \rightarrow \mathcal{X}_{\mathrm{i}+1}$.

The corresponding hybrid model is written using the notation in Guckenheimer and Johnson [1995] as

$$
\bar{\Sigma}:\left\{\begin{array}{c}
\mathcal{X}=\left\{\mathcal{X}_{\mathrm{i}}\right\}_{\mathrm{i}=1}^{N_{p}}: \mathcal{X}_{\mathrm{i}} \subset \mathbb{R}^{n_{\mathrm{i}}} \\
\mathcal{F}=\left\{f_{\mathrm{i}}\right\}_{\mathrm{i}=1}^{N_{p}}: \dot{x}_{\mathrm{i}}=f_{\mathrm{i}}\left(x_{\mathrm{i}}\right) \\
\mathcal{S}=\left\{\mathcal{S}_{\mathrm{i}}^{\mathrm{i}+1}\right\}_{\mathrm{i}=1}^{N_{p}}: \mathcal{S}_{\mathrm{i}}^{\mathrm{i}+1}=\left\{x_{\mathrm{i}} \in \mathcal{X}_{\mathrm{i}} \mid H_{\mathrm{i}}^{\mathrm{i}+1}\left(x_{\mathrm{i}}\right)=0,\right. \\
\left.\dot{H}_{\mathrm{i}}^{\mathrm{i}+1}\left(x_{\mathrm{i}}\right)<0\right\} \\
\Delta=\left\{\Delta_{\mathrm{i}}^{\mathrm{i}+1}\right\}_{\mathrm{i}=1}^{N_{p}}: x_{\mathrm{i}+1}^{+}=\Delta_{\mathrm{i}}^{\mathrm{i}+1}\left(x_{\mathrm{i}}^{-}\right) .
\end{array}\right.
$$

Under assumptions analogous to those for the singledomain model, a unique, maximal solution of the multidomain model can be constructed by piecing together trajectories of the flows $\mathcal{F}_{\mathrm{i}}$ in such a way that a transition occurs when a flow intersects a switching hyper-surface, $\mathcal{S}_{i}^{\mathrm{i}+1}$, and at each transition, the new initial condition is determined by the reset maps $\Delta_{i}^{\mathrm{i}+1}$. To avoid chattering, it is assumed that $\Delta_{\mathrm{i}}^{\mathrm{i}+1}\left(\mathcal{S}_{\mathrm{i}}^{\mathrm{i}+1}\right) \cap \mathcal{S}_{\mathrm{i}+1}^{\mathrm{i}+2}=\emptyset$, so that a solution through a domain must have a non-zero duration.

Remark 3. Note that the hybrid model $\bar{\Sigma}$ introduced in (4) is equivalent to the definition of a hybrid system on a cycle (as studied in Lamperski and Ames [2008]), which is typically stated as a tuple,

$$
\mathscr{H}=\bar{\Sigma}=(\Gamma, \mathcal{X}, \mathcal{S}, \Delta, \mathcal{F}),
$$

where $\Gamma$ is the directed graph with vertices $\left\{1,2, \ldots, N_{p}\right\}$ and edges connecting vertex $i$ to vertex $i+1$.

\subsection{Periodic Orbits and the Poincaré Return Map for Multi-Domain Models}

Let $\mathcal{X}=\mathcal{X}_{1} \cup \mathcal{X}_{2} \cdots \cup \mathcal{X}_{N_{p}}$. A solution $\phi(t)$ of (4) is periodic if there exists a finite $T>0$ such that $\phi(t+$ $T)=\phi(t)$ for all $t \in\left[t_{0}, \infty\right)$. A set $\mathcal{O} \subset \mathcal{X}$ is a periodic orbit of (4) if $\mathcal{O}=\left\{\phi(t) \mid t \geq t_{0}\right\}$ for some periodic solution $\phi(t)$. The definitions of orbital stability in the sense of Lyapunov, orbital asymptotic stability, and orbital exponential stability are analogous to those for systems with impulse effects. A periodic orbit $\mathcal{O}$ is transversal to $\mathcal{S}_{\mathrm{i}}^{\mathrm{i}+1}$ if its closure intersects $\mathcal{S}_{\mathrm{i}}^{\mathrm{i}+1}$ in exactly one point, and for $x_{\mathrm{i}}^{*}:=\overline{\mathcal{O}} \cap \mathcal{S}_{\mathrm{i}}^{\mathrm{i}+1}, L_{f_{\mathrm{i}}} H_{\mathrm{i}}^{\mathrm{i}+1}\left(x_{\mathrm{i}}^{*}\right):=\frac{\partial H_{\mathrm{i}}^{\mathrm{i}+1}}{\partial x_{\mathrm{i}}}\left(x_{\mathrm{i}}^{*}\right) f_{\mathrm{i}}\left(x_{\mathrm{i}}^{*}\right) \neq 0$. A periodic orbit $\mathcal{O}$ is transversal if it is transversal to $\mathcal{S}_{\mathrm{i}}^{\mathrm{i}+1}$ for all $i$. In the case of a bipedal robot, a nontrivial, transversal, periodic orbit will also be referred to as periodic locomotion.

The Poincaré return map remains the mathematical tool of choice for determining the existence and stability properties of periodic orbits. As in (3), define the phase- $i$ timeto-impact function, $T_{I, \mathrm{i}}: \mathcal{X}_{\mathrm{i}} \rightarrow \mathbb{R}$ as the partial map

$$
\begin{array}{r}
T_{I, \mathrm{i}}\left(x_{0}\right):=\inf \left\{t \geq 0 \mid \phi_{\mathrm{i}}\left(t, x_{0}\right) \in \mathcal{S}_{\mathrm{i}}^{\mathrm{i}+1}\right\} \quad \text { if } \exists t \text { such that } \\
\phi_{\mathrm{i}}\left(t, x_{0}\right) \in \mathcal{S}_{\mathrm{i}}^{\mathrm{i}+1},
\end{array}
$$

where $\phi_{\mathrm{i}}\left(t, x_{0}\right)$ is an integral curve of (4) corresponding to $\phi_{\mathrm{i}}\left(0, x_{0}\right)=x_{0}$. The generalized Poincaré phase- $i$ map $P_{\mathrm{i}}: \mathcal{S}_{\mathrm{i}-1}^{\mathrm{i}} \rightarrow \mathcal{S}_{\mathrm{i}}^{\mathrm{i}+1}$ is the partial map

$$
P_{\mathrm{i}}\left(x_{\mathrm{i}-1}\right):=\phi_{\mathrm{i}}\left(T_{I, \mathrm{i}}\left(\Delta_{\mathrm{i}-1}^{\mathrm{i}}\left(x_{\mathrm{i}-1}\right)\right), \Delta_{\mathrm{i}-1}^{\mathrm{i}}\left(x_{\mathrm{i}-1}\right)\right) .
$$

The Poincaré return map can be defined as the composition of the generalized Poincaré phase- $i$ maps, starting at any point in the cycle $1 \rightarrow 2 \rightarrow \cdots \rightarrow N_{p} \rightarrow 1$. Here, for convenience, we start it at $i=1$, so that

$$
P:=P_{N_{p}} \circ \cdots \circ P_{1} \text {. }
$$

Theorem 4. (Connecting Multi-Phase Models to Single-Phase Models) Let $P$ be the Poincaré return map defined in (7) for the multi-phase model in (4). $P$ is also the Poincaré return map for the system with impulse effects (2), where $\mathcal{X}=\mathcal{X}_{1}, f=f_{1}, \mathcal{S}:=\mathcal{S}_{1}^{2}$ and $\Delta:=\Delta_{N_{p}}^{1} \circ$ $P_{N_{p}} \circ \cdots \circ P_{2}$.

Proof. This follows immediately from the construction of the Poincaré return maps in (4) and (7).

Remark 5. It is emphasized that this observation is important because it allows results developed for single-domain models of the form (2) to be applied to models with multiple phases, as in (4). In particular, suppose that the multi-domain hybrid model (4) is $C^{1}$ in each phase and has a transversal periodic orbit $\mathcal{O}$. Then, results in [Westervelt et al., 2007, Sect. 4.2.2] show that $\Delta:=\Delta_{N_{p}}^{1} \circ P_{N_{p}} \circ \cdots \circ P_{2}$ is $C^{1}$ in a neighborhood of $x^{*}=\mathcal{O} \cap \mathcal{S}$, and thus $P$ is $C^{1}$ in a neighborhood of $x^{*}$. Exponential stability can therefore be checked by evaluating eigenvalues of the Jacobian of $P$ at $x^{*}$.

\subsection{Determining Orbital Stability on the Basis of a Restriction Dynamics}

This section identifies properties of the autonomous hybrid system (2) under which the exponential stability of a periodic orbit can be determined on the basis of a hybrid restriction dynamics. The key hypothesis will be the existence of an embedded submanifold that is invariant under both the continuous and discrete portions of the hybrid model (2). The design of static and dynamic state variable feedbacks that create invariant submanifolds for systems modeled by ordinary differential equations is a well-studied problem and plays a prominent role in the 
notion of the zero dynamics. How to design feedbacks that achieve invariance under the reset map is treated in Sect. 5.3 .

The following definitions formalize notions of hybrid invariance and restriction dynamics.

Definition 6. For an autonomous system with impulse effects $\bar{\Sigma}=(\mathcal{X}, \mathcal{S}, \Delta, f)$, a submanifold $Z \subset \mathcal{X}$ is forward invariant if for each point $x$ in $\mathcal{Z}, f(x) \in T_{x} Z$ where $T_{x} Z$ is the tangent space of the manifold $Z$ at the point $x$. A submanifold $Z$ is impact invariant in an autonomous system with impulse effects $\bar{\Sigma}$, if for each point $x$ in $\mathcal{S} \cap Z$, $\Delta(x) \in Z$. A submanifold $Z$ is hybrid invariant if it is both forward invariant and impact invariant.

Definition \%. If a $C^{1}$ embedded submanifold $Z$ is hybrid invariant and $\mathcal{S} \cap Z$ is $C^{1}$ with dimension one less than that of $Z$, then

$$
\left.\bar{\Sigma}\right|_{Z}:\left\{\begin{array}{clrl}
\dot{z} & =\left.f\right|_{Z}(z) & z^{-} \notin \mathcal{S} \cap Z \\
z^{+}=\left.\Delta\right|_{\mathcal{S} \cap Z}\left(z^{-}\right) & z^{-} \in \mathcal{S} \cap Z,
\end{array}\right.
$$

is called a hybrid restriction dynamics of the autonomous system $\bar{\Sigma}$, where $\left.f\right|_{Z}$ and $\left.\Delta\right|_{\mathcal{S} \cap Z}$ are the restrictions of $f$ and $\Delta$ to $Z$ and $\mathcal{S} \cap Z$, respectively. The hybrid restriction

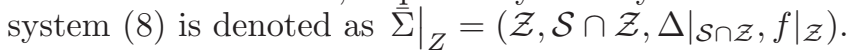

If a system $\bar{\Sigma}$ has a periodic orbit $\mathcal{O}$ lying in a hybrid invariant manifold $Z$, then $\mathcal{O}$ is a periodic orbit of the resulting hybrid restriction dynamics. In this case hybrid invariance of $\mathcal{Z}$ is reflected in the Poincaré map as

$$
P(\mathcal{S} \cap Z) \subset \mathcal{S} \cap Z \text {. }
$$

On the basis of (9), the restricted Poincaré map,

$$
\rho: \mathcal{S} \cap Z \rightarrow \mathcal{S} \cap Z,
$$

is defined as $\rho=\left.P\right|_{Z}$.

The following result shows that if the invariant manifold is sufficiently rapidly attractive, then stability of the periodic orbit in the complete model can be deduced on the basis of the restricted Poincaré map.

Theorem 8. (Morris and Grizzle [2009], Reduced Dimensional Stability Test) Consider a family of $C^{1}$ autonomous systems with impulse effects with the vector field of each member depending on a real parameter $\epsilon>0$, $\bar{\Sigma}^{\epsilon}=\left(\mathcal{X}, \mathcal{S}, \Delta, f^{\epsilon}\right)$. Let $Z$ be a $k$-dimensional $C^{1}$ embedded submanifold of $\mathcal{X}$, with $k \geq 1$. Suppose in addition that:

(a) $\mathcal{S} \cap Z$ is a $C^{1},(k-1)$-dimensional embedded submanifold of $\mathcal{S}$;

(b) $f^{\epsilon}$ restricted to $\mathcal{Z}$ is independent of $\epsilon$, so that $\left.f\right|_{Z}=$ $\left.f^{\epsilon}\right|_{Z}$ for any $\epsilon \in(0, \infty)$

(c) $Z$ is hybrid invariant;

(d) there exists a periodic orbit $\mathcal{O}$ of $\bar{\Sigma}^{\epsilon}=\left(\mathcal{X}, \mathcal{S}, \Delta, f^{\epsilon}\right)$ that is contained in $Z$ and with the corresponding fixed-point denoted by $x^{*}$; and

(e) there exists a function $K:(0, \infty) \rightarrow[0, \infty)$ such that $\lim _{\epsilon \backslash 0} K(\epsilon)=0$, and $\forall \epsilon>0, \exists \delta>0$ such that ${ }^{3} \forall x_{0} \in B_{\delta}\left(\Delta\left(x^{*}\right)\right)$, dist $\left(\phi_{T_{I}^{\epsilon}}^{\epsilon}\left(x_{0}\right), Z\right) \leq$ $K(\epsilon) \operatorname{dist}\left(x_{0}, Z\right)$.

Then the restriction dynamics $\left.\bar{\Sigma}^{\epsilon}\right|_{Z}$ and fixed point are both independent of $\epsilon$. In addition, there exists $\bar{\epsilon}>0$ such that for $0<\epsilon<\bar{\epsilon}$, the following are equivalent:

\footnotetext{
$3 B_{r}(x)$ denoted the open ball of radius $r$ about the point $x$.
}

i) $x^{*}$ is an exponentially stable fixed point of $P^{\epsilon}$, and

ii) $x^{*}$ is an exponentially stable fixed point of $\rho$,

where $P^{\epsilon}=\phi_{T_{I}^{\epsilon}}^{\epsilon} \circ \Delta$ and $\rho=\left.P^{\epsilon}\right|_{\mathcal{Z}}$.

\section{DYNAMIC MODELS}

Robotic legged locomotion is characterized by the fact that the contact between the robot and its environment (the ground) is unilateral and intermittent. These characteristics entail specific challenges for the control of bipedal walking. A walking gait can be decomposed into distinct phases, with the dynamic model in each phase depending on the nature of the contact between the feet and the ground. Since the ground cannot pull on the foot, unilateral and other constraints exist on the forces and moments exerted by the ground on the foot. If these constraints are satisfied, the robot evolves according to the dynamics of the given phase, and when the constraints are violated, the phase changes and so must the model. Thus the natural way to describe a walking motion is in the form of a hybrid system.

\subsection{Generalities}

The robot itself is classically modeled as a tree structure composed of rigid links. When contact occurs between the feet and the ground, it is assumed to be a rigid contact. With these assumptions, one way to obtain a model for the various phases of a walking gait is to first construct a Lagrangian model of the robot in general position (i.e., no assumptions on ground contact), and then analyze various ground contact conditions by imposing holonomic constraints on the model and computing the corresponding contact forces and moments. To begin this modeling approach, let $R_{0}$ be a fixed inertial (or world) frame and let $R_{b}$ be a reference frame attached to some point on the robot, as in Fig. 2. Let $p_{b} \in \mathbb{R}^{3}$ be the Cartesian position of $R_{b}$ with respect to $R_{0}$ and let $\phi_{b} \in$ $S O(3)$ be the orientation. Where convenient, we identify an open subset of $S O(3)$ with an open subset of $\mathbb{R}^{3}$ using Euler angles ${ }^{4}$. Next, let $q \in \mathcal{Q}$ be an $N$-dimensional vector of body (or shape) coordinates ${ }^{5}$ for the robot. Then $q_{e}=\left(p_{b}^{\prime}, \phi_{b}^{\prime}, q\right)^{\prime} \in \mathcal{Q}_{e}=\mathbb{R}^{3} \times S O(3) \times Q$ is a set of generalized coordinates for the robot.

Following standard techniques, the robot's Lagrangian is computed as a functional acting on $T \mathcal{Q}_{e}$. The Lagrangian is defined to be the difference between the kinetic and potential energies

$$
\mathcal{L}_{e}\left(q_{e}, \dot{q}_{e}\right):=K_{e}\left(q_{e}, \dot{q}_{e}\right)-V_{e}\left(q_{e}\right) .
$$

From Hamilton's principle, the equations of motion can be calculated directly from the Lagrangian as

$$
\frac{d}{d t} \frac{\partial \mathcal{L}_{e}}{\partial \dot{q}_{e}}-\frac{\partial \mathcal{L}_{e}}{\partial q_{e}}=\Gamma\left(q_{e}, \dot{q}_{e}\right)+B_{e}\left(q_{e}\right) u
$$

\footnotetext{
4 While singular configurations exist in this identification, they are not relevant for upright configurations of the torso that occur in normal walking.

5 These are coordinates tied to the reference frame $R_{b}$ on the body of the robot. If the robot consists of rigid links connected through onedimensional revolute joints, then the relative joint angles constitute a set of body coordinates.
} 


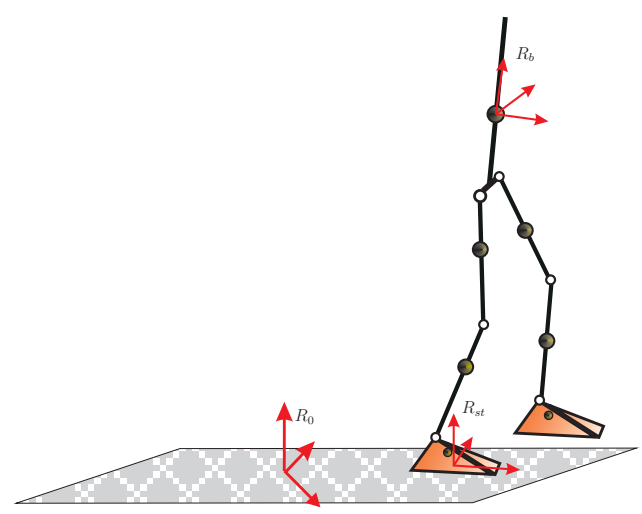

Fig. 2. A frame $R_{b}$ is attached to the body. The position and orientation of the robot are expressed with respect to a fixed inertial frame $R_{0}$. A frame $R_{s t}$ attached to the stance foot is useful for expressing the contact conditions.

where $B_{e}(q) u$ is the vector of actuator torques and $\Gamma\left(q_{e}, \dot{q}_{e}\right)$ represents other nonconservative forces (such as joint friction) [Goldstein et al., 2002, pp. 34-45]. The effect of gravity is accounted for in the potential energy. The torque distribution matrix $B_{e}(q)$ depends only on the body coordinates; its columns are assumed involutive and (point-wise) linearly independent so that in appropriate coordinates the matrix is constant, with rank equal to the number of actuators, $N_{a}$. The kinetic energy is a quadratic, positive definite function of the generalized velocities, and hence (12) leads to the standard robot equations

$$
D_{e}\left(q_{e}\right) \ddot{q}_{e}+C_{e}\left(q_{e}, \dot{q}_{e}\right) \dot{q}_{e}+G_{e}\left(q_{e}\right)-\Gamma\left(q_{e}, \dot{q}_{e}\right)=B_{e}(q) u \text {. }
$$

From here on out, for simplicity, the term $\Gamma\left(q_{e}, \dot{q}_{e}\right)$ will be dropped; it is very easy to add it back in when required. $D_{e}\left(q_{e}\right)$ is the $(N+6) \times(N+6)$ inertia matrix, $C_{e}\left(q_{e}, \dot{q}_{e}\right) \dot{q}_{e}$ is the vector of Coriolis and centrifugal effects, $G_{e}\left(q_{e}\right)$ is the gravitational force, $u$ is the vector of inputs (i.e, the actuator torques). Sometimes, to simplify notation, the Coriolis, centrifugal and gravity terms are grouped into a single vector $H_{e}\left(q_{e}, \dot{q}_{e}\right)=C_{e}\left(q_{e}, \dot{q}_{e}\right) \dot{q}_{e}+G_{e}\left(q_{e}\right)$.

In walking, at least one foot is in contact with the ground. In this presentation, we will consider two types of contact, namely, the foot is either flat on the ground, or the contact is along an edge of the foot, either the toe or the heel, leading to rotation about the corresponding axis. The term single support (SS) means that one foot is in contact with the ground, flat or otherwise, and double support (DS) means that both feet are in contact with the ground. To be clear, the model (13) assumes "no support". The models corresponding to various types of ground contact can be obtained by imposing holonomic and kinematic constraints on $(13)$.

A holonomic constraint $\eta\left(q_{e}\right)$ is a multidimensional vector with each element corresponding to a function of the generalized position coordinates that must be satisfied in order to achieve the appropriate behavior on a given phase of a walking gait. For example, some models of bipedal walking assume the stance knee is locked in certain parts of the gait; a more ubiquitous example is the stance foot must be constrained to the ground. Given a generic set of holonomic constraints, $\eta: \mathcal{Q}_{e} \rightarrow \mathbb{R}^{c}$, the dynamic model which imposes them can be written as

$$
D_{e}\left(q_{e}\right) \ddot{q}_{e}+H_{e}\left(q_{e}, \dot{q}_{e}\right)+J\left(q_{e}\right)^{\prime} \lambda=B_{e}(q) u,
$$

where $\lambda$ is commonly referred to as a Lagrange multiplier and $J\left(q_{e}\right)=\frac{\partial \eta\left(q_{e}\right)}{\partial q_{e}}$ is the Jacobian of the constraints. Setting the second derivative of the holonomic constraint to zero

$$
J\left(q_{e}\right) \ddot{q}_{e}+\frac{\partial}{\partial q_{e}}\left[J\left(q_{e}\right) \dot{q}_{e}\right] \dot{q}_{e}=0
$$

completes the dynamic model, that is, defines $\lambda$ and $\ddot{q}_{e}$, as long as the holonomic constraint has full rank.

The physical meaning of the Lagrange multiplier $\lambda$ depends on how the holonomic constraint is expressed. In the case of locking a single degree of freedom representation of a knee, for example, it is straightforward to see that $\lambda$ is the torque required about the axis of the joint in order to maintain a constant position. On the other hand, holonomic constraints arising from the forces exerted by the ground on a foot act about multiple axes, and interpreting them is very difficult unless they are expressed in the correct reference frame.

Let $R_{s t}$ be a reference frame attached to the stance foot as in Fig. 2. The position and orientation of the frame $R_{s t}$ in the inertial frame $R_{0}$ can be expressed by a $(4 \times 4)$ transformation matrix denoted $T_{0}^{s t}$, which is a function of $q_{e}$. The linear and angular velocities of the foot, $v_{s t}$ and $\omega_{s t}$, are then defined by

$$
\left[\begin{array}{cccc}
0 & -\omega_{s t}^{z} & \omega_{s t}^{y} & v_{s t}^{x} \\
\omega_{s t}^{z} & 0 & -\omega_{s t}^{x} & v_{s t}^{y} \\
-\omega_{s t}^{y} & \omega_{s t}^{x} & 0 & v_{s t}^{z} \\
0 & 0 & 0 & 0
\end{array}\right]=\left(T_{0}^{s t}\left(q_{e}\right)\right)^{-1} \dot{T}_{0}^{s t}\left(q_{e}, \dot{q}_{e}\right)
$$

The ground contact forces can be grouped into a wrench $F_{s t}$ containing both forces and moments expressed in the frame $R_{s t}$. According to the principle of virtual work found in Dombre and Khalil [2002], Murray et al. [1993], Spong et al. [2005], the contact wrench is taken into account in the dynamic model as

$$
D_{e}\left(q_{e}\right) \ddot{q}_{e}+H_{e}\left(q_{e}, \dot{q}_{e}\right)=B_{e}(q) u+J_{s t}\left(q_{e}\right)^{\prime} F_{s t},
$$

where $J_{s t}\left(q_{e}\right)$ is full rank and satisfies

$$
\left[\begin{array}{c}
v_{s t} \\
\omega_{s t}
\end{array}\right]=J_{s t}\left(q_{e}\right) \dot{q}_{e} .
$$

The comparison of models (14) and (17) yields the relation between $\lambda$ and $F_{s t}$. Depending on the ground contact assumptions, the number of nonzero components of $F_{s t}$ varies depending on the kinematic constraint arising from setting appropriate rows of (18) to zero. We now consider different cases of foot-ground contact and detail the corresponding constraint equations along with the limits on the ground reaction forces and moments.

\subsection{Single support with flat foot contact}

The position and orientation of the stance foot is assumed to be fixed (no slipping, etc.). We will impose this with a holonomic constraint. Attach a reference frame $R_{s t}$ to the stance foot as in Fig. 2, and let ${ }^{6}$

$$
\eta_{s t}\left(q_{e}\right)=\left[\begin{array}{l}
p_{s t}\left(q_{e}\right) \\
\phi_{s t}\left(q_{e}\right)
\end{array}\right]
$$

6 Euler angles can be used for example to express the orientation. 
be a holonomic constraint containing the position and orientation of $R_{s t}$ with respect to $R_{0}$, which can be expressed in terms of the generalized coordinates $q_{e}$. It can be shown that the Jacobian $\frac{\partial \eta_{s t}}{\partial q_{e}}$ has full rank. We can write the holonomic constraint as

$$
\eta_{s t}\left(q_{e}\right)=\left[\begin{array}{c}
p_{s t} \\
\phi_{s t}
\end{array}\right]=\text { constant. }
$$

A rigid, flat contact is assumed between one foot and the ground (i.e., the stance foot), with the other foot raised above the ground (i.e., the swing foot). The ground contact forces and torques on the sole of the feet are expressed in terms of the wrench $F_{s t}$ computed in the frame $R_{s t}$. This wrench has six components,

$$
F_{s t}=\left(F_{s t}^{f x}, F_{s t}^{f y}, F_{s t}^{f z}, F_{s t}^{m x}, F_{s t}^{m y}, F_{s t}^{m z}\right)^{\prime},
$$

where the first three components are the ground reaction forces and the last three are the moments (i.e., ground reaction torques).

Because the position and orientation of the foot are fixed, their velocity and acceleration are zero. The kinematic constraint is

$$
\left[\begin{array}{c}
v_{s t} \\
\omega_{s t}
\end{array}\right]=J_{s t}\left(q_{e}\right) \dot{q}_{e}=0_{6 \times 1},
$$

where the velocities are defined in (16). Differentiating (21), the constraint on acceleration is

$$
J_{s t}\left(q_{e}\right) \ddot{q}_{e}+\frac{\partial}{\partial q_{e}}\left(\frac{\partial J_{s t}\left(q_{e}\right)}{\partial q_{e}} \dot{q}_{e}\right) \dot{q}_{e}=0_{6 \times 1} .
$$

This equation in conjunction with (17) leads to a system of equations from which the contact wrench $F_{s t}\left(q_{e}, \dot{q}_{e}, u\right)$ and $\ddot{q}_{e}$ can be computed. It follows that $F_{s t}$ is affine in the actuator torques $u$.

The models corresponding to specific phases of a gait impose specific bounds on $F_{s t}$. In order to avoid take-off (i.e., the foot lifting from the ground), the constraint is

$$
F_{s t}^{f z}>0 .
$$

To avoid linear slipping ${ }^{7}$, the constraint is

$$
\sqrt{\left(F_{s t}^{f x}\right)^{2}+\left(F_{s t}^{f y}\right)^{2}}<\mu F_{s t}^{f z}
$$

where $\mu$ is the assumed friction parameter. To use linear constraints, the friction cone (24) can be replaced by a friction pyramid, which gives

$$
\begin{aligned}
& \left|F_{s t}^{f x}\right|<\frac{\mu}{\sqrt{2}} F_{s t}^{f z}, \\
& \left|F_{s t}^{f y}\right|<\frac{\mu}{\sqrt{2}} F_{s t}^{f z} .
\end{aligned}
$$

Even if the foot is pressing on the ground and not sliding, it could be in rotation about one of its edges. Indeed, due to the finite size of the feet, and due to the unilateral nature of the contact, Vukobratović et al. [1990] and Chevallereau et al. [2009a] show that the moment produced by the ground is limited by

$$
\begin{aligned}
-l_{b} F_{s t}^{f z} & <F_{s t}^{m x}<l_{a} F_{s t}^{f z} \\
-L_{a} F_{s t}^{f z} & <F_{s t}^{m y}<L_{b} F_{s t}^{f z},
\end{aligned}
$$

\footnotetext{
7 A condition also exists also on $F_{s t}^{m z}$, but it involves the size of the foot, the friction parameter and the unknown distribution of the forces along the sole of the foot. Consequently, when modeling straight displacement, a constraint on $F_{s t}^{m z}$ in order to avoid rotational slipping is usually neglected.
}

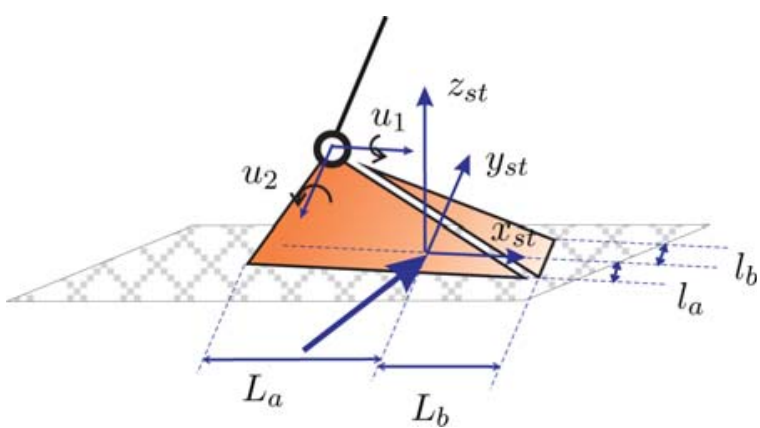

Fig. 3. The ground exerts a wrench on the foot, that is, a pure force and a moment (or torque). The wrench is expressed in the reference frame $R_{s t}$, which has been attached to an arbitrary point on the foot.

where $L_{a}, L_{b}, l_{a}, l_{b}$ are defined by the geometry of the foot as in Fig. 3. If one of the conditions in (26) is not satisfied, the foot will rotate along an edge. The constraint (26) is known as the Zero Moment Point (ZMP) condition. $^{8}$ Because a foot is typically less wide than long, the constraints in the frontal plane are more severe than in the sagittal ${ }^{9}$ plane.

The collection of constraints (23), (25), and (26) must all be satisfied in order that the assumption of walking with a foot flat on the ground be correct. These equations can be grouped as

$$
A_{F_{s t}}\left(q_{e}\right)^{\prime} F_{s t}\left(q_{e}, \dot{q}_{e}, u\right)>0,
$$

where it is noted that $F_{s t}$ depends not only on the states $q, \dot{q}$, but also on the actuator torques, $u$. The dynamic model is valid only if $F_{s t}$ satisfies the condition (27); otherwise, the assumed contact conditions are not valid and the phase of walking changes.

The phase of a walking gait can also change due to other conditions, for example the distance of the swing foot to the ground becoming zero. We introduce therefore a unilateral constraint, $h: \mathcal{Q}_{e} \rightarrow \mathbb{R}$, which parameterizes distance to impact. We can combine unilateral and holonomic constraints viz.

$$
H\left(q_{e}, \dot{q}_{e}, u\right)=\left[\begin{array}{c}
A_{F_{s t}}\left(q_{e}\right)^{\prime} F_{s t}\left(q_{e}, \dot{q}_{e}, u\right) \\
h\left(q_{e}\right)
\end{array}\right] .
$$

The domain of admissibility corresponds to the subset in which all of the previous constraints, both holonomic and unilateral, are satisfied,

$$
D=\left\{\left(\begin{array}{c}
q_{e} \\
\dot{q}_{e} \\
u
\end{array}\right) \in T \mathcal{Q}_{e} \times \mathbb{R}^{N_{a}} \mid H\left(q_{e}, \dot{q}_{e}, u\right)>0\right\} .
$$

If the state of the robot belongs to the domain of admissibility, then a simplified model can be used, corresponding to restricting the dynamics to the surface defined by the

\footnotetext{
8 The wrench $F_{s t}$ can be calculated at any point of the foot. There exist a point in the plane of the sole of the foot at which the corresponding wrench is reduced to a force and a moment about the vertical axis (i.e., the other two components of the moment are zero). This point is called Zero Moment Point. If this point belongs to the convex hull of the foot support area, the foot does not rotate. 9 The sagittal plane divides the body into left and right halves; planar robots typically evolve in the sagittal plane. The frontal plane divides the body into front and back halves; hip sway takes place in the frontal plane. The frontal plane is also called the coronal plane.
} 
holonomic constraint (20). The dynamic model then takes the form ${ }^{10}$

$$
D(q) \ddot{q}+C(q, \dot{q}) \dot{q}+G(q)=B(q) u .
$$

If each of the joints of the robot, including the ankle, is independently actuated, then the model is fully actuated, meaning $\operatorname{dim} q=\operatorname{dim} u=\operatorname{rank}$ of $B$.

Remark 9. When employing the reduced model (30), the condition of belonging to the domain of admissibility must still be checked. This step is neglected in many publications. As a result, one does not know if the closed-loop model is implicitly assuming a foot of infinite size (so that (26) is hard to violate), a "sticky" ground model, where (23) is violated, or an unrealistically large coefficient of friction in (25). Along a trajectory of (30), the holonomic constraint (20) allows the "missing" components of $q_{e}$ and $\dot{q}_{e}$ to be computed, which in conjunction with the control input used in (30), allows the contact wrench to be evaluated.

Remark 10. The constraint (26) is the most difficult to meet and hence many control strategies are devoted to its satisfaction; see Hirai et al. [1998], Kajita et al. [2003] and references therein. The difficulty in satisfying this constraint increases as the size of the feet $\left(L_{a}, L_{b}, l_{a}, l_{b}\right)$ decreases. In Sect. 4.4, we will study the situation where all of theses values are equal to zero, corresponding to a point-foot model. A control strategy which can deal with this case should be extendable to a foot of any size.

\subsection{Rotation of the foot about the toe or the heel}

A situation where the foot is not flat on the ground is analyzed next. It is assumed that the foot is in rotation along an axis aligned with the toe or the heel and that the $y$-axis of the reference frame $R_{s t}$ has been aligned with the axis of rotation of the foot. It is assumed also that the orientation of the frame $R_{s t}$ is defined by a set of three rotations, with the third rotation being along the $y$-axis, so that the orientation matrix between $R_{0}$ and $R_{s t}$ can be written as ${ }^{0} A_{s t}=\operatorname{Rot}\left(z, \phi_{s t}^{z}\right) \operatorname{Rot}\left(x, \phi_{s t}^{x}\right) \operatorname{Rot}\left(y, \phi_{s t}^{y}\right)$. It follows that the angle $\phi_{s t}^{y}$ is free, while the variables $\phi_{s t}^{x}$ and $\phi_{s t}^{z}$ are constant. Let

$$
\eta_{s t, R}\left(q_{e}\right)=\left[\begin{array}{c}
p_{s t}\left(q_{e}\right) \\
\phi_{s t}^{x}\left(q_{e}\right) \\
\phi_{s t}^{z}\left(q_{e}\right)
\end{array}\right]
$$

be the position of $R_{s t}$ with respect to $R_{0}$ and its orientation about the $x$ and $z$ axes, expressed in terms of the generalized coordinates $q_{e}$. It can be shown that the Jacobian $\frac{\partial \eta_{s t, R}}{\partial q_{e}}$ has full rank. The corresponding holonomic constraint is

$$
\eta_{s t, R}\left(q_{e}\right)=\left[\begin{array}{c}
p_{s t} \\
\phi_{s t}^{x} \\
\phi_{s t}^{z}
\end{array}\right]=\text { constant }
$$

and the associated kinematic constraint is

$$
\left[\begin{array}{c}
v_{s t} \\
\omega_{s t}^{x} \\
\omega_{s t}^{z}
\end{array}\right]=J_{s t}\left(q_{e}\right) \dot{q}_{e}=0_{5 \times 1},
$$

where $J_{s t}\left(q_{e}\right)$ consists of the first four rows of $J_{s t}$ in (21) and the sixth one.

\footnotetext{
${ }^{10} \mathrm{D}$ is positive definite and the columns of $B$ remain involutive and linearly independent.
}

Since the rotation along the $y$-axis of $R_{s t}$ is free, the contact wrench has five non-zero components as no moment is exerted about the $y$-axis. The wrench can thus be written

$$
F_{s t}=\left(F_{s t}^{f x}, F_{s t}^{f y}, F_{s t}^{f z}, F_{s t}^{m x}, 0, F_{s t}^{m z}\right)^{\prime} .
$$

Differentiating (33), and using (17), $\ddot{q}_{e}$ and the nonzero components of $F_{s t}$ can be calculated in terms of $q_{e}, \dot{q}_{e}$ and $u$.

The associated constraints to avoid take-off, slipping, and rotation about the $x$-axis of the foot (i.e., rotation in the frontal plane) are

$$
\begin{aligned}
F_{s t}^{f z} & >0 \\
\left|F_{s t}^{f x}\right| & <\frac{\mu}{\sqrt{2}} F_{s t}^{f z}, \\
\left|F_{s t}^{f y}\right| & <\frac{\mu}{\sqrt{2}} F_{s t}^{f z}, \\
-l_{b} F_{s t}^{f z}<F_{s t}^{m x} & <l_{a} F_{s t}^{f z} .
\end{aligned}
$$

As before, these equations can be grouped as in (27) and a unilateral constraint can be added as in (28), which leads to a domain of admissibility as in (29). In addition, if the state of the robot belongs to the domain of admissibility, then a simplified model can be deduced. From (32), the set of generalized position variables can be taken as $q_{R}=$ $\left(\phi_{s t}^{y}, q^{\prime}\right)^{\prime}$ and the dynamic model can be expressed as ${ }^{11}$

$$
D_{R}\left(q_{p}\right) \ddot{q}_{R}+C_{R}\left(q_{R}, \dot{q}_{R}\right) \dot{q}_{R}+G_{R}\left(q_{R}\right)=B_{R}(q) u .
$$

The system is always underactuated because no torque is applied about $\phi_{s t}^{y}$.

\subsection{Point-Foot Contact Model}

Consider again the situation in Sect. 4.2 where the foot is flat on the ground and not slipping. If the size of the stance foot is reduced to zero in all dimensions, that is, $L_{a}=L_{b}=l_{a}=l_{b}=h=0$, then the contact with the ground is reduced to a point. This simplifies walking models because there is then only one way for the swing leg to touch the ground, instead of the contact possibly taking place on any edge of the foot.

When realizing a point foot contact as the limiting case of a foot with finite size, two cases can be considered: (a) the (yaw) moment $F_{s t}^{m z}$ goes to zero as well, so the point contact rotates freely about the z-axis; or (b), no limit on $F_{s t}^{m z}$ is imposed and there is no rotational slipping of the stance foot ${ }^{12}$ (i.e., no yaw rotation). The latter case is assumed here.

It is now shown that the stance ankle must be passive (i.e., unactuated) when a point foot model is arrived at by letting the size and mass of the stance foot go to zero ${ }^{13}$. To see this, consider the torque balance at the fictitious ankle. For a massless foot, one has

$$
\begin{aligned}
& u_{1}=F_{s t}^{m x}-L F_{s t}^{f z}+h F_{s t}^{f y} \\
& u_{2}=F_{s t}^{m y}+l F_{s t}^{f z}-h F_{s t}^{f x},
\end{aligned}
$$

${ }^{11} D_{R}$ is positive definite and the columns of $B_{R}$ remain involutive and linearly independent.

12 This is analogous to the assumption that the tangential forces lying in a friction cone continue to imply, even for a point contact, no slipping along the plane of the ground contact.

${ }^{13}$ Consequently, models in the literature which treat point feet robots with actuation at the fictitious ankle are assuming a foot of zero mass, but non-zero size. 
where $l, L$, and $h$ are the distances along the $x, y$ and $z$ axes between the origin of the reference frame $R_{s t}$ and the ankle. From the ZMP conditions in (26), it follows that both $F_{s t}^{m x}$ and $F_{s t}^{m y}$ must be zero when the size of the supporting foot is zero. From (36), we obtain $u_{1}=u_{2}=0$, and therefore the stance ankle joint must be passive.

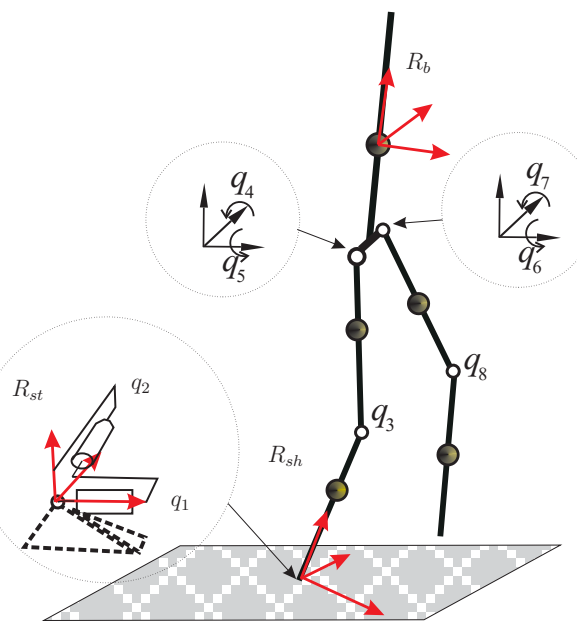

Fig. 4. A reference frame is attached to the shin $R_{s h}$. The motion of the stance shin is limited to the motion produce by a fictitious stance ankle with degrees of freedom $q_{1}=\phi_{s h}^{x}$ and $q_{2}=\phi_{s h}^{y}$.

With the foot reduced to a point, the reference frame used to define the contact constraint is moved to the end of the leg, with its $z$-axis aligned along the shin as depicted in Fig. 4. The reference frame is relabeled as $R_{s h}$; its position and orientation are denoted by $p_{s h}$ and $\phi_{s h}$, respectively. In order to simplify the definition of the holonomic constraint, we chose to define the angles such that the orientation of the frame $R_{s h}$ with respect to the frame $R_{0}$ is ${ }^{0} A_{s h}=\operatorname{Rot}\left(z, \phi_{s h}^{z}\right) \operatorname{Rot}\left(x, \phi_{s h}^{x}\right) \operatorname{Rot}\left(y, \phi_{s h}^{y}\right)$. Consequently, the angle $\phi_{s h}^{z}$ defines the orientation of a fictitious foot, and $\phi_{s h}^{x}$ and $\phi_{s h}^{y}$ are the angles of the fictitious ankle. Recalling that we assume no yaw rotation for the stance leg end, the appropriate holonomic constraint is

$$
\eta_{s h}\left(q_{e}\right)=\left[\begin{array}{l}
p_{s h}\left(q_{e}\right) \\
\phi_{s h}^{z}\left(q_{e}\right)
\end{array}\right]=\text { constant. }
$$

It can be shown that the Jacobian $\frac{\partial \eta_{s h}}{\partial q_{e}}$ has full rank.

It is not straightforward to define the contact wrench in the frame $R_{s h}$ because it does not have an axis perpendicular to the ground, making it impossible to express easily the normal component. Thus a frame $R_{s t}$ linked to the fictitious stance foot ${ }^{14}$ is defined. The contact wrench computed at the origin of $R_{s t}$ has four nonzero components, because the only nontrivial moment is about the $z$-axis. It can thus be written as $F_{s t}=\left(F_{s t}^{f x}, F_{s t}^{f y}, F_{s t}^{f z}, 0,0, F_{s t}^{m z}\right)^{\prime}$.

The kinematic constraint corresponding to the holonomic constraint (37) is

$$
\left[\begin{array}{c}
v_{s t} \\
\omega_{s t}^{z}
\end{array}\right]=J_{s t}\left(q_{e}\right) \dot{q}_{e}=0_{4 \times 1},
$$

\footnotetext{
${ }^{14}$ This frame is defined with respect to the reference frame $R_{0}$ by the position $p_{s h}$ and the angle $\phi_{s h}^{z}$, thus the use of this frame is coherent with the definition of the holonomic constraint (37).
}

where $v_{s t}$ and $\omega_{s t}$ are the linear and angular velocity of the shin expressed in the frame $R_{s t}$, and $J_{s t}\left(q_{e}\right)$ consists of the first three rows of $J_{s t}$ in (21) and the sixth one. Differentiating (38), and using (17), $\ddot{q}_{e}$ and the four nonzero components of $F_{s t}$ can be calculated as a function of $q_{e}, \dot{q}_{e}$, and $u$.

The associated constraints to avoid take-off and slipping of the foot are

$$
\begin{aligned}
& F_{s t}^{f z}>0, \\
& F_{s t}^{f x}<\frac{\mu}{\sqrt{2}} F_{s t}^{f z}, \\
& F_{s t}^{f y}<\frac{\mu}{\sqrt{2}} F_{s t}^{f z} .
\end{aligned}
$$

There is no longer any constraint of the ZMP type and the fictitious ankle is passive. The equations (39) are grouped as in (27) in order to define the domain of admissibility, as in (29).

When the state of the robot and the control belong to the domain of admissibility, a simplified model is once again deduced. From (37), the set of generalized coordinates can be taken as $q_{P}=\left(\phi_{s h}^{x}, \phi_{s h}^{y}, q^{\prime}\right)^{\prime}$ and the dynamic model can be expressed as ${ }^{15}$

$$
D_{P}\left(q_{p}\right) \ddot{q}_{P}+C_{P}\left(q_{P}, \dot{q}_{P}\right) \dot{q}_{P}+G_{P}\left(q_{P}\right)=B_{P}(q) u .
$$

The system is always underactuated because no torque is applied about $\phi_{s h}^{x}$ and $\phi_{s h}^{y}$.

\subsection{Double support phase}

If both legs are in contact with the ground simultaneously, a double support phase occurs. The ground reaction wrench and constraints on it must be considered for each leg, leading to

$$
D_{e}\left(q_{e}\right) \ddot{q}_{e}+H_{e}\left(q_{e}, \dot{q}_{e}\right)+J_{1}\left(q_{e}\right)^{\prime} F_{1 s t}+J_{2}\left(q_{e}\right)^{\prime} F_{2 s t}=B_{e}(q) u,
$$

where $J_{i}$ is the Jacobian matrix corresponding to leg $i, i=1,2$. The appropriate holonomic and kinematic constraints associated with how each leg is contacting the ground must be considered, and they can be different for each leg. As in Sect. 4.2 - 4.4 , the type of contact determines the rank of the constraints, which is the same as the number of nonzero components in $F_{1 s t}$ and $F_{2 s t}$, denoted $c_{1}$ and $c_{2}$, respectively. We can group all these constraints and reaction forces into the form (27) and add a unilateral constraint, if present, as in (28). The domain of admissibility is then given as in (29).

Remark 11. If the number of independent velocity constraints associated to the ground contact denoted $c 12$ $(c 12 \leq c 1+c 2)$ is greater than 6 , then the robot is over actuated. For a desired motion compatible with the constraints in the robot, an infinite number of control input and reaction wrenches can produce the motion. Control input and reaction wrenches are connected by the dynamic model (41), and control inputs have to be defined such that the reaction wrench belongs to the domain of admissibility. In the specific case of double support with two point-foot contacts, the 6 constraints are not independent, the system is underactuated, and the biped can rotate along the line linking the two points of contact.

\footnotetext{
${ }^{15} D_{P}$ is positive definite and the columns of $B_{P}$ remain involutive and linearly independent.
} 


\subsection{Impact model}

An impact occurs when a point or collection of points on the robot strikes the ground with a nonzero velocity. Typically, as part of the walking gait design, the contact occurs at either the heel of the swing foot, the sole of the foot, or in the case of a point foot model, the end of the swing leg. The mechanics of contacting bodies is a complicated subject Kozlov and Treshchev [1992], Brogliato [1999]. Various aspects of it have been addressed for tool use in robotic manipulators; see Gorinevsky et al. [1997], Siciliano and Villani [1999] and Sciavicco and Siciliano [1996]. Two approximate representations of the contact wrench (forces and torques) have been pursued in the legged robotics literature. One approach assumes the contact is elastic and attempts to model the contact forces due to the deformation of the contacting bodies as in Wei et al. [1993], Plestan et al. [2003]. While elastic models may conceptually capture the actual physical phenomenon, in practice, they tend to introduce a suite of parameters that cannot be readily identified; one is therefore obliged to "guess" reasonable values, introducing uncertainty ${ }^{16}$ and inaccuracy. An alternative approach ${ }^{17}$ assumes the contact is rigid, that is, inelastic.

In a rigid impact, the contact wrench acts over an infinitesimal interval of time and is modeled as a vector of impulses. During the impact, the biped's configuration variables do not change, but the generalized velocities undergo a jump. Most of the rigid impact models used in the locomotion literature are inspired by Hürmüzlü and Marghitu [1994]. The derivation of the model is based on introducing a contact impulse $\delta f_{i m p}$ into the dynamic model (13),

$$
D_{e}\left(q_{e}\right) \ddot{q}_{e}+H_{e}\left(q_{e}, \dot{q}_{e}\right) \dot{q}_{e}=B_{e}(q) u+J\left(q_{e}\right)^{\prime} \delta f_{i m p} .
$$

Assuming that the actuator torques do not contain impulses, (42) is "integrated" over the "duration" of the impact to obtain

$$
D_{e}\left(q_{e}\right)\left(\dot{q}_{e}^{+}-\dot{q}_{e}^{-}\right)=J\left(q_{e}\right)^{\prime} F_{i m p}
$$

where $F_{i m p}:=\int_{t^{-}}^{t^{+}} \delta f_{i m p}(\tau) d \tau$ is the intensity of the impulsive contact wrench over the infinitesimal impact event, $\dot{q}_{e}^{-}$is the generalized velocity just before the impact, and $\dot{q}_{e}^{+}$is the generalized velocity just after the impact. Equation (43) expresses conservation of generalized momentum; see Hürmüzlü and Marghitu [1994]. In the above, it is assumed that the generalized position does not change during the impact, so $q_{e}^{+}=q_{e}^{-}=q_{e}$.

In (43), $\dot{q}_{e}^{-}$is determined as the limit from the left of the state of the robot just before impact, and is thus known. The post-impact velocity $\dot{q}_{e}^{+}$and impact intensity $F_{i m p}$ are unknown. There are thus more unknowns than relations. Completing the model requires that one make a priori assumptions about the nature of the impact. In

\footnotetext{
${ }^{16}$ A compliant ground contact model has been used in Plestan et al. [2003] to check the robustness of a feedback controller computed on the basis of a rigid model. In this case, the uncertainty in the parameters is less important.

17 The user of any contact representation must keep in mind that it is a model of reality and is therefore approximate. The various tradeoffs between ease of use and accuracy must be assessed on a case-by-case basis.
}

the simplest case ${ }^{18}$, the one analyzed here, the impact model is completed in essentially the same manner that the contact wrench was determined in Sect. 4.2 through Sect. 4.4. Namely, the impact wrench intensity is determined by adding a kinematic constraint on velocity; the form of the constraint is determined by the assumed nature of the impact (flat foot versus heel strike versus point foot, former stance leg releases from the ground versus the robot enters double support, for example); and the validity of the assumed impact much be verified posteriori by checking that the force and torque components of the resulting impact wrench, and possibly the post-impact velocities, satisfy a set of inequalities.

In order to illustrate the process, assume that the impact of the swing leg with the ground occurs on the sole of the foot (i.e., on a flat foot), the impacting foot neither slips nor rotates, and the former stance leg releases from the ground after the impact. The double support phase is then instantaneous and an impulsive wrench exists on the impacting leg only. The appropriate kinematic constraint is consequently given by (21), computed for the swing leg instead of the stance leg. The kinematic constraint completes the impact model by specifying that

$$
J\left(q_{e}\right) \dot{q}_{e}^{+}=0
$$

In combination with (43), these relations yield the overall impact model

$$
\left[\begin{array}{cc}
D_{e} & -J^{\prime} \\
J & 0
\end{array}\right]\left[\begin{array}{c}
\dot{q}_{e}^{+} \\
F_{i m p}
\end{array}\right]=\left[\begin{array}{c}
D_{e} \dot{q}_{e}^{-} \\
0
\end{array}\right] .
$$

The matrix on the left-hand side of (45) is square, and it has full rank as long as $J$ has full rank.

Equation (45) shows that the post-impact velocity $\dot{q}_{e}^{+}$and the impulsive contact wrench $F_{i m p}$ depend linearly on the pre-impact velocity $\dot{q}_{e}^{-}$. Block matrix inversion can be performed using the Schur complement (see Zhang [2005]) to obtain a direct expression for post-impact velocity, namely

$$
\dot{q}_{e}^{+}=\left(I_{(N+6)}-D_{e}^{-1} J^{\prime}\left(J D_{e}^{-1} J^{\prime}\right)^{-1} J\right) \dot{q}_{e}^{-} .
$$

Starting from a reduced model of the robot before impact, for example, $q^{-}, \dot{q}^{-}$determined from (30) or (40), the corresponding holonomic constraint yields the complete state of the robot $q_{e}^{-}, \dot{q}_{e}^{-}$just before impact. Solving for the post-impact velocity (46) and projecting it down to the reduced model of the ensuing phase gives a reduced impact model written in the form

$$
\dot{q}^{+}=\Delta(q) \dot{q}^{-} \text {. }
$$

Almost every paper on legged locomotion contains a version of this equation.

It should be noted that the expression (47), which suppresses the use of the complete state of the robot, hides the fact that the holonomic constraints before and after impact are not identical (at the very least, the former swing leg is now in contact with the ground). It is a crucial step, which is omitted in many papers, to verify that the post-impact velocity and the impulsive contact wrench are compatible with the holonomic constraint assumed after impact. In particular, since no constraint was imposed on

\footnotetext{
${ }^{18}$ A contact event does not necessarily correspond to a holonomic constraint. The foot could slide after impact, for example. Such cases are more difficult to model.
} 
the former stance leg, it must be the case that the vertical component of the post-impact foot velocity is positive (i.e., the foot is lifting from the ground after the impact). Next, the friction pyramid should be verified with $F_{i m p}$ replacing $F_{s t}$ (i.e., the foot is not slipping post-impact), and finally, the ZMP conditions need to be checked for $F_{i m p}$ (i.e., the foot is rotating appropriately or not about one of its edges, post impact). If any of these conditions are violated, then the assumed impact model was invalid, another set of constraints must be posed, and the entire process repeated; see Hürmüzlü and Marghitu [1994].

The models for an impact occurring on the heel or for the impact of a point foot, while assuming an instantaneous double support phase, are developed in the same manner, using velocity constraints on the swing leg that are analogous to (33) and (38) and the appropriate nonzero components of $F_{\text {imp }}$. A detailed derivation for a planar point foot contact can be found in Westervelt et al. [2007]; the $3 \mathrm{D}$ case is very similar as shown in Chevallereau et al. [2009b].

Remark 12. If the former stance leg remains on the ground after the impact, reaction forces and velocity constraints must be applied to both legs, similar to the double support model in Sect. 4.5. It has been observed that it may be difficult to satisfy the constraints (no take-off, no slipping, ZMP condition) associated to each impulsive contact wrench. In particular, Miossec and Aoustin [2006] have shown for the planar robot RABBIT that the former stance leg remains on the ground only in the case of an impactless ${ }^{19}$ contact.

Remark 13. In general, in point feet models, the impacting leg is assumed to stay on the ground, although it can slide in the presence of insufficient friction. A point foot that was in rigid contact before impact can either lift-off, stay put, or slide (if friction is insufficient). Thus, for the impact of a walking biped with point feet, six impact cases have to be considered. In the case of a robot with finite feet, many more cases have to be considered since the foot can be flat on the ground or can be rotating about one of its edges, or even one of its corners. Additional complexity could come in the form of Zeno solutions, where the foot rebounds an infinite number of times over a finite interval; see Or and Ames [2008, 2009], Lamperski and Ames [2008], Goebel et al. [2009]. Typically, control solutions are sought which avoid such behavior.

\subsection{Hybrid Models}

An overall hybrid model of a walking gait is constructed by first enumerating a list of dynamic models, $1 \leq \mathrm{i} \leq N_{p}$, corresponding to allowed phases in the gait. In general, a directed graph of possible transitions among phases must be constructed. When studying periodic gaits, it is much simpler and more common to specify a cyclic graph, that is, a temporal order of the form $1 \rightarrow 2 \rightarrow \cdots \rightarrow N_{p} \rightarrow 1$. By imposing a temporal ordering, we are assuming a priori that the flow intersects a specific edge of the domain; this must be taken into account when a control law is designed and then verified when the model is analyzed for stable

\footnotetext{
${ }^{19}$ If the pre-impact velocity satisfies the assumed post-impact condition of contact, i.e $J \dot{q}_{e}^{-}=0$, the velocity does not change at impact, that is, $\dot{q}_{e}^{+}=\dot{q}_{e}^{-}$, and $F_{i m p}=0$. The motion is called impactless.
}

orbits. Specific examples of cyclic graphs are worked out in Sect. 5 and 6.

In the following, we suppose that phase i corresponds to one of the single support models (30), (35), or (40), or a double support model as discussed in Sect. 4.5. Let the configuration space be $Q_{\mathrm{i}}$ and the state space be $\mathcal{X}_{\mathrm{i}}=T Q_{\mathrm{i}}$. The state variable control model is then

$$
\frac{d}{d t}\left[\begin{array}{c}
q_{\mathrm{i}} \\
\dot{q}_{\mathrm{i}}
\end{array}\right]=\left[\begin{array}{c}
\dot{q}_{\mathrm{i}} \\
-D_{\mathrm{i}}^{-1}\left(q_{\mathrm{i}}\right) H_{\mathrm{i}}\left(q_{\mathrm{i}}, \dot{q}_{\mathrm{i}}\right)
\end{array}\right]+\left[\begin{array}{c}
0 \\
D_{\mathrm{i}}^{-1}\left(q_{\mathrm{i}}\right) B_{\mathrm{i}}\left(q_{\mathrm{i}}\right)
\end{array}\right] u_{\mathrm{i}},
$$

where $u_{\mathrm{i}} \in \mathcal{U}_{\mathrm{i}} \subset \mathbb{R}^{m_{\mathrm{i}}}$ is the vector of actuator torques. Defining $x_{\mathrm{i}}=\left(q_{\mathrm{i}}^{\prime}, \dot{q}_{\mathrm{i}}^{\prime}\right)^{\prime}$ yields

$$
\dot{x}_{\mathrm{i}}=f_{\mathrm{i}}\left(x_{\mathrm{i}}\right)+g_{\mathrm{i}}\left(x_{\mathrm{i}}\right) u_{\mathrm{i}}
$$

Recall that the various Lagrangian models come with a domain of admissibility (29) arising from the ground contact conditions. Let the element from the constraint vector (28) corresponding to the appropriate edge for transition into phase $\mathrm{i}+1$ be $H_{\mathrm{i}}^{\mathrm{i}+1}\left(x_{\mathrm{i}}, u\right)$. In general, the transition condition depends on the actuator torques as well as the system's state. For simplicity, it is often supposed that $H_{\mathrm{i}}^{\mathrm{i}+1}$ and $\dot{H}_{\mathrm{i}}^{\mathrm{i}+1}$ do not depend on $u$ so that a switching surface is given by

$$
\mathcal{S}_{\mathrm{i}}^{\mathrm{i}+1}=\left\{x_{\mathrm{i}} \in \mathcal{X}_{\mathrm{i}} \mid H_{\mathrm{i}}^{\mathrm{i}+1}\left(x_{\mathrm{i}}\right)=0, \dot{H}_{\mathrm{i}}^{\mathrm{i}+1}\left(x_{\mathrm{i}}\right)<0\right\} .
$$

Note that as in Sect. 3.3, addition modulo the number of phases $N_{p}$ is used, so that $N_{p}+1=1$.

To complete the specification of a hybrid model, the reset map $\Delta_{\mathrm{i}}^{\mathrm{i}+1}: \mathcal{S}_{\mathrm{i}}^{\mathrm{i}+1} \rightarrow \mathcal{X}_{\mathrm{i}+1}$ must be defined. If the transition condition in (50) corresponds to an impact, such as the swing leg height above the ground going to zero, then the reset map is computed as in (47). In other cases, when impacts are not involved, the transition map is typically determined by inserting the state of the robot into the state space of the full model (13), and then projecting down to the state space of the reduced model of the next phase.

Putting all of this together results in a hybrid control system of the form

$$
\Sigma:\left\{\begin{array}{c}
\mathcal{X}=\left\{\mathcal{X}_{\mathrm{i}}\right\}_{\mathrm{i}=1}^{N_{p}}: \mathcal{X}_{\mathrm{i}} \subset \mathbb{R}^{n_{\mathrm{i}}} \\
\mathcal{U}=\left\{\mathcal{U}_{\mathrm{i}}\right\}_{\mathrm{i}=1}^{N_{p}}: \mathcal{U}_{\mathrm{i}} \subset \mathbb{R}^{m_{\mathrm{i}}} \\
\mathcal{F} \mathcal{G}=\left\{\left(f_{\mathrm{i}}, g_{\mathrm{i}}\right)\right\}_{\mathrm{i}=1}^{N_{p}}: \dot{x}_{\mathrm{i}}=f_{\mathrm{i}}\left(x_{\mathrm{i}}\right)+g_{\mathrm{i}}\left(x_{\mathrm{i}}\right) u_{\mathrm{i}} \\
\mathcal{S}=\left\{\mathcal{S}_{\mathrm{i}}^{\mathrm{i}+1}\right\}_{\mathrm{i}=1}^{N_{p}}: \mathcal{S}_{\mathrm{i}}^{\mathrm{i}+1}=\left\{x_{\mathrm{i}} \in \mathcal{X}_{\mathrm{i}} \mid H_{\mathrm{i}}^{\mathrm{i}+1}\left(x_{\mathrm{i}}\right)=0,\right. \\
\left.\dot{H}_{\mathrm{i}}^{\mathrm{i}+1}\left(x_{\mathrm{i}}\right)<0\right\} \\
\Delta=\left\{\Delta_{\mathrm{i}}^{\mathrm{i}+1}\right\}_{\mathrm{i}=1}^{N_{p}}: x_{\mathrm{i}+1}^{+}=\Delta_{\mathrm{i}}^{\mathrm{i}+1}\left(x_{\mathrm{i}}^{-}\right) .
\end{array}\right.
$$

As with uncontrolled hybrid models, the hybrid control model can be written in the form of a tuple that is more consistent with the literature on hybrid systems, namely,

$$
\mathscr{H} \mathscr{C}=\Sigma=(\Gamma, \mathcal{X}, \mathcal{U}, \mathcal{S}, \Delta, \mathcal{F} \mathcal{G}) .
$$

For the formal definition of hybrid systems stated in this form, and defined on more general graphs, see Sinnet and Ames [2009a].

Remark 14. A typical hybrid model would include phases for support on both the left and right legs. When studying walking gaits with left-right symmetry, a common "trick" in the field is to develop a model of the robot for one of the legs in contact with the ground, say the left one, and 
then to "swap" or relabel angles after swing leg impact in order to propagate the dynamics for the ensuing step. The interest of doing this is that it reduces the number of phases by half. This "trick" is particularly common and straightforward for planar models. For 3D models, it is a little more involved as one must "flip" the sign on the hip width and "flip" the sign convention for all angles that are not in the sagittal plane. When using this more economical model of a biped, a state relabeling procedure is included as part of the reset map at leg impact.

\section{CONTROLLING UNDERACTUATED BIPEDAL LOCOMOTION VIA VIRTUAL CONSTRAINTS AND HYBRID ZERO DYNAMICS}

This section overviews an approach to achieving asymptotically stable bipedal locomotion in the presence of underactuation. As discussed in Sect. 4, the contact conditions between the robot and the ground are extremely important for the control of a biped. Moreover, the most difficult conditions to satisfy are the ZMP conditions, as illustrated in (26) and (34), for example. With this as motivation, the work in this section is tailored to feedback control of a biped with the point-foot contact model described in Sect. 4.4, corresponding to the limiting case of a robot with feet, as the size of the feet decreases to zero. If this robot can be controlled with no actuation at the point of contact, then flat-footed walking with actuated feet (of any size) can be accomplished with (arbitrarily small) torques that will respect the constraint of no rotation about an edge of the stance foot, thereby removing an important obstacle to previous studies of walking. Once a gait consisting only of point feet walking of a 3D robot can be controlled, then, based on previous work in planar robots in Chevallereau et al. [2008] and Choi and Grizzle [2005], there is good reason to believe that a gait consisting of a more complete walking cycle, heel strike, flat foot, toe roll, can be realized in a stable manner. Indeed, a freely rotating point of contact is potentially more difficult to control than the heel-strike or toe-roll phases of a human walking gait, which correspond to rotation about a single axis instead of two axes. Finally, work in Chevallereau et al. [2008] shows that even in the case of flat-footed walking with an actuated ankle, if the center of pressure of the ground reaction forces on the stance foot is actively controlled in order to avoid foot rotation, the corresponding control problem is underactuated. For these reasons, walking with unactuated point feet presents an interesting test case for any control design methodology.

In the following, a constraint is said to be virtual ${ }^{20}$ if it is achieved through feedback control instead of through physical connections, such as gears or contact conditions with the environment. Virtual constraints can be used to synchronize the evolution of a robot's links throughout a gait. A connection with the now-classical notion of zero dynamics will become clear during the presentation, with one novelty being the extension of the notion of zero dynamics to a class of hybrid models that occur in bipedal locomotion. Virtual constraints and hybrid zero dynamics originated in the study of underactuated, planar

20 The term "virtual constraint" was coined by Carlos Canudas de Wit. bipedal locomotion in Grizzle et al. [2001], Westervelt et al. [2003]; a synthesis of these methods can be found in Westervelt et al. [2007]. The methods are currently being extended to underactuated 3D robots; see Chevallereau et al. [2009b]. The utility of virtual constraints and hybrid zero dynamics has been experimentally verified for planar bipedal robots Chevallereau et al. [2003], Westervelt et al. [2004], Sreenath et al. [2010]. The 3D results are still in their infancy and much work remains to be done.

\subsection{Virtual Constraints}

Any attempt to describe a walking gait, even something as simple as the difference between human-like walking (knees bent forward) and bird-like walking (knees bent backward), or the torso being upright versus leaning forward, leads to a description of the posture or shape of the robot throughout a step. In other words, a description of walking involves at least a partial specification of the path traced out in the configuration space of the robot. Fig. 5 illustrates this idea for the simple case of a planar biped evolving in the sagittal plane. The same idea applies to a 3D robot, with the addition of coordinates parameterizing the robot's evolution in the frontal plane and its yaw rotation.

To formalize this approach to locomotion control, suppose that $\mathcal{O}$ is a periodic orbit corresponding to a walking gait with $N_{p} \geq 1$ continuous phases ${ }^{21}$ (or domains). Let $q=\left(q_{1}, \cdots, q_{N}\right)$ be a set of generalized coordinates for a particular phase $\mathcal{X}_{\mathrm{i}}$ of the gait, and let $h_{0}(q)$ be functions of the generalized coordinates that are to be controlled, such as the knee and hip angles illustrated in Fig. 5. Let $\theta(q)$ be a function of the generalized coordinates that is strictly monotonic ${ }^{22}$ on $\mathcal{O}_{\mathrm{i}}:=\mathcal{O} \cap \mathcal{X}_{\mathrm{i}}$, and express the controlled variables as a function ${ }^{23} h_{d}(\theta)$ so that, on the periodic orbit,

Off the orbit,

$$
\left.\left(h_{0}(q)-h_{d}(\theta)\right)\right|_{\mathcal{O}_{\mathrm{i}}} \equiv 0 \text {. }
$$

$$
y=h(q):=h_{0}(q)-h_{d}(\theta)
$$

is nonzero, and a natural objective is therefore to design a feedback controller that drives $h(q)$ asymptotically to zero. The function $h(q)$, or more correctly, the relation $h(q)=h_{0}(q)-h_{d}(\theta)=0$, is called a virtual constraint. The number of constraints will be assumed equal to the number of inputs in all that follows.

Two questions naturally arise:

An analysis question: For given a given periodic orbit $\mathcal{O}$ and selection of virtual constraints (in general, different for each domain), when will driving $y$ in (53) asymptotically to zero render the orbit stable (resp., asymptotically stable, or exponentially stable)?

A synthesis question: how to design virtual constraints, and feedback controllers that asymptotically impose them,

\footnotetext{
${ }^{21}$ The development here focuses on control with full-state feedback within the continuous phases. Control at the discrete transitions is addressed in Westervelt et al. [2007]. Observer design is discussed in Grizzle et al. [2007].

$22 \dot{\theta}$ strictly positive or negative on the orbit. Functions which commonly satisfy this include the horizontal position of the robot's hips with respect to an inertial frame, or the absolute angle of the line connecting the stance leg end to the hip.

${ }^{23}$ Always possible when $\theta$ is strictly monotonic.
} 

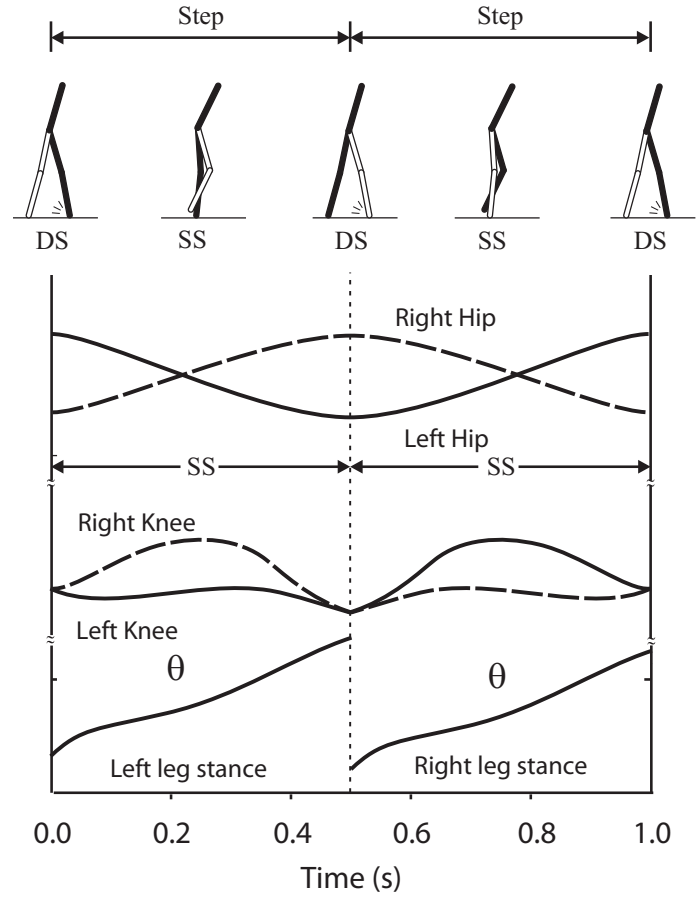

Fig. 5. Illustration of the concept of virtual constraints. Depicted are the relative knee and hip angles versus time for a planar, point-foot walker over a symmetric periodic gait. The gait is comprised of alternating phases of single support (SS), described by a Lagrangian model, and double support (DS), assumed to be instantaneous. The variable $\theta$ (taken here as the angle with respect to the ground of the line connecting the stance leg end to the hip) is strictly monotonically increasing in each continuous phase of the gait. Plotting the joint angles versus $\theta$ provides a graph of the virtual constraints (52) for the relative relative knee and hip angles. The same idea is applicable to 3D walkers.

which together yield an asymptotically stable periodic orbit meeting physically motivated requirements such as: energy efficiency; the robot walks at a desired speed; and the reaction forces at the leg end respect required unilateral constraints?

Addressing the first question leads to the notion of the hybrid zero dynamics, which will be outlined in Sects. 5.2 and 5.3. A finite parametrization of possible paths $h_{d}(\theta, \alpha)$ via Bézier polynomials and parameter optimization have been employed to address the second question; see Sect. 5.4. An illustration is given in Sect. 5.5.

\subsection{Zero Dynamics}

We focus first on the dynamics within a given continuous phase $\mathcal{X}_{\mathrm{i}}$ of a gait model. Let once again $q=\left(q_{1}, \cdots, q_{N}\right)$ be a set of generalized coordinates and suppose the model is given by

$$
D(q) \ddot{q}+C(q, \dot{q}) \dot{q}+G(q)=B u,
$$

where $D(q)$ is the inertia matrix, $C(q, \dot{q})$ contains Coriolis and centrifugal terms, $G(q)$ is the gravity vector, and $B$ is an $N \times N_{a}$ constant matrix with rank $N_{a}<N$. Letting $x=(q, \dot{q})$, and defining $f$ and $g$ in the standard manner, the mechanical model is expressed in state variable form as

$$
\dot{x}=f(x)+g(x) u \text {. }
$$

It is noted in passing that the distribution generated by the columns of $g$ is automatically involutive because the matrix $B$ in (54) is constant.

Let the output be defined as in (53) and assume it has vector relative degree ${ }^{24}$ two. According to Isidori [1995], the zero dynamics manifold is then

$$
\mathcal{Z}_{\mathrm{i}}:=\left\{x \in \mathcal{X}_{\mathrm{i}} \mid h(x)=0, L_{f} h(x)=0\right\} .
$$

A feedback control law rendering $\mathcal{Z}_{\mathrm{i}}$ forward invariant and attractive is

$$
u=u^{*}(x)-\left[L_{g} L_{f} h(x)\right]^{-1}\left(\frac{1}{\epsilon^{2}} K_{p} h(x)+\frac{1}{\epsilon} K_{d} L_{f} h(x)\right)
$$

with

$$
u^{*}(x)=-\left[L_{g} L_{f} h(x)\right]^{-1} L_{f}^{2} h(x),
$$

where $K_{p}>0, K_{d}>0$ and $\epsilon>0$. In applications, $\epsilon>0$ is used to adjust the rate of convergence ${ }^{25}$ to the zero dynamics manifold.

The feedback $u^{*}$ renders $\mathcal{Z}_{\mathrm{i}}$ invariant under the closed-loop vector field $f+g u^{*}$ defined on the continuous phase $\mathcal{X}_{\mathrm{i}}$. The zero dynamics vector field is the restriction

$$
f_{\text {zero }}:=f+\left.g u^{*}\right|_{\mathcal{Z}_{\mathrm{i}}} .
$$

The phase- $\mathcal{X}_{\mathrm{i}}$ zero dynamics is then

$$
\dot{z}=f_{\text {zero }}(z) \text {, }
$$

for $z \in \mathcal{Z}_{\mathrm{i}}$.

Because $\mathcal{Z}_{\mathrm{i}}$ has been designed without consideration of the reset maps in the hybrid model, there is no reason for it to be impact invariant, and hence hybrid invariant. The next section discusses a means developed in Morris and Grizzle [2009] for achieving hybrid invariance without imposing stringent conditions on the feedback designs in neighboring domains or conditions on the reset maps. It is based on realizing the virtual constraints $h$ in (53) as one member of a parameterized family of virtual constraints. The parameters in the constraints are updated upon transition into domain $\mathcal{X}_{\mathrm{i}}$ so as to achieve invariance, while preserving the original orbit used in the design of $h$.

\subsection{Hybrid Invariance}

We continue to focus on a domain $\mathcal{X}_{\mathrm{i}}$ where a feedback law has been constructed as in Sect. 5.2. We suppose for the moment that a feedback law has been defined in each of the other domains; this may be achieved by following the virtual constraints and zero dynamics approach of the

\footnotetext{
${ }^{24}$ The assumption of vector relative degree two is for convenience and works well in applications. A uniform vector relative degree of $k$ is treated in Morris and Grizzle [2009]; a case with a non-uniform vector relative degree is treated in Poulakakis and Grizzle [2009]. Even the assumption of a vector relative degree could be relaxed to dynamic input-output linearizability, for example, but we have had no need to do this in the applications studied to date.

${ }^{25}$ In many concrete examples, the reset map tends to amplify the distance from a periodic orbit and thus the continuous phase dynamics must be sufficiently contractive in order to achieve orbital stability. The parameter $\epsilon$ is used for this purpose. The assumptions on the feedback parameters guarantee that $\lambda^{2}+\frac{1}{\epsilon} K_{d} \lambda+\frac{1}{\epsilon^{2}} K_{p}$ is Hurwitz.
} 
previous section, or by any other approach as long as (i) $\mathcal{O}$ is a periodic orbit of the model and (ii) the closed-loop model is smooth enough for the maps defined below to be $C^{1}$ in a neighborhood of the periodic orbit $\mathcal{O}$. These assumptions allow a system with impulse effects to be associated with domain $\mathcal{X}_{\mathrm{i}}$ by defining

$$
\Sigma:\left\{\begin{array}{rlr}
\dot{x} & =f(x)+g(x) u & x^{-} \notin \mathcal{S} \\
x^{+}=\Delta\left(x^{-}\right) & x^{-} \in \mathcal{S}
\end{array}\right.
$$

where $x \in \mathcal{X}_{\mathrm{i}}, \mathcal{S}=\mathcal{S}_{\mathrm{i}}^{\mathrm{i}+1}$, and $\Delta=\Delta_{\mathrm{i}-1}^{\mathrm{i}} \circ P_{\mathrm{i}-1} \circ \cdots \circ P_{1} \circ$ $P_{N_{p}} \circ \cdots P_{\mathrm{i}+1}$, where $P_{\mathrm{i}}$ are the generalized Poincaré maps defined in $(6)$.

The manifold $\mathcal{Z}_{\mathrm{i}}$ is forward invariant under $f_{\text {zero }}=f+g u^{*}$ and $\mathcal{O}$ is a solution of the zero dynamics. However, $\mathcal{Z}_{i}$ is not necessarily invariant under the transition map $\Delta$, that is, the condition $\Delta\left(\mathcal{Z}_{\mathrm{i}} \cap \mathcal{S}\right) \subset \mathcal{Z}_{\mathrm{i}}$ does not hold in general.

Reference Morris and Grizzle [2009] provides a constructive procedure for determining an open neighborhood $\mathcal{B}$ of the origin in $\mathbb{R}^{2 N_{a}}$, a smooth function $h_{c}: \mathcal{X}_{\mathrm{i}} \times \mathcal{B} \rightarrow \mathbb{R}^{N_{a}}$, and a smooth function $\mathrm{v}: \mathcal{S} \rightarrow \mathcal{B}$ such that

$$
\begin{aligned}
& {\left[\begin{array}{l}
\dot{x} \\
\dot{\beta}
\end{array}\right]=\left[\begin{array}{c}
f(x)+g(x) u \\
0
\end{array}\right]=f_{e}\left(x_{e}\right)+g_{e}(x) u x^{-} \notin \mathcal{S}} \\
& {\left[\begin{array}{l}
x^{+} \\
\beta^{+}
\end{array}\right]=\left[\begin{array}{c}
\Delta\left(x^{-}\right) \\
\mathrm{v}\left(x^{-}\right)
\end{array}\right]=\Delta_{e}\left(x_{e}\right)} \\
& y_{e}=h_{e}\left(x_{e}\right)=h(x)+h_{c}(x, \beta), \\
& x^{-} \in \mathcal{S},
\end{aligned}
$$

with $h$ as defined in (53), satisfies the following properties:

(1) $h_{c}(x, 0) \equiv 0$ all $x \in \mathcal{X}_{\mathrm{i}}$;

(2) $\mathrm{v}\left(x^{*}\right)=0$ where $x^{*}=\overline{\mathcal{O}} \cap \mathcal{S}$ is the fixed point;

(3) $h_{e}$ has the same vector relative degree as $h$ (in this case two) on $\mathcal{X}_{e}=\check{\mathcal{X}}_{\mathrm{i}} \times \mathcal{B}$, where $\check{\mathcal{X}}_{\mathrm{i}}$ is an open neighborhood of $\mathcal{O} \cap \mathcal{X}_{\mathrm{i}}$;

(4) $h_{e}\left(x^{+}, \beta^{+}\right)=0$ and $L_{f_{e}} h_{e}\left(x^{+}, \beta^{+}\right)=0$ for all $x^{-} \in \mathcal{S}$, $x^{+}=\Delta\left(x^{-}\right)$and $\beta^{+}=\mathrm{v}\left(x^{-}\right)$;

(5) there exists an open neighborhood $\check{\mathcal{S}}$ of $\mathcal{S}$ such that, for all $\beta \in \mathcal{B}$ and $x \in \check{\mathcal{S}}, h_{c}(x, \beta)=0$.

System (62) is called a deadbeat hybrid extension. Properties (1) and (2) imply that the set $\mathcal{O}_{e}=\mathcal{O} \times\{0\} \subset \mathcal{X}_{e}$, called the trivial lift of $\mathcal{O}$, is a periodic orbit of (62). Property (3) implies the existence of a (new) zero dynamics manifold and associated restriction dynamics. Property (4) implies that the new zero dynamics manifold is hybrid invariant and contains $\mathcal{O}_{e}$. Property (5) implies that near $\mathcal{S}$, the projection of the new zero dynamics manifold onto $\mathcal{X}_{\mathrm{i}}$ equals the original zero dynamics manifold, $\mathcal{Z}_{\mathrm{i}}$.

Theorem 15. Morris and Grizzle [2009] (Hyrbid Invariance and Orbital Stability) The system (62) in closedloop with the feedback controller defined on $\mathcal{X}_{e}$

$$
\begin{aligned}
u & =u_{e}^{*}-\left[L_{g_{e}} L_{f_{e}} h_{e}\right]^{-1}\left(\frac{1}{\epsilon^{2}} K_{p} h_{e}+\frac{1}{\epsilon} K_{d} L_{f_{e}} h_{e}\right) \\
u_{e}^{*} & =-\left[L_{g_{e}} L_{f_{e}} h_{e}\right]^{-1} L_{f_{e}}^{2} h_{e}
\end{aligned}
$$

with $K_{p}>0, K_{d}>0$, and $\epsilon>0$, satisfies the following properties:

(1) the manifold

$$
\mathcal{Z}_{e}=\left\{x_{e} \in \mathcal{X}_{e} \mid h_{e}\left(x_{e}\right)=0, L_{f_{e}} h_{e}\left(x_{e}\right)=0\right\}
$$

is hybrid invariant;

(2) $\mathcal{O}_{e}$ is a solution of the hybrid zero dynamics;
(3) $(\mathcal{S} \times \mathcal{B}) \cap \mathcal{Z}_{e}=\left(\mathcal{S} \cap \check{\mathcal{Z}}_{\mathrm{i}}\right) \times \mathcal{B}$, where $\check{\mathcal{Z}}_{\mathrm{i}}=\mathcal{Z}_{\mathrm{i}} \cap \check{\mathcal{X}}_{\mathrm{i}}$;

(4) the Poincaré return map for the hybrid zero dynamics $\left.P_{e}^{\epsilon}\right|_{\mathcal{Z}_{e}}:\left(\mathcal{S} \cap \check{\mathcal{Z}}_{\mathrm{i}}\right) \times \mathcal{B} \rightarrow\left(\mathcal{S} \cap \check{\mathcal{Z}}_{\mathrm{i}}\right) \times \mathcal{B}$ has the form

$$
\left.P_{e}^{\epsilon}\right|_{\mathcal{Z}_{e}}(z, \beta)=\left(\rho_{e}^{\epsilon}(z), \mathrm{v}(z)\right),
$$

where $\mathrm{v}$ is the parameter update law of the deadbeat hybrid extension and $\rho_{e}^{\epsilon}: \mathcal{S} \cap \check{\mathcal{Z}}_{\mathrm{i}} \rightarrow \mathcal{S} \cap \check{\mathcal{Z}}_{\mathrm{i}}$; and

(5) there exists $\bar{\epsilon}>0$ such that, for $0<\epsilon<\bar{\epsilon}$, the following are equivalent:

(a) $\mathcal{O}_{e}$ is an exponentially stable periodic orbit

(b) the original fixed point $x^{*}=\overline{\mathcal{O}} \cap \mathcal{S}$ is an exponentially stable fixed point of $\rho_{e}^{\epsilon}$.

\subsection{Gait Design}

The analytical results of Sections 5.1, 5.2, and 5.3 are rendered useful in feedback synthesis by introducing, in each domain $\mathcal{X}_{\mathrm{i}}$ of a biped model, a finite parametrization of the virtual constraints in (53) per

$$
h_{\alpha}(q):=h_{0}(q)-h_{d}(\theta, \alpha)
$$

in concrete applications, we have found it convenient to construct the function $h_{d}$ from Bézier polynomials; see Bézier [1972]. The parameters appearing in (66) introduce free parameters $\alpha$ into the zero dynamics of each domain,

$$
\dot{z}=f_{\text {zero }, \alpha}(z) \text {. }
$$

A fixed order of cycling the phases is postulated: $1 \rightarrow 2 \rightarrow$ $\cdots \rightarrow N_{p} \rightarrow 1$. The search for a periodic walking motion is cast as a constrained nonlinear optimization problem: find parameters which minimize the integral-squared torque per step length ${ }^{26}$,

$$
J=\frac{1}{\text { step length }} \int_{0}^{T}\|u(t)\|_{2}^{2} d t,
$$

where $T$ is the total walking period through the $N_{p}$ phases. The optimization is carried out subject to natural constraints such as the following:

\section{inequality constraints}

- $\theta$ is strictly increasing (i.e, $\dot{\theta}>0$ along the solution of each domain);

- the solution respects the domain of admissibility, (29);

- positive vertical reaction force on the stance foot (a no-take-off constraint) (23);

- a friction constraint (25);

- bounds on allowed actuator torques;

- the swing foot is positioned above the ground in appropriate phases, etc.;

\section{equality constraints}

- conditions at the domain transitions that impose periodicity;

- desired walking speed;

- etc.

The parameters in the optimization include the coefficients in the virtual constraints, initial conditions of the model in the first phase, time spent in each phase, etc. The optimization problem is non-convex in the parameter set, with many local minima, and is very hard to solve. Nevertheless, fmincon in MATLAB and experience gained

\footnotetext{
26 Torque being proportional to current in a DC motor, integralsquared torque is a rough approximation of energy dissipated in the motors.
} 
from studying simple planar robots in Westervelt et al. [2003] have so far allowed approximate solutions to be found for an interesting range of models.

For the purpose of finding a periodic orbit, the zero dynamics is used in each phase. This speeds up the integration of the various mechanical models. For phase-i, the input in (68) is evaluated as

$$
u_{\alpha, \mathrm{i}}^{*}:=-\left.\left[L_{g} L_{f} h_{\alpha}\right]^{-1} L_{f}^{2} h_{\alpha}\right|_{\mathcal{Z}_{\mathrm{i}}},
$$

assuming the virtual constraints have vector relative degree two. Once a periodic orbit is found, a hybrid zero dynamics feedback controller is synthesized via Sect. 5.3, and the stability of the closed-loop hybrid model is evaluated via a Poincaré map as in Theorem 15. The feedback controller is not necessarily synthesized with the same virtual constraints used to find the periodic orbit, though it often is; for details, see Westervelt et al. [2007] and Chevallereau et al. [2009b].

\subsection{Illustration on an Underactuated Biped}

The design of a stabilizing controller for a simple 3D bipedal robot is illustrated here. The influence of the frontal (coronal) plane dynamics on the overall motion of the robot will be emphasized as this is the major new element when passing from $2 \mathrm{D}$ to $3 \mathrm{D}$. With this in mind, the simplest mechanical structure that highlights this aspect of the gait design and stabilization problem will be used.

Biped: The 3D-biped depicted in Fig. 6 is taken from Chevallereau et al. [2009b]. It consists of five links: a torso and two legs with revolute one DOF knees that are independently actuated and terminated with "point-feet". Each hip consists of a revolute joint with two DOF and each DOF is independently actuated. The width of the hips is nonzero. The stance leg is assumed to act as a passive pivot in the sagittal and frontal planes, with no rotation about the z-axis (i.e., no yaw motion). Indeed, the small link in the diagram that appears to form a foot has zero length and no mass. Its purpose is to indicate the two DOF at the leg-ground contact point corresponding to motion in the frontal $\left(q_{1}\right)$ and sagittal $\left(q_{2}\right)$ planes; in addition, it shows that there is no yaw rotation about the stance leg end per Sect. 4.4 and Fig. 4. The angles $q_{1}$ and $q_{2}$ are unactuated. The remaining joints are independently actuated. In single support, the robot is underactuated, having 8 DOF and 6 independent actuators. The physical parameters of the robot are given in Table 1.

Studied Gait: The walking gait consists of phases of single support, alternating on the left and right legs, with transitions determined by the height of the swing leg above the ground becoming zero. The impact of the swing leg with the ground is assumed to be rigid as in Sect. 4.6, and, consequently, the double support phase is instantaneous. Following Sect. 4.4, a dynamic model is easily developed. The hybrid model naturally has two continuous domains, corresponding to single support on the left and right legs, respectively. If a gait is sought that is symmetric with respect to the left and right legs, it is possible (and common) to use a model with a single continuous phase, and to "flip" the sign of the hip width from one step to the next; see Chevallereau et al. [2009b].

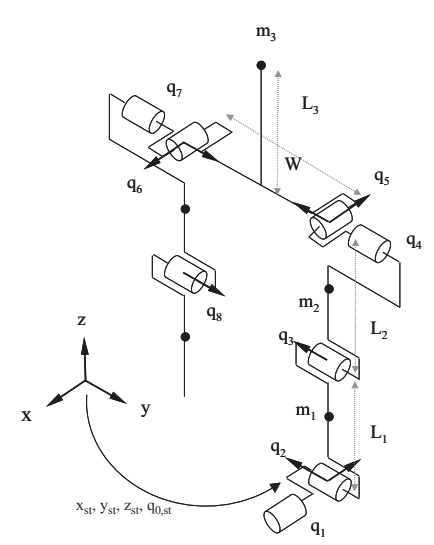

(a)

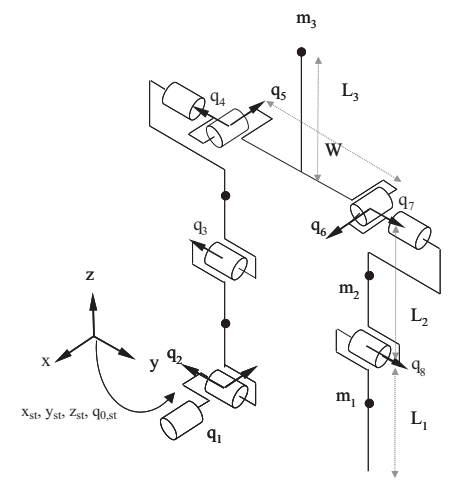

(b)
Fig. 6. A five-link 3D biped with point feet that is 0.55 $\mathrm{m}$ at the hip and has a total mass of $7.25 \mathrm{Kg}$. (a) shows the coordinates for single support on leg-1 and (b) shows the coordinates for single support on leg-2.

\begin{tabular}{|l|l|l|l|l|l|l|l|}
\hline $\mathrm{g}$ & $\mathrm{W}$ & $\mathrm{L} 1$ & $\mathrm{~L} 2$ & $\mathrm{~L} 3$ & $\mathrm{~m} 1$ & $\mathrm{~m} 2$ & $\mathrm{~m} 3$ \\
\hline 9.81 & 0.15 & 0.275 & 0.275 & 0.05 & 0.875 & 0.875 & 5.5 \\
\hline
\end{tabular}

(in MKS).

Periodic Orbit: A symmetric, periodic walking gait was found as in Sect. 5.4. The model has two continuous phases, corresponding to support on leg-1 and then leg2. In each phase, the function $h_{0}(q)$ in (53) was selected as the actuated variables, namely,

$$
h_{0}(q)=\left[\begin{array}{c}
q_{3} \\
\vdots \\
q_{8}
\end{array}\right] .
$$

The variable $\theta$ was taken as

$$
\theta=-q_{2}-q_{3} / 2
$$

which corresponds to the sagittal plane angle of the line connecting the leg end to the hip. Bezier polynomials of degree 3 were chosen for $h_{d}(\theta)$ to complete the definition of the virtual constraints. The parameters were then selected by seeking a (local) minimum of (68).

The computed gait has an average walking speed of 0.75 leg lengths per second; the step length is 0.32 leg lengths; and the step width is close to the hip width. The nominal gait's joint profiles over two consecutive steps are shown in Fig. 7. The unactuated and actuated variables are presented; note that $\theta$ is monotonic over each step. Fig. 8 shows the torque required to produce the periodic motion. Fig. 9 shows the profile of the ground reaction force on the stance foot and the profile of the swing leg end; this figure shows that the unilateral contact constraints are satisfied on the nominal periodic orbit.

Feedback Control: The periodic orbit essentially came with a set of nominal virtual constraints based on the controlled variables (70). Using outputs based on these constraints, a hybrid zero dynamics feedback controller was synthesized following the method of Sect. 5.3. The stability of the closed-loop hybrid model was evaluated via the restricted Poincaré map as in Theorems 8 and 15 . The eigenvalues of the Jacobian of the restricted Poincaré 

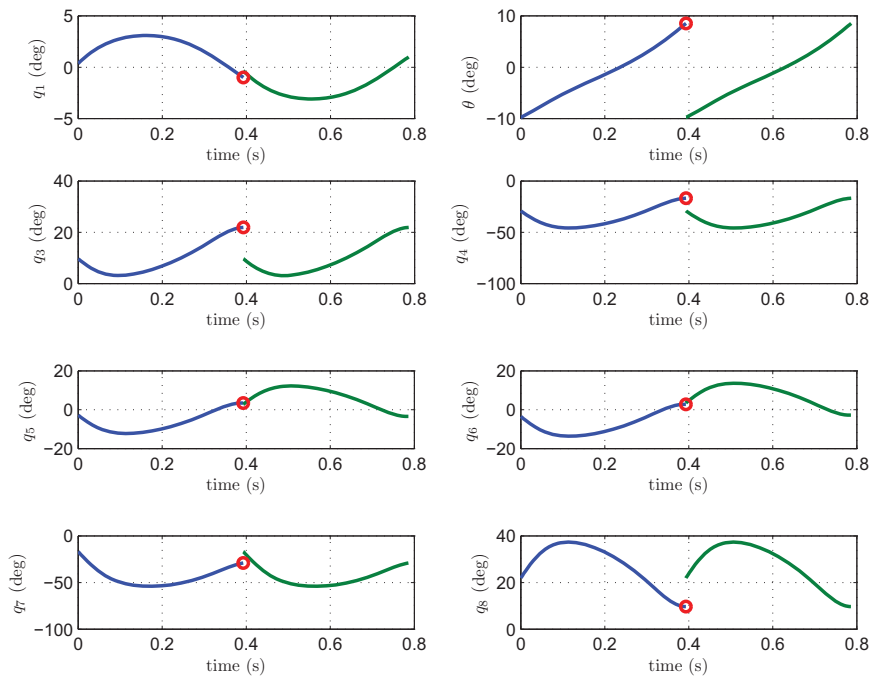

Fig. 7. Joint profiles of a periodic motion that is symmetric over two steps. The small circles represent the points where the discrete transitions occur. Plots of $q_{k}$ versus $\theta$, for $3 \leq k \leq 8$ define the virtual constraints.
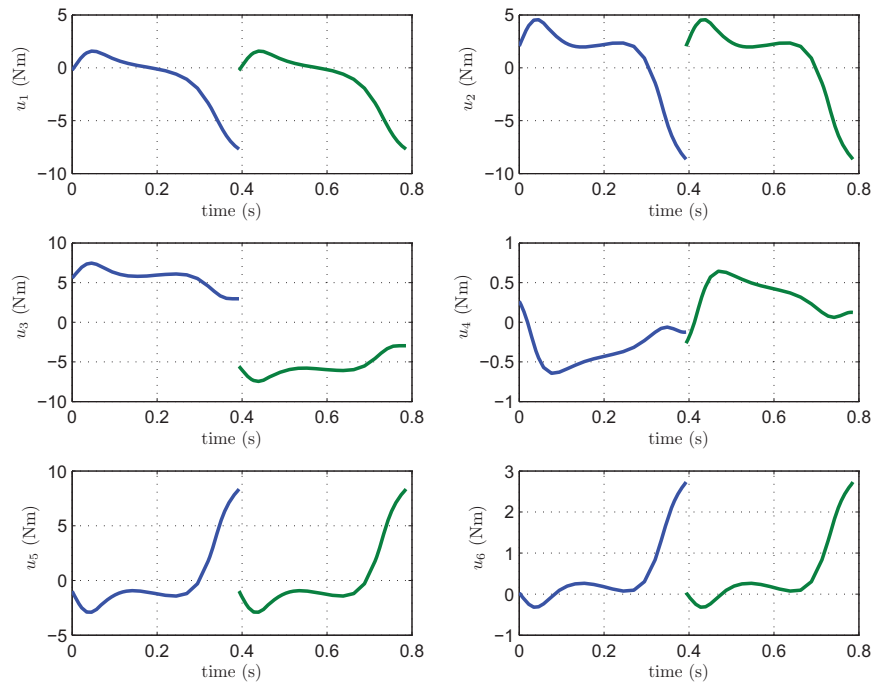

Fig. 8. Torques profiles of the periodic motion over two steps.

map evaluated at the fixed point were

$$
\left|\lambda_{1}\right|=0.89, \quad\left|\lambda_{2}\right|=0.70, \quad\left|\lambda_{3}\right|=2.1
$$

One eigenvalue has magnitude greater than one and hence the gait is unstable under this controller.

An analysis of the eigenvectors did not clearly associate a particular "component" of the state of the HZD with the unstable eigenvalue. Based on results in [Westervelt et al., 2007, pp. 160-163], however, the sagittal plane motion was expected to be stable, so it was suspected that the instability arose in the frontal plane motion. Indeed, the position of the center of mass in the frontal direction is important. If, at leg touchdown, the center of mass is not between the feet, but outside the position of the next supporting foot, the robot will topple sideways. Based on this physical intuition, the control of the variable $q_{6}$ (which regulates step width on the swing leg) was replaced by the control of the distance between the swing leg end and
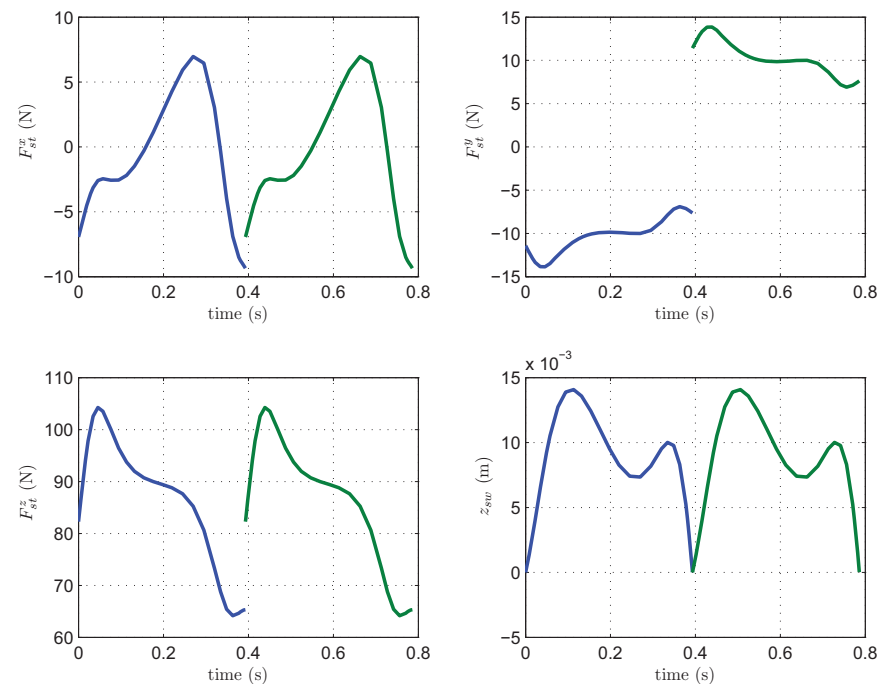

Fig. 9. The reaction forces on the stance foot over two steps and the evolution of the swing leg end. The nominal orbit satisfies the required unilateral constraints at ground contact, as specified in (39).

the center of mass along the frontal direction; denote this distance by $d(q)$.

To incorporate this new objective into the control law, the fourth component of the function $h_{0}(q)$ in the virtual constraints (70) was replaced with ${ }^{27} d(q)$. On the nominal periodic orbit, this distance was evaluated and approximated by a Bezier polynomial in $\theta$, denoted $d^{*}(\theta)$. When the HZD control law of Sect. 5.3 was recomputed using this new output, the walking gait was stable, as shown via the calculation of the eigenvalues of the linearization of the restricted Poincaré map:

$$
\left|\lambda_{1}\right|=0.78, \quad\left|\lambda_{2}\right|=\left|\lambda_{3}\right|=0.25
$$

Remarks on 2D vs. 3D: When extending the method of virtual constraints from planar robots with one degree of underactuation to $3 \mathrm{D}$ robots with two or more degrees of underactuation, new challenges and properties appeared. In the 2D case, hybrid invariance could be achieved without use of a deadbeat hybrid extension. Indeed, Theorem 5.2 in Westervelt et al. [2007] shows that, under very mild conditions, hybrid invariance follows from the nominal virtual constraint vanishing along a periodic orbit. Moreover, the restricted Poincaré map is scalar, can be computed in closed form, and an analytical condition for asymptotic orbital stability can be obtained, based on physical properties of gait along the periodic orbit, and independent of the virtual constraints used to parameterize the orbit. For a robot evolving in 3D, with two or more degrees of underactuation, creating hybrid invariant manifolds is much more challenging. The only known method to achieve invariance under the rest maps is to use a deadbeat hybrid extension. The resulting restricted Poincaré map is of dimension three or higher, and conditions for asymptotic orbital stability depend on the particular choice of the virtual constraints used to parametrize the orbit, as was illustrated here.

${ }^{27} \mathrm{~A}$ linear approximation was in fact used. 


\section{CONTROLLED ROUTHIAN REDUCTION}

Functional Routhian reduction (first introduced in Ames [2006]) is a form of geometric reduction that has been used on numerous 3D bipeds (see, e.g., Ames et al. [2007, 2009], Sinnet and Ames [2009b]) to achieve stable walking. The main idea is that using geometric reduction the bipedal model can be decoupled into its sagittal and coronal dynamics, corresponding to forward and side-to-side motion, respectively. This technique is motivated, as mentioned in Sect. 2, by the large body of work on 2D walking. If the walking can be effectively decoupled, existing control laws can be used on the sagittal portion of the dynamics to achieve stable 2D walking, while the special form of reduction allows for the coronal dynamics to be simultaneously stabilized (the walker will stay upright), resulting in stable $3 \mathrm{D}$ walking.

This section will illustrate this technique and the utility of reduction on a nontrivial 3D biped consisting of multiple discrete phases corresponding to different phases of walking. In particular, we will consider a 3D biped with feet, locking knees, and a hip as in Sinnet and Ames [2010]; this will result in a hybrid model with four phases, two of which are single-support and two of which are double-support. On the single-support phases, functional Routhian reduction will be implemented through the general procedure illustrated in Fig. 6; in fact, this is the procedure that has been utilized on a wide variety of bipedal models implementing this form of control Ames et al. [2006], Ames and Gregg [2007], Sinnet and Ames [2009b], Ames et al. [2007, 2009]. On the double-support phases, local control laws will be used to effect the appropriate phase transitions.

Fig. 10. Proposed scheme for obtaining walking

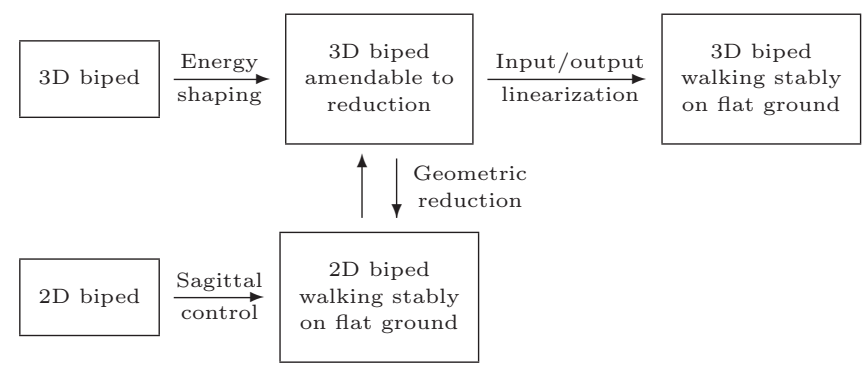

We begin by considering the sagittal restriction of the 3D biped - this model will be a $2 \mathrm{D}$ model operating in the sagittal plane obtained by applying a sagittal-restriction to the 3D model. Sagittal control is applied to this 2D biped to get stable walking in the sagittal plane - arbitrary control laws that yield 2D walking can be used here, but we will consider the specific control law given through controlled symmetries. Once stable 2D sagittal walking has been found, energy shaping is used to transform the Lagrangian of the 3D biped into a form amendable to functional Routhian reduction - the reduced system obtained by applying this form of reduction is just the 2D biped with stable walking. Finally, the decoupling afforded by reduction can only be guaranteed for certain initial conditions. The final control law uses these initial conditions as a "virtual constraint" and uses input/output linearization to stabilize the system to the surface defined

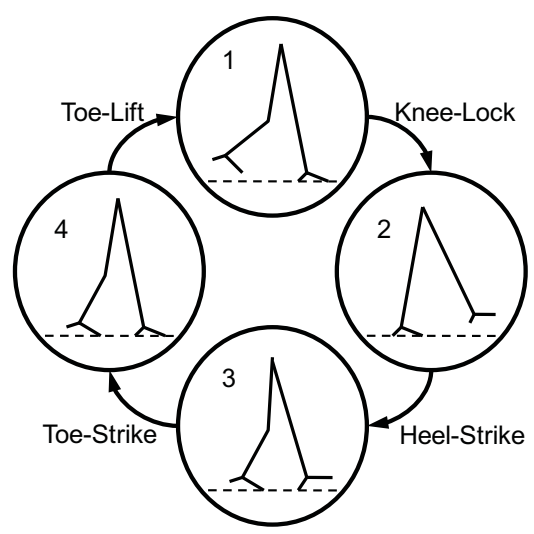

Fig. 11. Directed graph of proposed model

by these conditions. The end result is stable 3D bipedal walking.

\subsection{D Model}

The model of interest in this section is composed of rigid links with point masses as shown in Fig. 12. The model will have configuration space $\mathcal{Q}_{3 \mathrm{D}}$, which will consist of the extended coordinates

$$
q_{e}=\left(p_{b}^{\prime}, \phi_{b}^{\prime}, q^{\prime}\right)^{\prime},
$$

where $p_{b}$ and $\phi_{b}$ are the position and orientation of a point on the body and $q$ represents the joint angles shown in Fig. 13. This will allow us to model each of the four phases of the proposed hybrid model whose discrete structure is shown in Fig. 11. We will go through each phase individually and show how the hybrid system modeling this biped with the chosen discrete structure can be constructed using the methods described in this paper. In particular, we will define the following hybrid control system (as in (51)):

$$
\Sigma_{3 \mathrm{D}}=\left(\Gamma_{3 \mathrm{D}}, \mathcal{X}_{3 \mathrm{D}}, U_{3 \mathrm{D}}, \mathcal{S}_{3 \mathrm{D}}, \Delta_{3 \mathrm{D}}, \mathcal{F} \mathcal{G}_{3 \mathrm{D}}\right),
$$

where here $\Gamma_{3 \mathrm{D}}$ is the oriented graph obtained with vertices $i \in\{1,2,3,4\}$ as pictured in Fig. 11, $\mathcal{X}_{3 \mathrm{D}}=$ $\left\{\mathcal{X}_{3 \mathrm{D}}^{i}\right\}_{i=1}^{4}, U_{3 \mathrm{D}}=\left\{U_{3 \mathrm{D}}^{i}\right\}_{i=1}^{4}, \mathcal{S}_{3 \mathrm{D}}=\left\{\mathcal{S}_{3 \mathrm{D}}^{i \rightarrow i+1}\right\}_{i=1}^{4}, \Delta_{3 \mathrm{D}}=$ $\left\{\Delta_{3 \mathrm{D}}^{i \rightarrow i+1}\right\}_{i=1}^{4}$ and $\mathcal{F} \mathcal{G}_{3 \mathrm{D}}=\left\{\left(f_{3 \mathrm{D}, i}, g_{3 \mathrm{D}, i}\right)\right\}_{i=1}^{4}$. Each of the individual elements of this hybrid model will now be introduced following the procedure in Sect. 4. For each domain, the vector and control field can be calculated from the specified dynamic model following the standard procedure (see Sect. 4).

Phase 1. In this phase, the stance foot interacts with the ground through flat-foot contact and the stance knee is locked. The admissible control is $U_{3 \mathrm{D}}^{1}=\mathbb{R}^{6}$. Associate a frame $R_{s t}^{1}$ to the stance foot as in Sect. 4.2. The contact Jacobian, $J_{s t}^{1}\left(q_{e}\right)$, for flat-foot contact is computed as in (18). To keep the knee locked, we add the holonomic constraint $\eta^{1}\left(q_{e}\right)=q_{3}$ which has Jacobian

$$
J_{\eta}^{1}\left(q_{e}\right)=\frac{\partial}{\partial q_{e}} \eta^{1}\left(q_{e}\right) .
$$

We combine these to get $J^{1}\left(q_{e}\right)=\left(J_{s t}^{1}\left(q_{e}\right)^{\prime}, J_{\eta}^{1}\left(q_{e}\right)^{\prime}\right)^{\prime}$ and use the dynamic model given in (14). Note that the contact constraints can be written in the form (27) which we will soon use to define the domain of admissibility. 


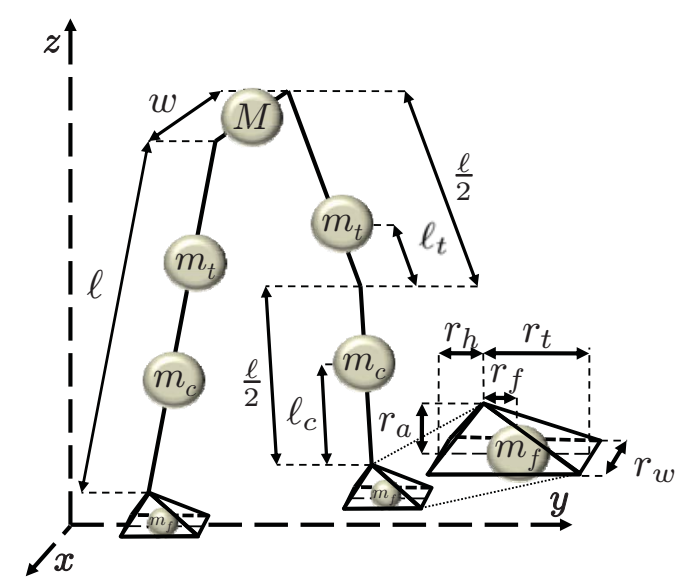

Fig. 12. Configuration of bipedal model

We would like the transition from this phase to be kneelock, which occurs when the swing knee becomes straight and locks. Thus we have the unilateral constraint $h_{1}\left(q_{e}\right)=$ $q_{5}$, which is simply the angle of the swing knee. We combine this unilateral constraint with the constraints in (27) to obtain the constraint vector $H_{1}\left(q_{e}, \dot{q}_{e}\right)$ as in (28). By assuming that the transition is knee-lock, we obtain the switching surface, $\mathcal{S}_{3 \mathrm{D}}^{1 \rightarrow 2}$, given in (50).

We must ensure the validity of the model and the choice of switching surface by computing the domain of admissibility, $D_{1}$, which is given in (29), and verifying that the state of the system belongs to $D_{1}$ as the system passes through this phase. Moreover, $D_{1}$ defines the valid configuration space, i.e., $\mathcal{X}_{3 \mathrm{D}}^{1}=D_{1}$.

Given that the assumptions on friction and foot rotation are satisfied (i.e., the system is operating within the domain of admissibility), we can consider the reduced dynamic model as defined in (30) with reduced coordinates

$$
q_{1}^{r}=\left(q_{2}, q_{4}, q_{5}, q_{6}\right)^{\prime} .
$$

Finally, the reset map $\Delta_{3 \mathrm{D}}^{1 \rightarrow 2}$ corresponding to the impact in the swing knee is found by considering (45) with the kinematic constraint:

$$
J_{1}^{2}\left(q_{e}\right) \dot{q}_{e}=\left(v_{s t}^{\prime}, \omega_{s t}^{\prime}, \dot{q}_{3}, \dot{q}_{5}\right)^{\prime}
$$

We can write the applied impulsive force from (45) as

$$
F_{i m p}=\left(F_{s t}^{f x}, F_{s t}^{f y}, F_{s t}^{f z}, F_{s t}^{m x}, F_{s t}^{m y}, F_{s t}^{m z}, F_{\text {rem }}^{\prime}\right)^{\prime} .
$$

If the force $F_{s t}^{f z}$ is positive, then the stance foot does not leave the ground. If the torque $F_{s t}^{m y}$ is positive, then the stance heel does not leave the ground. Additionally, it must be checked that the stance foot does not slide by verifying that the tangential force is within the friction pyramid as in (25).

Phase 2. Having un-

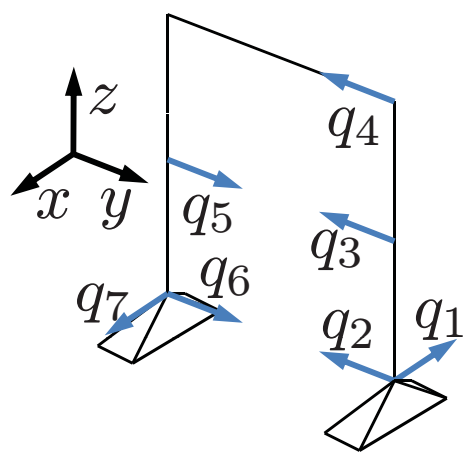

Fig. 13. Configuration of model. Twists are right-handed.

dergone knee-lock as a transition from the previous domain (and having validated the transition assumptions), we still have flat-foot contact of the stance foot and now also have knee-lock of both knees. The admissible control is $\mathcal{U}_{2}=\mathbb{R}^{5}$. As in the previous phase, we associate a frame $R_{s t}^{2}$ to the stance foot which results in the contact Jacobian, $J_{s t}^{2}\left(q_{e}\right)$, for flat-foot contact; this is then computed as in (18). To keep the knees locked we have the holonomic constraint $\eta^{2}\left(q_{e}\right)=\left(q_{3}, q_{5}\right)^{\prime}$ which has Jacobian

$$
J_{\eta}^{2}\left(q_{e}\right)=\frac{\partial}{\partial q_{e}} \eta^{2}\left(q_{e}\right) .
$$

We combine these to get $J^{2}=\left(J_{s t}^{2}\left(q_{e}\right)^{\prime}, J_{\eta}^{2}\left(q_{e}\right)^{\prime}\right)^{\prime}$ and use the dynamic model given in (14). The contact constraints can again be written in the form (27).

The transition from this phase should be heel-strike, which occurs when the swing heel contacts the ground. Upon impact, we will release the lock on the stance knee (and we will soon explain that we will switch legs so this will become the swing knee). Thus, we must associate a frame $R_{s w}^{2}$ to the swing foot, with the origin along the axis of the heel. The appropriate unilateral constraint is $h_{2}\left(q_{e}\right)=p_{s w}^{z}\left(q_{e}\right)$, which is the height of the swing heel above the ground (that is, the height of the origin of $R_{s t}^{2}$.

We combine the mentioned constraints to obtain the constraint vector $H_{2}\left(q_{e}, \dot{q}_{e}\right)$ as in (28). By assuming the transition is heel-strike, we obtain the switching surface, $\mathcal{S}_{3 \mathrm{D}}^{2 \rightarrow 3}$, given in (50).

We compute the domain of admissibility, $D_{2}$, from (29) and use this to define the configuration space: $\mathcal{X}_{3 \mathrm{D}}^{2}=D_{2}$. Given that the system operates within $D_{2}$, we can consider a reduced dynamic model, with reduced coordinates

$$
q_{2}^{r}=\left(q_{1}, q_{2}, q_{4}, q_{6}, q_{7}\right)^{\prime},
$$

given by (30).

We propose that the stance toe does not leave the ground instantaneously as a result of heel-strike but that the stance heel does lift from the ground. In order to verify this, we must first associate a frame $R_{s w}^{2}$ to the swing foot. Since the foot is expected to rotate about the heel, we require that $R_{s w}^{2}$ be aligned with the axis of rotation. Additionally, we require that the previously defined frame $R_{s t}^{2}$ associated to the stance foot be aligned with the axis of rotation (i.e., the stance foot rotates about the toe). We then compute the reset map as in (45) with the constraint Jacobian satisfying

$$
J_{2}^{3}\left(q_{e}\right) \dot{q}_{e}=\left(v_{s t}^{\prime}, \omega_{s t}^{x}, \omega_{s t}^{z}, v_{s w}^{\prime}, \omega_{s w}^{x}, \omega_{s w}^{z}, \dot{q}_{3}, \dot{q}_{5}\right)^{\prime} .
$$

Note that we can write the impulsive force from (45) as

$$
\begin{aligned}
F_{i m p}= & \left(F_{s t}^{f x}, F_{s t}^{f y}, F_{s t}^{f z}, F_{s t}^{m x}, F_{s t}^{m z},\right. \\
& \left.F_{s w}^{f x}, F_{s w}^{f y}, F_{s w}^{f z}, F_{s w}^{m x}, F_{s w}^{m z}, F_{\mathrm{rem}}^{\prime}\right)^{\prime} .
\end{aligned}
$$

We must check that the stance toe does not lift from the ground by verifying that the force $F_{s t}^{f z}$ is positive. In addition, we must check that sliding does not occur upon impact of the swing heel by verifying that the forces $F_{s w}^{f x}$ and $F_{s w}^{f y}$ are within the friction pyramid (25) and we must check that the stance toe does not slide by verifying that the forces $F_{s t}^{f x}$ and $F_{s t}^{f y}$ are within the friction pyramid (25). Finally, we must check that post-impact velocity of $\omega_{s t}^{y}\left(q_{e}\right)$ is negative to verify that the stance heel does indeed lift off the ground. 
As mentioned previously, we introduce a coordinate transformation to "swap" the stance leg and swing leg, allowing us to take advantage of the symmetry of the system. (It should be noted that for certain choice of control, this may result in a bi-periodic orbit as an artifact of modeling.) For the model presented, the new joint angles are given by the following map: $\mathcal{T}_{q}:\left(q_{7}, q_{6}, q_{5},-q_{4}, q_{3}, q_{2}, q_{1}\right) \mapsto$ $\left(q_{1}, q_{2}, q_{3}, q_{4}, q_{5}, q_{6}, q_{7}\right)$. The coordinate transformation for the extended coordinates is simply the identity map. The transformation can therefore be written as a linear map

$$
\mathcal{T}=\left[\begin{array}{cc}
I_{6} & \mathbf{0}_{6 \times 7} \\
\mathbf{0}_{7 \times 6} & \mathcal{T}_{q}
\end{array}\right]
$$

which induces pushforward $\mathcal{T}^{*}$. If all of these conditions hold, then the proposed reset map, $\operatorname{diag}\left(\mathcal{T}, \mathcal{T}^{*}\right) \Delta_{3 \mathrm{D}}^{2 \rightarrow 3}$ computed from (45) - is valid.

Depending on the physical parameters of the model, it can be helpful to assume that the the stance heel lands flat along the edge. The results presented make this assumption which has little effect on the resulting solution due to simulated model parameters. Simple PID control or input/output linearization would allow us to satisfy this assumption for systems where the parameters preclude immediate application of this assumption.

Phase 3. In this phase, we have double support. The admissible control is $\mathcal{U}=\mathbb{R}^{6}$. To achieve double-support and locking of the stance knee, we first associate a frame $R_{s t}^{3}$ to the stance foot and a frame $R_{s w}^{3}$ to the swing foot. Each of these frames must be aligned with the correct axis of rotation as the stance foot is rotating about the heel and the swing foot is rotating about the toe.

Using these frames, we obtain the kinematic constraint:

$$
\begin{aligned}
J_{3}\left(q_{e}\right) \dot{q}_{e}= & \left(v_{s t}\left(q_{e}\right)^{\prime}, \omega_{s t}^{x}\left(q_{e}\right), \omega_{s t}^{z}\left(q_{e}\right),\right. \\
& \left.v_{s w}\left(q_{e}\right)^{\prime}, \omega_{s w}^{x}\left(q_{e}\right), \omega_{s w}^{z}\left(q_{e}\right), \dot{q}_{3}\right)^{\prime} .
\end{aligned}
$$

Enforcing this constraint will ensure locking of the stance knee and the desired double support. The corresponding dynamic model is given in (14).

The transition to the next phase should be toe-strike and, upon impact, the swing toe should remain fixed to the ground. We therefore consider the unilateral constraint, $h_{3}\left(q_{e}\right)=\phi_{s w}^{y}\left(q_{e}\right)$, where $\phi_{s w}^{y}\left(q_{e}\right)$ is the orientation of the swing foot frame $R_{s t}^{3}$ about the heel.

We combine this unilateral constraint with $\eta_{3}\left(q_{e}\right)$ to obtain the constraint vector $H_{3}\left(q_{e}, \dot{q}_{e}\right)$ as in (28). By assuming that the transition is toe-strike, we obtain the switching surface, $\mathcal{S}_{3 \mathrm{D}}^{3 \rightarrow 4}$, given in (50).

We compute the domain of admissibility, $D_{3}$, from (29) and use this to define the configuration space: $\mathcal{X}_{3 \mathrm{D}}^{3}=D_{3}$. Given that the system operates within $D_{3}$, we can consider a reduced dynamic model, with reduced coordinates

given by (30).

$$
q_{3}^{r}=\left(\phi_{s t}^{y}, q_{1}, q_{2}, q_{4}, q_{5}, q_{6}, q_{7}\right)^{\prime}
$$

We propose that the swing toe remains on the ground after impact. This leads us to the kinematic constraint:

$$
\begin{aligned}
J_{3}^{4}\left(q_{e}\right) \dot{q}_{e}= & \left(v_{s t}\left(q_{e}\right)^{\prime}, \omega_{s t}\left(q_{e}\right)^{\prime},\right. \\
& \left.v_{s w}\left(q_{e}\right)^{\prime}, \omega_{s w}^{x}\left(q_{e}\right), \omega_{s w}^{z}\left(q_{e}\right), \dot{q}_{3}\right)^{\prime} .
\end{aligned}
$$

We can write the applied impulsive force from (45) as

$$
\begin{aligned}
F_{i m p}= & \left(F_{s t}^{f x}, F_{s t}^{f y}, F_{s t}^{f z}, F_{s t}^{m x}, F_{s t}^{m y}, F_{s t}^{m z}\right. \\
& \left.F_{s w}^{f x}, F_{s w}^{f y}, F_{s w}^{f z}, F_{s w}^{m x}, F_{s w}^{m z}, F_{\mathrm{rem}}\right)^{\prime}
\end{aligned}
$$

With this constraint we compute the reset map, $\Delta_{3 \mathrm{D}}^{3 \rightarrow 4}$, from (45). In order to validate the proposed transition, we must first check that the force $F_{s w}^{z}$ is positive and that the force applied at $F_{s t}^{z}$ is positive to avoid take-off at these locations. Additionally, we must verify that sliding does not occur by checking that the force in the $x y$-plane at these locations is within the friction pyramid (25).

Phase 4. In this final phase, we still have double-support. The stance foot is now flat on the ground and the swing toe remains on the ground. The admissible control is $\mathcal{U}=\mathbb{R}^{6}$. To model double-support (one foot flat, one foot on edge) and locking of the stance knee, we use use the frames, $R_{s t}^{4}$ and $R_{s w}^{4}$, defined as before, which allows us to obtain the kinematic constraint:

$$
\begin{aligned}
J_{4}\left(q_{e}\right) \dot{q}_{e}= & \left(v_{s t}\left(q_{e}\right)^{\prime}, \omega_{s t}(q e)^{\prime},\right. \\
& \left.v_{s w}\left(q_{e}\right)^{\prime}, \omega_{s w}^{x}\left(q_{e}\right), \omega_{s w}^{z}\left(q_{e}\right), \dot{q}_{3}\right)^{\prime} .
\end{aligned}
$$

The dynamic model is then given in (14).

The transition to the next phase should occur when the swing toe lifts from the ground. Thus, the switching surface, $\mathcal{S}_{3 \mathrm{D}}^{4 \rightarrow 1}$, for this phase is derived from the appropriate element of $\lambda$, the Lagrange multiplier, and given by (51); specifically, it is corresponds to the velocity of the swing toe along the $z$-axis. Because there is no impact, the reset map, $\Delta_{3 \mathrm{D}}^{4 \rightarrow 1}$, for this phase is simply the identity map.

We compute the domain of admissibility, $D_{4}$, from (29) and use this to define the configuration space: $\mathcal{X}_{3 \mathrm{D}}^{4}=D_{4}$. Given that the system operates within $D_{4}$, we can consider a reduced dynamic model, with reduced coordinates

given by (30).

$$
q_{4}^{r}=\left(q_{1}, q_{2}, q_{4}, q_{5}, q_{6}, q_{7}\right)^{\prime},
$$

\subsection{Reduction}

Before constructing the control laws that will be applied to the hybrid control system $\Sigma_{3 \mathrm{D}}$, it is necessary to introduce functional Routhian reduction - the main tool that will be used to achieve three-dimensional walking given walking in two dimensions. This form of reduction utilizes almostcyclic variables, which are analogous to cyclic variables in classical geometric reduction (cf. Marsden and Ratiu [1999]), i.e., these variables are the "symmetries" in the system that will be eliminated through reduction. As with classical reduction, the way in which these variables are eliminated is through a momentum map which describes how momentum is conserved due to the symmetries in the system - the key difference is that in functional Routhian reduction this momentum map is set to be equal to a function of the almost-cyclic variables rather than a constant. This function can be chosen, which will allow us pick a specific function that will stabilize the walker to the upright position while simultaneously decoupling the sagittal and coronal dynamics of the system.

For the model described, we have two almost-cyclic variables $\varphi=\left(q_{1}, q_{6}\right)^{\prime} \in \mathbb{T}^{2}$ and non-conservative external forcing $\Upsilon$ which does not depend on $\varphi$ and does not act on the angles of $\varphi$. We will apply functional Routhian reduction to decouple the sagittal and coronal dynamics of the 
system and then achieve stable walking in 3D by applying reduction control laws and control laws which give stable walking in the sagittally-restricted, $2 \mathrm{D}$ counterpart.

Almost-cyclic Lagrangians. Consider a system with configuration space $\mathcal{Q}=\mathbb{T}^{m} \times S$, where $S$ is called the shape space. Let the coordinates be represented by $q=$ $\left(\varphi^{\prime}, \vartheta^{\prime}\right)^{\prime}$ with $\vartheta \in S$ and almost-cyclic variables $\varphi \in \mathbb{T}^{m}$. A Lagrangian $L_{\lambda}: T \mathbb{T}^{m} \times T S \rightarrow \mathbb{R}$ is almost-cyclic if it takes the form

$$
\begin{aligned}
& L_{\lambda}(\varphi, \vartheta, \dot{\varphi}, \dot{\vartheta})=\frac{1}{2}\left(\begin{array}{ll}
\dot{\varphi}^{\prime} & \dot{\vartheta}^{\prime}
\end{array}\right) D_{\lambda}(\vartheta)\left(\begin{array}{c}
\dot{\varphi} \\
\dot{\vartheta}
\end{array}\right) \\
& -W_{\lambda}(\varphi, \vartheta, \dot{\vartheta})-V_{\lambda}(\varphi, \vartheta) \text {, }
\end{aligned}
$$

with

$$
\begin{gathered}
D_{\lambda}(\vartheta)= \\
\left(\begin{array}{cc}
D_{\varphi}(\vartheta) & D_{\varphi, \vartheta}(\vartheta) \\
D_{\varphi, \vartheta}(\vartheta)^{\prime} & D_{\vartheta}(\vartheta)+D_{\varphi, \vartheta}(\vartheta)^{\prime} D_{\varphi}^{-1}(\vartheta) D_{\varphi, \vartheta}(\vartheta)
\end{array}\right) \\
W_{\lambda}(\varphi, \vartheta, \dot{\vartheta})=\lambda(\varphi)^{\prime} D_{\varphi}^{-1}(\vartheta) D_{\varphi, \vartheta}(\vartheta) \dot{\vartheta} \\
V_{\lambda}(\varphi, \vartheta)=V_{\text {fct }}(\vartheta)-\frac{1}{2} \lambda(\vartheta)^{\prime} D_{\varphi}^{-1}(\vartheta) \lambda(\vartheta)
\end{gathered}
$$

for some function $\lambda: \mathbb{T}^{m} \rightarrow \mathbb{R}^{m}$. Note: $D_{\vartheta}: S \rightarrow$ $\mathbb{R}^{n-m \times n-m}$ and $D_{\varphi}: S \rightarrow \mathbb{R}^{m \times m}$ are positive definite and symmetric.

Momentum maps. Reduction is based on the concept of a momentum map, $J: T \mathcal{Q} \rightarrow \mathbb{R}^{m}$, which specifies the conserved quantities of a system and is given by

$$
\begin{aligned}
J(\varphi, \vartheta, \dot{\varphi}, \dot{\vartheta}) & =\frac{\partial L_{\lambda}(\varphi, \vartheta, \dot{\varphi}, \dot{\vartheta})}{\partial \dot{\varphi}} \\
& =D_{\varphi, \vartheta}(\vartheta) \dot{\vartheta}+D_{\varphi}(\vartheta) \dot{\varphi}
\end{aligned}
$$

Unlike standard Routhian reduction, in which this map is a constant, functional Routhian reduction allows us to set this map equal to a function $\lambda(\varphi)$.

Functional Routhians. For an almost-cyclic Lagrangian $L_{\lambda}$, define the functional Routhian $L_{\mathrm{fct}}: T S \rightarrow$ $\mathbb{R}$ :

$$
L_{\mathrm{fct}}(\vartheta, \dot{\vartheta})=\left[L_{\lambda}(\varphi, \vartheta, \dot{\varphi}, \dot{\vartheta})-\lambda(\varphi)^{\prime} \dot{\varphi}\right]_{J(\varphi, \vartheta, \dot{\varphi}, \dot{\vartheta})=\lambda(\varphi)}
$$

Because $J(\varphi, \vartheta, \dot{\varphi}, \dot{\vartheta})=\lambda(\varphi)$ implies that

$$
\dot{\varphi}=D_{\varphi}^{-1}(\vartheta)\left(\lambda(\varphi)-D_{\varphi, \vartheta}(\vartheta) \dot{\vartheta}\right)
$$

by direct calculation the functional Routhian is given by:

$$
L_{\mathrm{fct}}(\vartheta, \dot{\vartheta})=\frac{1}{2} \dot{\vartheta}^{\prime} D_{\vartheta}(\vartheta) \dot{\vartheta}-V_{\mathrm{fct}}(\vartheta)
$$

Reduction theorem. Before introducing the reduction theorem, note that for $L_{\lambda}$, the forced Euler-Lagrange equations can be written as:

$$
\begin{gathered}
\mathcal{E} \mathcal{L}_{q}\left(L_{\lambda}\right)=D_{\lambda}(\vartheta) \ddot{q}+C_{\lambda}(q, \dot{q}) \dot{q}+ \\
G_{\lambda}(q)+\mathcal{E} \mathcal{L}_{q}\left(W_{\lambda}(q, \dot{q})\right)+\Upsilon(q, \dot{q}),
\end{gathered}
$$

where $C_{\lambda}(q, \dot{q})$ is obtained from $D_{\lambda}, G_{\lambda}(q)=\frac{\partial V_{\lambda}(q)}{\partial q}$, and $\Upsilon(q, \dot{q})$ represents external forcing. Therefore, the forced Euler-Lagrange equations of $L_{\lambda}$ yield the dynamical system:

$$
\begin{aligned}
& f_{L_{\lambda}}(q, \dot{q})= \\
& \quad\left(\begin{array}{l}
\dot{q} \\
D_{\lambda}^{-1}(\vartheta)\left(D_{\lambda}(\vartheta) \ddot{q}-\mathcal{E} \mathcal{L}_{q}\left(L_{\lambda}\right)+\Upsilon(q, \dot{q})\right)
\end{array}\right) .
\end{aligned}
$$

In addition, $f_{L_{\mathrm{fct}}}$, the forced vector field corresponding to $L_{\mathrm{fct}}$, is given by

$$
\begin{aligned}
& f_{L_{\mathrm{fct}}}(\vartheta, \dot{\vartheta})= \\
& \quad\left(\begin{array}{c}
\dot{\vartheta} \\
D_{\vartheta}^{-1}(\vartheta)\left(-C_{\mathrm{fct}}(\vartheta, \dot{\vartheta}) \dot{\vartheta}-G_{\mathrm{fct}}(\vartheta)+\Upsilon(q, \dot{q})\right)
\end{array}\right) .
\end{aligned}
$$

obtained from the controlled Euler-Lagrange equations for $L_{\text {fct }}$, given by: $D_{\vartheta}(\vartheta) \ddot{\vartheta}+C_{\text {fct }}(\vartheta, \dot{\vartheta}) \dot{\vartheta}+G_{\text {fct }}(\vartheta)=\Upsilon(q, \dot{q})$. Note that in the subsequent theorem we will assume that $\Upsilon(q, \dot{q})$ is only dependent on $\vartheta$ and $\dot{\vartheta}$ making equation (79) only a function of $\vartheta$ and $\dot{\vartheta}$, and thus well-defined.

The solutions of these two systems, $f_{L_{\lambda}}$ and $f_{L_{\mathrm{fct}}}$, are related in the following manner (in a way analogous to the classical Routhian reduction result, see [Marsden and Ratiu, 1999, pp. 260]).

Theorem 16. Let $L_{\lambda}$ be an almost-cyclic Lagrangian with almost-cyclic variable $\varphi \in \mathbb{T}^{m}$ and $L_{\mathrm{fct}}$ the corresponding functional Routhian with shape space $S=\mathbb{R}^{n-m}$. Additionally, let $\Upsilon: T S \rightarrow \mathbb{R}^{n}$ represent external forcing satisfying

(i) $\Upsilon(\vartheta, \dot{\vartheta})$ does not depend on $\varphi, \dot{\varphi}$,

(ii) $\Upsilon_{i}(\vartheta, \dot{\vartheta})=0$, for $i \in\{[1, m] \cap \mathbb{Z}\}$.

(I.e., no external forces act on the almost-cyclic variable.)

Then, $(\varphi(t), \vartheta(t), \dot{\varphi}(t), \dot{\vartheta}(t))$ is a solution to the vector field $f_{L_{\lambda}}$ given by $(78)$ on $\left[t_{0}, t_{F}\right]$ with

$$
\dot{\varphi}\left(t_{0}\right)=D_{\varphi}^{-1}\left(\vartheta\left(t_{0}\right)\right)\left(\lambda\left(\varphi\left(t_{0}\right)\right)-D_{\varphi, \vartheta}\left(\vartheta\left(t_{0}\right)\right) \dot{\vartheta}\left(t_{0}\right)\right)
$$

if and only if $(\vartheta(t), \dot{\vartheta}(t))$ is a solution to the forced vector field $f_{L_{\mathrm{fct}}}$ given by $(79)$ and $(\varphi(t), \dot{\varphi}(t))$ satisfies:

$$
\dot{\varphi}(t)=D_{\varphi}^{-1}(\vartheta(t))\left(\lambda(\varphi(t))-D_{\varphi, \vartheta}(\vartheta(t)) \dot{\vartheta}(t)\right) .
$$

Sagittal restriction. To obtain the reduced model, we apply a sagittal restriction, setting all coronal angles to zero, i.e., $q_{1}=0, q_{6}=0$, and projecting down onto the $2 \mathrm{D}$ submanifold. Doing so gives us the hybrid control system:

$$
\Sigma_{2 \mathrm{D}}=\left(\Gamma_{2 \mathrm{D}}, \mathcal{X}_{2 \mathrm{D}}, U_{2 \mathrm{D}}, S_{2 \mathrm{D}}, \Delta_{2 \mathrm{D}}, \mathcal{F} \mathcal{G}_{2 \mathrm{D}}\right),
$$

whereas with the $3 \mathrm{D}$ system, $\Gamma_{2 \mathrm{D}}=\Gamma_{3 \mathrm{D}}$ and $\mathcal{X}_{2 \mathrm{D}}=$ $\left\{\mathcal{X}_{2 \mathrm{D}}^{i}\right\}_{i=1}^{4}, U_{2 \mathrm{D}}=\left\{U_{2 \mathrm{D}}^{i}\right\}_{i=1}^{4}, S_{2 \mathrm{D}}=\left\{\mathcal{S}_{2 \mathrm{D}}^{i \rightarrow i+1}\right\}_{i=1}^{4}, \Delta_{2 \mathrm{D}}=$ $\left\{\Delta_{2 \mathrm{D}}^{i \rightarrow i+1}\right\}_{i=1}^{4}$. Note that for the sagittally-restricted system, $\mathcal{X}_{2 \mathrm{D}}=S$. The vector field and control field on each domain, $\mathcal{F} \mathcal{G}_{2 \mathrm{D}}=\left\{\left(f_{2 \mathrm{D}, i}, g_{2 \mathrm{D}, i}\right)\right\}_{i=1}^{4}$, can be calculated from the dynamic model following the standard procedure (see Sect. 4).

\subsection{Sagittal Control Design}

We will now consider three different controllers that will be combined in a specific manner on each domain to achieve stable walking in the sagittally-restricted $2 \mathrm{D}$ bipedal model. By applying these control laws, we will construct the hybrid system $\bar{\Sigma}_{2 \mathrm{D}}$ and demonstrate through simulation that this model produces stable walking.

Controlled symmetries. The first control law considered is controlled symmetries introduced in Spong and 
Bullo [2005]. This controller works by shaping the potential energy of the associated Lagrangian to that of a passive biped walking down a slope. That is, we effectively "rotate the world" via a group action which operates on the potential energy allowing for walking on flat ground given passive walking down a slope. It was shown in Chen [2007] that a kneed walker can walk passively down a slope given knee-lock and further shown in Ames et al. [2009] that controlled symmetries gives a stable gait for a kneed walker on flat ground. The goal is to combine controlled symmetries with other "local" control laws so as to achieve stable walking in the $2 \mathrm{D}$ sagitally restricted kneed biped with feet.

In order to apply controlled symmetries, consider the group action:

$$
\Psi_{\gamma}(q):=\left(\vartheta_{0}-\gamma, \vartheta_{1}, \ldots\right)^{\prime}
$$

for slope angle $\gamma \in \mathbb{S}$. Using this, we can define the feedback control law:

$$
K_{2 \mathrm{D}}^{\gamma}(\vartheta):=G_{2 \mathrm{D}}(\vartheta)-G_{2 \mathrm{D}}\left(\Psi_{\gamma}(\vartheta)\right)
$$

with $G_{2 \mathrm{D}}(\vartheta)=\frac{\partial V_{2 \mathrm{D}}(\vartheta)}{\partial \vartheta}$.

Controlled symmetries will require full actuation; reduction will require single-support as explained later. For the model under consideration, we will, therefore, only be able to implement controlled symmetries in domains 1 and 2 . Thus, we have the vector fields

$$
f_{2 \mathrm{D}, i}^{\gamma}(\vartheta, \dot{\vartheta})=f_{2 \mathrm{D}, i}(\vartheta, \dot{\vartheta})+g_{2 \mathrm{D}, i}(\vartheta) K_{2 \mathrm{D}, i}^{\gamma}(\vartheta),
$$

for $i \in\{1,2\}$.

Spring-damper controller. Motivated by the elasticity of the human ankle and the need to keep the swing foot from spinning freely when not on the ground, we introduce a spring-damper controller which creates forces on the system equivalent to those of a linear spring-damper system. Consider $j$ relative angles $\Theta: S \rightarrow \mathbb{T}^{j}$ of the system with angular velocities $\dot{\Theta}: T S \rightarrow \mathbb{R}^{j}$ and define the feedback control law:

$$
K_{2 \mathrm{D}}^{\Theta}(\vartheta, \dot{\vartheta}):=B_{\Theta}\left[-k_{\Theta}\left(\Theta(\vartheta)-\Theta_{0}\right)-c_{\Theta} \dot{\Theta}(\dot{\vartheta})\right],
$$

with $k_{\Theta}>0$ a diagonal matrix of spring constants, $c_{\Theta}>0$ a diagonal matrix of viscous damping coefficients, $\Theta_{0}$ the undeflected angles of the springs, and $B_{\Theta}=\left(\frac{\partial \Theta(\vartheta)}{\partial \vartheta}\right)^{\prime}$ a control distribution matrix.

This controller represents a spring-damper system so it will be applied in every domain giving the vector fields

$$
\begin{aligned}
& f_{2 \mathrm{D}, i}^{\gamma, \Theta}(\vartheta, \dot{\vartheta}= \\
& \quad\left\{\begin{array}{l}
f_{2 \mathrm{D}, i}^{\gamma}(\vartheta, \dot{\vartheta})+g_{2 \mathrm{D}, i}^{\Theta}(\vartheta) K_{2 \mathrm{D}, i}^{\Theta}(\vartheta), \text { for } i=1,2, \\
f_{2 \mathrm{D}, i}(\vartheta, \dot{\vartheta})+g_{2 \mathrm{D}, i}^{\Theta}(\vartheta) K_{2 \mathrm{D}, i}^{\Theta}(\vartheta), \text { for } i=3,4,
\end{array}\right.
\end{aligned}
$$

where we simplify notation by superscripting all control gains, even though they are not all used in every domain.

Scuffing prevention controller. The final sagittal control law we consider is designed to prevent scuffing. This controller introduces an effect similar to gravity, but with a repulsive force, defined by

$$
K_{2 \mathrm{D}}^{\mu}(\vartheta)=-\mu_{1} e^{\mu_{2} \cdot p_{s w t}^{z}(\vartheta)}
$$

where $\mu_{1}, \mu_{2} \in \mathbb{R}$ are positive constants, $\mu_{1}$ represents the strength of repulsion, $\mu_{2}$ represents the spatial dissipation
Table 2. Model Parameters and Control Gains

$$
\begin{aligned}
M & =0.5 \mathrm{~kg} \\
m_{t} & =0.5 \mathrm{~kg} \\
m_{c} & =50 \mathrm{mg} \\
m_{f} & =2.5 \mathrm{mg} \\
\gamma & =0.0575 \mathrm{rads} \\
\mu_{1} & =1 \mathrm{Nm}
\end{aligned}
$$$$
\begin{aligned}
\ell & =1 \mathrm{~m} \\
\ell_{t} & =.175 \mathrm{~m} \\
\ell_{c} & =37.5 \mathrm{~cm} \\
r_{h} & =5 \mathrm{~cm} \\
r_{t} & =15 \mathrm{~cm} \\
\mu_{2} & =100 \mathrm{~m}^{-}
\end{aligned}
$$$$
r_{f}=5 \mathrm{~cm}
$$$$
r_{a}=5 \mathrm{~cm}
$$$$
k_{\theta}=2 \mathrm{Nm} / \mathrm{rad}
$$$$
c_{\theta}=0.01 \mathrm{Nms} / \mathrm{rad}
$$$$
\theta_{0}=0^{\circ}
$$

rate, and $p_{s w t}^{z}: S \rightarrow \mathbb{R}$ is the height of the swing toe above the ground. This is applied on domains 1 and 2 (where the swing foot is off the ground) yielding the vector fields

$$
f_{2 \mathrm{D}, i}^{\gamma, \Theta, \mu}(\vartheta, \dot{\vartheta})=f_{2 \mathrm{D}, i}^{\gamma, \Theta}(\vartheta, \dot{\vartheta})+g_{2 \mathrm{D}, i}^{\mu}(\vartheta) K_{2 \mathrm{D}, i}^{\mu}(\vartheta),
$$

for $i \in\{1,2\}$ and

$$
f_{2 \mathrm{D}, i}^{\gamma, \Theta}(\vartheta, \dot{\vartheta})=f_{2 \mathrm{D}, i}^{\gamma, \Theta}(\vartheta, \dot{\vartheta}),
$$

for $i \in\{3,4\}$.

2D simulation. Applying the feedback control laws as shown above to the hybrid control system $\Sigma_{2 \mathrm{D}}$ gives the hybrid system

$$
\bar{\Sigma}_{2 \mathrm{D}}^{\gamma, \Theta, \mu}=\left(\Gamma_{2 \mathrm{D}}, \mathcal{X}_{2 \mathrm{D}}, S_{2 \mathrm{D}}, \Delta_{2 \mathrm{D}}, \mathcal{F}_{2 \mathrm{D}}^{\gamma, \Theta, \mu}\right),
$$

where $\mathcal{F}_{2 \mathrm{D}}^{\gamma, \Theta, \mu}=\left\{f_{2 \mathrm{D}, i}^{\gamma, \Theta}\right\}_{1=1}^{4}$. This hybrid system was simulated (as in Sinnet and Ames [2010]) with model parameters given in Table 2. The resulting gait is shown in Fig. 14. We will examine the stability of the map by considering the codimension-1 Poincaré Section $\mathcal{S}_{1}^{2}$, which is the guard of domain 1 (i.e., knee-lock). Note: on this guard, $q_{1,5}=0$ and $\dot{q}_{1,5}>0$; that is, the knee is becoming locked We find the fixed point:

$$
\begin{aligned}
&\left(q_{1,2}^{r, *}, q_{1,4}^{r, *}, q_{1,6}^{r, *}, \dot{q}_{1,2}^{r, *}, \dot{q}_{1,4}^{r, *}, \dot{q}_{1,5}^{r, *}, \dot{q}_{1,6}^{r, *}\right)= \\
&(0.0503, \quad 0.4583,-0.0032, \\
&1.0845,-0.6635,-9.2966,0.0000)
\end{aligned}
$$

and numerically approximate a linearization of the Jacobian of the Poincaré map to obtain the eigenvalues 0.9526 , $0.2761,0.2761,0.0023,1.2520 \times 10^{-4}, 1.7775 \times 10^{-5}$ and $3.7287 \times 10^{-6}$. These eigenvalues all have magnitude close to but below unity. (The authors have been able to achieve models with maximum eigenvalues of 0.13 by modifying model parameters, however, those results are not presented here.) Therefore, the discrete-time Poincaré map is stable which implies that we have a stable periodic orbit-in other words, we have stable walking.

\subsection{Reduction Control Design}

In order to be able to use the aforementioned 2D control laws, we will need to apply functional Routhian reduction

Fig. 14. Sample gait for sagittally-restricted model
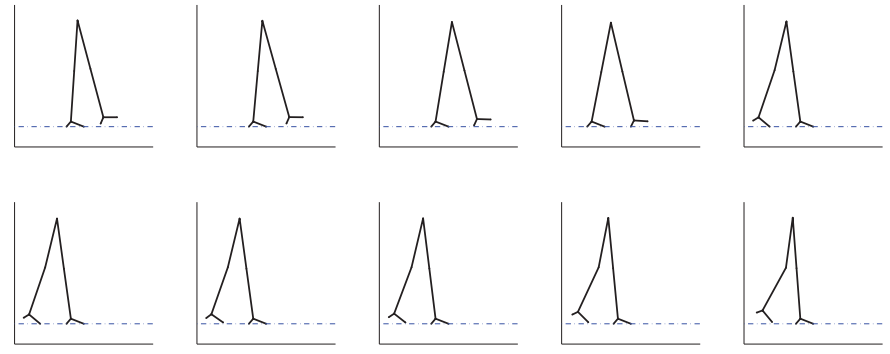


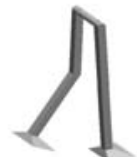

$\Lambda$
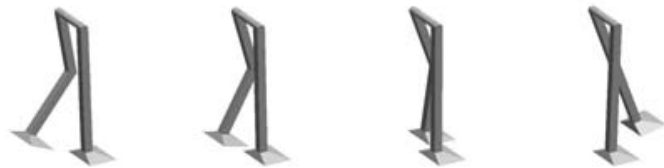

Fig. 15. Sample gait for 3D simulation

to the 3D model via feedback control; doing so will decouple the sagittal and coronal dynamics of the system. Application of controlled Routhian reduction requires not only full actuation, but also single support - a double support model has Lagrange multipliers which both depend on and act (as external forcing) on the almost-cyclic variables. Thus, we can apply reduction only in the first two domains. We will implement the sagittal control through the control law

$$
\begin{aligned}
& K_{3 \mathrm{D}, i}^{\gamma, \Theta}(q, \dot{q}):=B_{2 \mathrm{D}}^{3 \mathrm{D}}\left[B_{\gamma} K_{2 \mathrm{D}, i}^{\gamma}(\pi(\vartheta))+\right. \\
& \left.B_{\Theta} K_{2 \mathrm{D}, i}^{\Theta}(\pi(\vartheta))+B_{\mu} K_{2 \mathrm{D}, i}^{\mu}\left(\pi(\vartheta), \pi^{*}(\dot{\vartheta})\right)\right],
\end{aligned}
$$

where $B_{2 \mathrm{D}}^{3 \mathrm{D}}=\left(\frac{\partial \vartheta}{\partial q}\right)^{\prime}$ and $\pi: \mathcal{X}_{3 \mathrm{D}} \rightarrow S$ is a canonical projection (which induces pushforward $\pi^{*}: T \mathcal{X}_{3 \mathrm{D}} \rightarrow T S$ ) associated to the embedding $\iota: S \rightarrow \mathcal{X}_{3 \mathrm{D}}$, which embeds zeros into the coronal angles. For domain three, this gives the vector field

$$
f_{3 \mathrm{D}, 3}^{\gamma, \Theta, \mu}(q, \dot{q})=f_{3 \mathrm{D}}(q, \dot{q})+g_{3 \mathrm{D}}(q) K_{3 \mathrm{D}, 3}^{\gamma, \Theta, \mu}(q, \dot{q})
$$

Lagrangian shaping controller. Having an almostcyclic Lagrangian enables us to perform reduction. This controller, therefore, shapes the Lagrangian of the system into the almost-cyclic Lagrangian $L_{\alpha}$ of the form (72), where we choose the function $\lambda(\varphi)=-\alpha \varphi$, with $\alpha \in \mathbb{R}$ a positive constant specifying the rate of convergence. Define the control law:

$$
\begin{aligned}
& K_{3 \mathrm{D}}^{\gamma, \Theta, \mu, \alpha}(q, \dot{q}):=B_{3 \mathrm{D}}^{-1}\left(C_{3 \mathrm{D}}(q, \dot{q})+N_{3 \mathrm{D}}(q)+\right. \\
& M_{3 \mathrm{D}}(q) D_{\alpha}^{-1}(\vartheta)\left[D_{\alpha}(\vartheta) \ddot{q}-\mathcal{E} \mathcal{L}_{q}\left(L_{\alpha}(q, \dot{q})\right)+K_{3 \mathrm{D}}^{\gamma, \Theta, \mu}(q, \dot{q})\right]
\end{aligned}
$$

with $D_{\alpha}(\vartheta)$ the shaped inertia matrix as given in (73) and $\mathcal{E} \mathcal{L}_{q}\left(L_{\alpha}(q, \dot{q})\right)$ given in $(77)$. Applying this control law on the first two domains yields the dynamical system:

$$
f_{3 \mathrm{D}, i}^{\gamma, \Theta, \mu, \alpha}(q, \dot{q})=f_{3 \mathrm{D}}(q, \dot{q})+g_{3 \mathrm{D}}(q) K_{3 \mathrm{D}, i}^{\gamma, \Theta, \mu, \alpha}(q, \dot{q}),
$$

for $i \in\{1,2\}$.

Reduction surface stabilization. In order to enjoy the decoupling effects of functional Routhian Reduction, we must stabilize to the surface where reduction is valid. Doing so will result in the system satisfying (81). This motivates the output function

$$
y_{z}(q, \dot{q})=\dot{\varphi}+D_{\varphi}^{-1}(\vartheta)\left(\alpha \varphi+D_{\varphi, \vartheta}(\vartheta) \dot{\vartheta}\right),
$$

which has relative degree one. Driving this output to zero will drive the system to the forward-invariant surface
Fig. 16. Overlay of 2D and 3D Phase Portraits (Angles and Velocities Relative to Vertical)

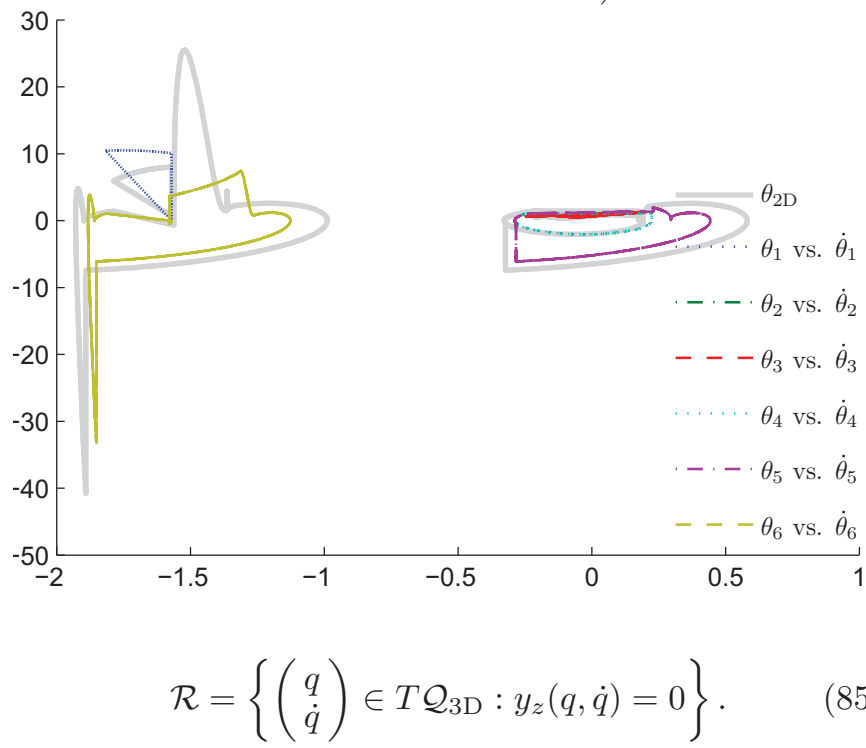

Using MIMO input/output linearization (cf. Sastry [1999]), a control law which results in $y_{z} \rightarrow 0$ is:

$$
K_{3 \mathrm{D}}^{\varepsilon}(q, \dot{q}):=\left[\left(L_{g_{3 \mathrm{D}}} y_{z}\right)^{-1}\left(-L_{f_{3 \mathrm{D}}^{\gamma, \Theta, \mu, \alpha}} y_{z}-\varepsilon y_{z}\right)\right]
$$

with $\varepsilon \in \mathbb{R}$ a positive constant specifying rate of convergence. Applying this control law on domains 1 and 2 gives the closed-loop vector fields

$$
f_{3 \mathrm{D}, i}^{\gamma, \Theta, \mu, \alpha, \varepsilon}(q, \dot{q})=f_{3 \mathrm{D}, i}^{\gamma, \Theta, \mu, \alpha}(q, \dot{q})+g_{3 \mathrm{D}}^{\varepsilon}(q) K_{3 \mathrm{D}}^{\varepsilon}(q, \dot{q}),
$$

for $i \in\{1,2\}$ and

$$
f_{3 \mathrm{D}, i}^{\gamma, \Theta, \mu, \alpha, \varepsilon}(q, \dot{q})=f_{3 \mathrm{D}, i}^{\gamma, \Theta, \mu}(q, \dot{q}),
$$

for $i \in\{3,4\}$.

3D simulation. In this section we present the results of a simulation of the model studied in Sinnet and Ames [2010]. As a result, not all assumptions regarding domain of admissibility are verified. We apply the preceding control laws to the hybrid control system $\Sigma_{3 \mathrm{D}}$ gives the hybrid system

$$
\bar{\Sigma}_{3 \mathrm{D}}^{\gamma, \Theta, \mu, \alpha, \varepsilon}=\left(\Gamma_{3 \mathrm{D}}, \mathcal{X}_{3 \mathrm{D}}, \mathcal{S}_{3 \mathrm{D}}, \Delta_{3 \mathrm{D}}, \mathcal{F}_{3 \mathrm{D}}^{\gamma, \Theta, \mu, \alpha, \varepsilon}\right),
$$

where $\mathcal{F}_{3 \mathrm{D}}^{\gamma, \Theta, \mu, \alpha, \varepsilon}=\left\{f_{3 \mathrm{D}, i}^{\gamma, \Theta, \mu, \varepsilon}\right\}_{i=1}^{4}$. This hybrid system contains the same control laws implemented on the $2 \mathrm{D}$ model and additional reduction control laws. We use the same model parameters and control gains as in the previous simulation and choose the additional control gains $\alpha=10$ and $\varepsilon=25$. The resulting gait is shown in Fig. 15.

In order to examine the stability of the simulated gait, we consider the eigenvalues of the Jacobian of the Poincaré map linearized about a fixed point $\left(q^{*}, \dot{q}^{*}\right) \in T \mathcal{S}_{3 \mathrm{D}}$. For this simulation, we find the fixed point

$$
\begin{gathered}
\left(q_{1,1}^{r, *}, q_{1,2}^{r, *}, q_{1,4}^{r, *}, q_{1,6}^{r, *}, q_{1,7}^{r, *}, \dot{q}_{1,1}^{r, *}, \dot{q}_{1,2}^{r, *}, \dot{q}_{1,4}^{r, *}, \dot{q}_{1,5}^{r, *}, \dot{q}_{1,6}^{r, *}, \dot{q}_{1,7}^{r, *}\right)= \\
(0.000,0.068,-0.367,0.595, \quad 0.000, \\
0.000,0.913, \quad 0.093,6.236,-0.036,0.000) .
\end{gathered}
$$

Due to the numerical complexity of the model, the authors were unable to approximate the eigenvalues of the system. Yet the system appears stable as the trajectory does not diverge from the limit cycle even after hundreds of steps. 
The solutions to the $2 \mathrm{D}$ and $3 \mathrm{D}$ section are shown overlaid in Fig. 16. The main observation here is that the limit cycles are similar. In fact, these limit cycles would be identical if the reset map and guard for the $2 \mathrm{D}$ and $3 \mathrm{D}$ systems were equivalent. However, because of the slight difference in reset maps, the system is thrown off the surface where reduction is valid at each impact. Despite this fact, the system still remains close to the surface and this surface is exponentially stable. Therefore, we have "essentially" decoupled the 3D biped into its sagittal and coronal dynamics through the use of reduction. That is, based on the simulation results, we claim that controlled Routhian reduction can be effectively used to achieving $3 \mathrm{D}$ bipedal walking due to the fact that we are able to "essentially" achieve a sagittal-coronal decoupling. In addition, this technique demonstrates increased efficiency because we have reduced the dimensionality of the system for the purposes of sagittal control design. This is inherently useful due to the fact that a reduction in system dimensionality results in an reduction in complexity.

\section{SUMMARY AND OPEN PROBLEMS}

\subsection{Summary}

This paper has attempted to provide the reader with an introduction to the area of 3D bipedal locomotion. Section 3 emphasized that, in locomotion, steady state behavior corresponds to periodic solutions and not static equilibria. Consequently, Poincaré first return maps are key in characterizing stability of periodic solutions. While some of the technical details associated with Poincaré maps are more delicate for hybrid models, at a fundamental level, the method works essentially the same as for non-hybrid systems described by a single set of ordinary differential equations. The study of periodic solutions of hybrid models was placed before the modeling section for two reasons. First of all, stability is dear to the heart of a control theorist, so its study in hybrid systems would be of interest independent of the underlying physical phenomena. Secondly, it hopefully placed the reader in the frame of mind of seeking to understand in Sect. 4 just how a mixture of continuous and discrete behavior could arise from Lagrangian dynamics.

Section 4 presented models. The models vary depending on both the characteristics of the robot as well as the characteristics of the gait being studied, and this coupling occurs because of the contact forces and moments, called the contact wrench, between the robot and the ground. Because much of the control-oriented literature on locomotion has been ambiguous on the calculation of the contact wrench, the interpretation of its components, and its primal role in determining the validity of a given model, the modeling section paid extra attention to this aspect of bipedal locomotion. The researcher wishing more detail on computing the unconstrained dynamic model of a bipedal robot, that is, the robot without any interactions with its environment other than gravity, can consult numerous texts.

Sections 5 and 6 provided overviews of two approaches to control design for achieving asymptotically stable, periodic walking gaits in 3D bipedal robots. The first approach focused on gaits exhibiting underactuation, while the second focused on gaits composed of a series of phases. The presented work is unabashedly that of the authors. Pointers to work of others were also provided.

\subsection{Challenges}

The paper will conclude with a non-exhaustive list of questions that one or more of the authors find challenging and important.

Aperiodic gaits: The models, analysis procedures and control designs presented in the paper have focused on periodic locomotion. It is important to move beyond this assumption. Some preliminary results can be found in Yang et al. [2007, 2009], Byl and Tedrake [2008a], and Byl and Tedrake [2008b]. Qualitative indicators of stability are discussed in $\mathrm{Su}$ and Dingwell [2007] and references therein, especially in relation to falls and injuries in the elderly. There is room for improved notions of stability of aperiodic walking gaits. Two cases where aperiodic gaits arise naturally are walking on uneven ground and maneuvering a biped around obstacles. These are discussed next.

Walking on uneven ground: This tutorial emphasized walking on flat ground. Much less is known about the problem of bipedal walking on uneven ground. The mechanical design of feet for assuring good ground contact has been studied in Yamaguchi et al. [1995]. Heuristics for maintaining stability on slight inclines were studied in Kim et al. [2007], and for more aggressive ground variations in Hodgins and Raibert [1991], Kajita and Tani [1997], Shih and Chiou [1998], Huang et al. [2000], Shimizu et al. [2007], Erez and Smart [2007]. The role of compliance in locomotion on uneven terrain has been emphasized in Hodgins and Raibert [1991], Saranli et al. [2001], Daley et al. [2006], Daley and Biewener [2006], Hashimoto et al. [2006], Ogino et al. [2007].

Maneuvering: Relatively few papers have addressed the issue of maneuvering for bipedal robots, and even fewer have attempted to provide stability guarantees. Heuristic methods have included turning motions based on the duty ratios of the two legs; allowing the feet to slip when rotating with respect to the ground; other trial-and-error methods Shih [1999], Kazuo et al. [2004], Miura et al. [2008], Yagi and Lumelsky [2000]. The references Gregg and Spong [2008, 2009] have developed an elegant and rigorous setting for stable walking and steering of fully actuated 3D robots using a variant of functional Routhian reduction (the basic ideas behind this form of geometric reduction were discussed in Sec. 6). Steering is achieved by adjusting the yaw set point of the within-stride passivitybased controller. How to turn without violating unilateral ground contact constraints is not explained in this work.

Impact models: Work in Miossec and Aoustin [2002] indicates that the approach currently used to represent an inelastic impact of the swing leg with the ground essentially rules out a nontrivial double support phase. In human walking, the double support phase accounts for approximately $20 \%$ of the gait. A commonly held opinion in the locomotion field is that compliance is essential for achieving such gaits. It would be very useful to find impact 
models, even very approximate ones, that will allow such gaits in robots with "stiff" feet and limbs.

Computation of periodic solutions: The approach in Sect. 5 starts with finding open-loop, periodic solutions to the hybrid model of a bipedal gait. Our experience is that finding periodic solutions of the model is very hard. The problem is even more difficult when seeking solutions that are energy efficient, in addition to being periodic. In order to facilitate the design of a time-invariant, stabilizing controller via the method of virtual constraints and hybrid zero dynamics, we have sought (exact) solutions of the model that were polynomial functions of a variable $\theta(q)$ that is monotonically increasing along a nominal gait. It is unknown if a different class of spline-like functions would yield better solutions. One method to achieve an energy-efficient steady-state behavior for a robot is to find energy-optimal periodic orbits and then render them asymptotically stable.

Computing the Domain of Attraction: Computing the domain of attraction of a stable equilibrium point has been well-studied in the area of dynamical systems. What remains an almost completely unexplored area is computing the domain of attraction for periodic solutions, even for dynamical systems, and in hybrid systems this problem remains completely open. Characterizing the domain of attraction of a periodic walking gait for a bipedal robot model is an important consideration with respect to the eventual implementation of the corresponding feedback controller on the actual biped. There will naturally be errors in the model of the biped, and accounting for these while estimating the domain of attraction would be very useful when passing from simulation to experimentation.

Determining the "Correct" Hybrid Model of a Human-Like Biped: This paper considered two distinct hybrid models. In the literature on bipedal robots, hybrid models have been considered with everywhere from one discrete domain or phase (which is the case for most models considered), to five. This raises the natural question: given an anthropomorphic bipedal robot, what is the hybrid model that best captures the behavior of this system? In particular, what is the model so that when stable walking gaits are found, these gaits are as human-like as possible? Answering this question would be important because, if a single "most human-like" hybrid model could be found, this could be used as the canonical model for studying controller development for obtaining anthropomorphic walking for bipedal robots. Yet, in order to answer this question in a formal manner, it seems necessary to develop a metric that can measure the human-like nature of bipedal walking.

Metrics for Human-Like Walking: There are a wide variety of controllers that can yield walking in a wide array of bipedal robot models; for example, two types of controllers were considered in this paper that yielded walking for two different bipeds. A question is: which controller for which hybrid model yields the most human-like walking? In order to answer this question, a metric on the distance between walking gaits is needed, i.e., a metric for comparing the distance between solutions of hybrid systems even in the case when the hybrid models are different. This could then be used to both compare different controllers- through a comparison of the resulting periodic solutionsand to compare robotic walking with human walkingby computing the distance between periodic solutions for bipedal robots and periodic solutions corresponding to real human walking data (obtained through motion capture, the use of sensors, or a combination of both).

Develop New Cost-Functions: Once a viable metric for comparing walking gaits has been found, it can be used to develop new cost-functions. Cost functions play a fundamental role in developing controllers for bipedal robots since parameters for the controllers are typically found by minimizing a specific cost function, e.g., in the case of hybrid zero dynamics, the parameters in the virtual constraints that are not fixed by the conditions that enforce hybrid invariance are determined by minimizing a cost function. Yet, currently, there are only two cost functions that are typically considered: the integral-squared torque per step length (as was considered in this paper, see (68)) and the specific cost of transport. There is, of course, no guarantee that minimizing these cost functions yields human-like walking. Therefore, through notion of the distance of a specific gait from being human-like, it may be possible to develop better cost functions, i.e., cost functions such that, when minimized, yield periodic solutions that are very "close" to human-like walking gaits. This is potentially a very important problem because it might allow for the development of controllers for bipedal robots that yield more human-like walking gaits and are thus naturally transferable to controllers for prosthetic devices.

Foot shape and prosthetics: Passive robots as studied in Collins et al. [2001], which can stably walk down small slopes under the power of gravity, are inspiring the design of semi-passive robots, which can walk on flat ground with very low energy consumption; see Collins et al. [2005]. Spherically shaped feet have proven especially useful in the design of such robots. With spherical feet, the model of the contact between the ground and the foot is different from the cases studied in this paper, though it involves underactuation similar to the pointfoot contact model. In the case of $2 \mathrm{D}$ walking, the control law proposed in Sect. 5 has been extended to spherical feet in Kinugasa et al. [2009]. The 3D case is open and interesting. Indeed, in human locomotion, as noted in Hansen et al. [2004] and Adamczyk et al. [2006], the stance ankle and foot together approximate the rolling motion of a wheel, imparting energy efficiency to the human gait. This is one of the reasons that foot prosthetics have a spherical shape. In a related line of investigation, Srinivasan et al. [2009a] and Srinivasan et al. [2009b] have tied locomotion models based on virtual constraints to human walking data, for both normal gaits and gaits of transtibial prosthesis users. It is argued that the models can be a useful analytical tool for making more informed design and selection of prosthetic components for arriving at more energy efficiency gaits in prosthesis users. It would be interesting to extend this work to address gait stability in the presence of passive or active prostheses.

Other: Many other interesting questions arise, ranging from reflex actions to enhance stability under large perturbations, to bipedal robot safety when operating around humans, manipulation of objects, navigation, etc. 


\section{REFERENCES}

P. G. Adamczyk, S. H. Collins, and A. D Kuo. The advantages of a rolling foot in human walking. Journal of Experimental Biology, 209:3953-3963, 2006.

A. D. Ames. A Categorical Theory of Hybrid Systems. PhD thesis, University of California, Berkeley, 2006.

A. D. Ames and R. D. Gregg. Stably extending twodimensional bipedal walking to three dimensions. In American Control Conference, pages 177-182, New York, NY, 2007.

A. D. Ames, R. D. Gregg, E. D. B. Wendel, and S. Sastry. Towards the geometric reduction of controlled threedimensional robotic bipedal walkers. In 3rd Workshop on Lagrangian and Hamiltonian Methods for Nonlinear Control (LHMNLC'06), Nagoya, Japan, 2006.

A. D. Ames, R. D. Gregg, and M. W. Spong. A geometric approach to three-dimensional hipped bipedal robotic walking. In 45th Conference on Decision and Control, San Diago, CA, 2007.

A. D. Ames, R. W. Sinnet, and E. D. B. Wendel. Threedimensional kneed bipedal walking: A hybrid geometric approach. In 12th International Conference on Hybrid Systems: Computation and Control, San Francisco, CA, 2009.

S. O. Anderson, M. Wisse, C. G. Atkeson, J. K. Hodgins, G. J. Zeglin, and B. Moyer. Powered bipeds based on passive dynamic principles. In 5th IEEE/RAS International Conference on Humanoid Robots, pages 110-116, 2005.

P. Bézier. Numerical Control: Mathematics and Applications. John Wiley \& Sons, New York, NY, 1972.

B. Brogliato. Nonsmooth Mechanics: Models, Dynamics and Control. Springer, New York, NY, 1999.

K. Byl and R. Tedrake. Metastable walking on stochastically rough terrain. In Proceedings of Robotics: Science and Systems IV, 2008a.

K. Byl and R. Tedrake. Approximate optimal control of the compass gait on rough terrain. In IEEE International Conference on Robotics and Automation, Pasadena, CA, 2008b.

V. F. H. Chen. Passive dynamic walking with knees: A point foot model. Master's thesis, MIT, 2007.

C. Chevallereau, G. Abba, Y. Aoustin, F. Plestan, E. R. Westervelt, C. Canudas-de-Wit, and J. W. Grizzle. RABBIT: A testbed for advanced control theory. IEEE Control Systems Magazine, 23(5):57-79, October 2003.

C. Chevallereau, D. Djoudi, and J. W. Grizzle. Stable bipedal walking with foot rotation through direct regulation of the zero moment point. 25(2):390-401, April 2008.

C. Chevallereau, G. Bessonnet, G. Abba, and Y. Aoustin. Bipedal Robots: Modeling, Design and Walking Synthesis. Wiley/ISTE, New York, NY, 2009a.

C. Chevallereau, J. W. Grizzle, and C.-L. Shih. Asymptotically stable walking of a five-link underactuated $3 \mathrm{D}$ bipedal robot. 25(1):37-50, February 2009b.

J. H. Choi and J. W. Grizzle. Planar bipedal walking with foot rotation. In American Control Conference, pages 4909-4916, Portland, OR, 2005.

S. H. Collins, M. Wisse, and A. Ruina. A 3-d passive dynamic walking robot with two legs and knees. International Journal of Robotics Research, 20:607-615, 2001.
S. H. Collins, A. Ruina, R. Tedrake, and M. Wisse. Efficient bipedal robots based on passive-dynamic walkers. Science, (307):1082-1085, 2005.

M. A. Daley and A. A. Biewener. Running over rough terrain reveals limb control for intrinsic stability. Proceedings of the National Academy of Sciences of the United States of America, 103(42):15681-15686, October 2006.

M. A. Daley, J. R. Usherwood, G. Felix, and A. A. Biewener. Running over rough terrain: guinea fowl maintain dynamic stability despite a large unexpected change in substrate height. The Journal of Experimental Biology, 209:171-187, 2006.

E. Dombre and W. Khalil. Modeling, identification and control of robots. Hermes Sciences, Europe. Paris, France, 2002.

T. Erez and W. D. Smart. Bipedal walking on rough terrain using manifold control. In IEEE/RSJ Conference on Intelligent Systems and Robots, pages 1539-1544, San Diego, CA, October 2007.

A. F. Filippov. Differential equations with discontinuous right hand side. American Mathematical Society Translations, 62:199-231, 1960.

R. Goebel, R. Sanfelice, and A. Teel. Hybrid dynamical systems. IEEE Control Systems Magazine, 29(2):28-93, April 2009.

H. Goldstein, C. Poole, and J. Safko. Classical Mechanics. Addison-Wesley, San Francisco, CA, 3rd edition, 2002.

J. M. Goncalves, A. Megretski, and M. A. Dahleh. Global stability of relay feedback systems. IEEE Transactions on Automatic Control, 46(4):550-562, April 2001.

D. M. Gorinevsky, A. M. Formal'sky, and A. Schneider. Force control of robotic systems. CRC Press, Boca Raton, FL, 1997.

A. Goswami. Postural stability of biped robots and the foot-rotation indicator (FRI) point. International Journal of Robotics Research, 18(6):523-533, June 1999.

R. D. Gregg and M. W. Spong. Reduction-based control with application to three-dimensional bipedal walking robots. In American Control Conference, Seatle, WA, 2008.

R. D. Gregg and M. W. Spong. Reduction-based control of three-dimensional bipedal walking robots. International Journal of Robotics Research, 2009.

J. W. Grizzle, G. Abba, and F. Plestan. Asymptotically stable walking for biped robots: Analysis via systems with impulse effects. IEEE Transactions on Automatic Control, 46:51-64, January 2001.

J. W. Grizzle, J. H. Choi, H. Hammouri, and B. Morris. On observer-based feedback stabilization of periodic orbits in bipedal locomotion. In Proceedings of Methods and Models in Automation and Robotics (MMAR 2007), Szczecin, Poland, 2007.

J. Guckenheimer and P. Holmes. Nonlinear Oscillations, Dynamical Systems, and Bifurcations of Vector Fields, volume 42 of Applied Mathematical Sciences. SpringerVerlag, New York, NY, corrected second printing edition, 1996.

J. Guckenheimer and S. Johnson. Planar hybrid systems. In Hybrid Systems II, Lecture Notes in Computer Science, pages 203-225. Springer-Verlag, 1995.

W. M. Haddad, V. S. Chellaboina, and S. G. Nersesov. Impulsive and Hybrid Dynamical Systems: Stability, Dissipativity, and Control. Princeton University Press, 
Princeton, NJ, 2006.

A. H. Hansen, D. S. Childress, and E. H. Knox. Roll-over shapes of human locomotor systems: Effect of walking speed. Clin. Biomech., 19(4):407-414, 2004.

K. Hashimoto, Y. Sugahara, H. Sunazuka, C. Tanaka, A. Ohta, M. Kawase, H. O. Lim, and A. Takanishi. Biped landing pattern modification method with nonlinear compliance control. In IEEE International Conference on Robotics and Automation, pages 1213-1218, Orlando, FL, May 2006.

K. Hirai, M. Hirose, Y. Haikawa, and T. Takenake. The development of Honda humanoid robot. In Proc. of the IEEE International Conference on Robotics and Automation, Leuven, Belgium, pages 1321-1326, May 1998.

H. Hirukawa, F. Kanehiro, K. Kaneko, S. Kajita, K. Fujiwara, Y. Kawai, F. Tomita, S. Hirai, K. Tanie, T. Isozumi, K. Akachi, T. Kawasaki, S. Ota, K. Yokoyama, H. Handa, Y. Fukase, J. Maeda, Y. Nakamura, S. Tachi, and H. Inoue. Humanoid robotics platforms developed in hrp. Robotics and Autonomous Systems, 48(4):165-75, 2004.

I. A. Hiskens. Stability of hybrid limit cycles: application to the compass gait biped robot. In 40th IEEE Conference on Decision and Control, pages 774-779, Orlando, FL, December 2001.

J. K. Hodgins and M. H. Raibert. Adjusting step length for rough terrain locomotion. IEEE Transactions on Robotics and Automation, 7(3):289-298, June 1991.

P. Holmes, R. J. Full, D. E. Koditschek, and J. Guckenheimer. The dynamics of legged locomotion: Models, analyses, and challenges. SIAM Review, 48(2):207-304, May 2006.

Q. Huang, K. Kaneko, K. Yokoi, S. Kajita, T. Kotoku, N. Koyachi, H. Arai, N. Imamura, K. Komoriya, and K. Tanie. Balance control of a biped robot combining off-line pattern with real-time modification. In IEEE International Conference on Robotics and Automation, pages 3346-3352, San Francisco, CA, April 2000.

Y. Hürmüzlü and D. B. Marghitu. Rigid body collisions of planar kinematic chains with multiple contact points. International Journal of Robotics Research, 13(1):82-92, 1994.

Y. Hürmüzlü, F. Génot, and B. Brogliato. Modeling, stability and control of biped robots - a general framework. Automatica, 40(10):1647-1664, 2004.

A. Isidori. Nonlinear Control Systems. Springer-Verlag, Berlin, third edition, 1995.

S. Kajita and K. Tani. Adaptive gait control of a biped robot based on realtimesensing of the ground profile. Autonomous Robots, 4(3):297-305, 1997.

S. Kajita, M. Morisawa, K. Harada, K. Kaneko, F. Kanehiro, K. Fujiwara, and H. Hirukawa. Biped walking pattern generation by using preview control of zeromoment point. In IEEE International Conference on Robotics and Automation, volume 2, pages 1620-1626, Taipei, Taiwan, 2003.

S. Kajita, T. Nagasaki, K. Kaneko, K. Yokoi, and K. Tanie. A hop towards running humanoid biped. In IEEE International Conference on Robotics and Automation, pages 629-35, New Orleans, LA, 2004.

S. Kazuo, K. Tsuchiya, and K. Tsujita. The intelligent ASIMO system overview and integration. In IEEE
International Conference on Robotics and Automation, pages 3043-3048, New Orleans, LA, 2004.

H. K. Khalil. Nonlinear Systems. Prentice Hall, Upper Saddle River, 2nd edition, 1996.

J. Y. Kim, I. W. Park, and J. H. Oh. Walking control algorithm of biped humanoid robot on uneven and inclined floor. Journal of Intelligent and Robotic Systems, 48:457-484, April 2007.

T. Kinugasa, C. Chevallereau, and Y. Aoustin. Effect of circular arc feet on a control law for a biped. Robotica, 27:621-632, 2009.

V. V. Kozlov and D. V. Treshchev. Billiards: A Genetic Introduction to the Dynamics of Systems with Impacts, volume 89 of Translations of Mathematical Monographs. American Mathematical Society, Providence, RI, 1992.

A. D. Kuo. Stabilization of lateral motion in passive dynamic walking. International Journal of Robotics Research, 18(9):917-930, 1999.

A. D. Kuo. Energetics of actively powered locomotion using the simplest walking model. Journal of Biomechanical Engineering, 124:113-120, 2002.

A. D. Kuo. Choosing your steps carefully. IEEE Robotics and Automation Magazine, 14(2):18-29, June 2007.

A. Lamperski and A. D. Ames. On the existence of Zeno behavior in hybrid systems with non-isolated Zeno equilibria. In IEEE Conf. on Decision and Control, pages 2776-2781, Cancún, Mexico, 2008.

J. Lygeros, K. H. Johansson, S. Simic, J. Zhang, and S. Sastry. Dynamical properties of hybrid automata. IEEE Transactions on Automatic Control, 48:2- 17, 2003.

J. E. Marsden and T. S. Ratiu. Introduction to Mechanics and Symmetry, volume 17 of Texts in Applied Mathematics. Springer, 1999.

T. McGeer. Stability and control of two-dimensional biped walking. Technical Report 1, Center for Systems Science, Simon Fraser University, Burnaby, B.C., Canada, 1988.

T. McGeer. Passive dynamic walking. International Journal of Robotics Research, 9(2):62-82, 1990.

S. Miossec and Y. Aoustin. Walking gait composed of single and double supports for a planar biped without feet. In Conference on Climbing and Walking Robots, Paris, France, 2002.

S. Miossec and Y. Aoustin. Fast motions in Biomechanics and Robotics, chapter Dynamical synthesis of a walking cyclic gait for a biped with point feet, pages 233-252. Lecture Notes in Control and Information Sciences. Springer, Heidelberg, Germany, 2006.

K. Miura, S. Nakaoka, M. Morisawa, K. Harada, and S. Kajita. A friction based twirl for biped robots. In IEEE-RAS International Conference on Humanoid Robots, pages 279-284, Daejeon, Korea, 2008.

B. Morris and J. W. Grizzle. A restricted Poincaré map for determining exponentially stable periodic orbits in systems with impulse effects: Application to bipedal robots. In IEEE Conf. on Decision and Control, Seville, Spain, December 2005. IEEE Press.

B. Morris and J. W. Grizzle. Hybrid invariant manifolds in systems with impulse effects with application to periodic locomotion in bipedal robots. IEEE Transactions on Automatic Control, 54(8):1751-1764, August 2009. 
R. M. Murray, Z. Li, and S. S. Sastry. A Mathematical Introduction to Robotic Manipulation. CRC Press, Boca Raton, FL, 1993.

S. G. Nersesov, V. Chellaboina, and W. M. Haddad. A generalization of Poincaré's theorem to hybrid and impulsive dynamical systems. International Journal of Hybrid Systems, 2, 2002.

M. Ogino, H. Toyama, and M. Asada. Stabilizing biped walking on rough terrain based on the compliance control. IEEE/RSJ Conference on Intelligent Systems and Robots, pages 4047-4052, 29 2007-Nov. 2 2007. doi: 10.1109/IROS.2007.4399627.

Y. Or and A. D. Ames. Stability of Zeno equlibria in Lagrangian hybrid systems. In $4^{\text {' }}$ th IEEE Conference on Decision and Control, Cancún, Mexico, 2008.

Y. Or and A. D. Ames. Existence of periodic orbits in completed lagrangian hybrid systems with non-plastic impacts. In 12th International Conference on Hybrid Systems: Computation and Control, volume 5469 of Lecture Notes in Computer Science, pages 291-305. Springer-Verlag, 2009.

T. S. Parker and L. O. Chua. Practical Numerical Algorithms for Chaotic Systems. Springer-Verlag, New York, NY, 1989

F. Pfeiffer, K. Loffler, and M. Gienger. The concept of jogging Johnnie. In IEEE International Conference on Robotics and Automation, Washington, DC, May 2002.

F. Plestan, J. W. Grizzle, E. R. Westervelt, and G. Abba. Stable walking of a 7-DOF biped robot. 19(4):653-668, August 2003.

I. Poulakakis and J. W. Grizzle. The spring loaded inverted pendulum as the hybrid zero dynamics of an asymmetric hopper. IEEE Transactions on Automatic Control, 54 (8):1779-1793, August 2009.

A. V. Roup, D. S. Bernstein, S. G. Nersesov, W. M. Haddad, and V. Chellaboina. Limit cycle analysis of the verge and foliot clock escapement using impulsive differential equations and Poincare maps. International Journal of Control, 76(17):1685-1698, 2003.

Y. Sakagami, R. Watanabe, C. Aoyama, S. Matsunaga, N. Higaki, and K. Fujimura. The intelligent ASIMO: system overview and integration. In IEEE/RSJ Conference on Intelligent Systems and Robots, pages 2478-83, Lausanne, Switzerland, 2002.

U. Saranli, M. Buehler, and D. E. Koditschek. RHex: A simple and highly mobile hexapod robot. International Journal of Robotics Research, 20(7):616-631, July 2001.

S. S. Sastry. Nonlinear Systems: Analysis, Stability and Control. Springer, New York, NY, 1999.

L. Sciavicco and B. Siciliano. Modeling and Control of Robot Manipulators. McGraw-Hill, New York, NY, 1996.

C.-L. Shih. Ascending and descending stairs for a biped robot. IEEE Transactions on Systems, Man, and Cybernetics, 29(3):255-268, May 1999.

C.-L. Shih and C.-J. Chiou. The motion control of a statically stable biped robot on an uneven floor. IEEE Transactions on Systems, Man, and Cybernetics, Part B, 28(2):244-249, April 1998.

H. Shimizu, Y. Wakazuki, Y. Pan, and K. Furuta. Biped walking robot using a stick on uneven ground. In SICE Annual Conference 2007, Kagawa University, Japan, pages 83-88, September 2007.
B. Siciliano and L. Villani. Robot Force Control. Kluwer Academic Publishers, Hingham, MA, 1999.

R. W. Sinnet and A. D. Ames. 2D bipedal walking with knees and feet: A hybrid control approach. In 48th IEEE Conference on Decision and Control, Shanghai, P.R. China, 2009a.

R. W. Sinnet and A. D. Ames. 3D bipedal walking with knees and feet: A hybrid geometric approach. In 48th IEEE Conference on Decision and Control, Shanghai, P.R. China, 2009b.

R. W. Sinnet and A. D. Ames. An application of geometric reduction to three-dimensional bipedal walkers with knees and feet. Submitted for publication, 2010.

M. W. Spong, S. Hutchinson, and M. Vidyasagar. Robot Modeling and Control. John Wiley \& Sons, 2005.

M.W. Spong and F. Bullo. Controlled symmetries and passive walking,. IEEE Transactions on Automatic Control, 50(7):1025-1031, July 2005.

K. Sreenath, H.W. Park, I. Poulakakis, and J. W. Grizzle. A compliant hybrid zero dynamics controller for stable, efficient and fast bipedal walking on mabel. Pre-print, 2010.

S. Srinivasan, E.R. Westervelt, and A.H. Hansen. A lowdimensional sagittal-plane forward-dynamic model for asymetric gait and its application to study the gait of transtibial prosthesis users. Journal of Biomechanical Engineering, 131, 2009a.

S. Srinivasan, ER Westervelt, and AH Hansen. A LowDimensional Sagittal-Plane Forward-Dynamic Model for Asymmetric Gait and Its Application to Study the Gait of Transtibial Prosthesis Users. Journal of Biomechanical Engineering, 131, 2009b.

J. L. Su and J. B. Dingwell. Dynamic stability of passive dynamic walking on an irregular surface. Transactions of the ASME, 129:802-810, December 2007.

M. Vukobratović, B. Borovac, D. Surla, and D. Stokic. Biped Locomotion. Springer-Verlag, Berlin, Germany, 1990.

M. Vukobratović, B. Borovac, and V. Potkonjak. ZMP: A review of some basic misunderstandings. International Journal of Humanoid Robotics, 3(2):153-175, June 2006.

Q. F. Wei, P. S. Krishnaprasad, and W. P. Dayawansa. Modeling of impact on a flexible beam. In 32nd IEEE Conference on Decision and Control, San Antonio, TX, December 1993.

E. Wendel and A. D. Ames. Rank properties of Poincaré maps for hybrid systems with applications to bipedal walking. In Hybrid Systems: Computation and Control, Stockholm, Sweden, 2010.

E. Westervelt, J.W. Grizzle, and D.E. Koditschek. Hybrid zero dynamics of planar biped walkers. IEEE Transactions on Automatic Control, 48(1):42-56, January 2003.

E. R. Westervelt, G. Buche, and J. W. Grizzle. Experimental validation of a framework for the design of controllers that induce stable walking in planar bipeds. International Journal of Robotics Research, 24(6):559582, June 2004.

E. R. Westervelt, J. W. Grizzle, C. Chevallereau, J.-H. Choi, and B. Morris. Feedback Control of Dynamic Bipedal Robot Locomotion. Control and Automation. CRC Press, Boca Raton, FL, June 2007.

M. Wisse and R. Q. van der Linde. Delft Pneumatic Bipeds, volume 34 of Springer Tracts in Advanced 
Robotics. Springer-Verlag, Berlin, Germany, 2007.

M. Yagi and V. Lumelsky. Synthesis of turning pattern trajectories for a biped robot in a scene with obstacles. In IEEE/RSJ Conference on Intelligent Systems and Robots, pages 1161-1166, Takamatsu, Japan, 2000.

J. Yamaguchi, A. Takanishi, and I. Kato. Experimental development of a foot mechanism with shock absorbing material for acquisition of landing surface position information and stabilization of dynamic biped walking. IEEE International Conference on Robotics and Automation, 3:2892-2899, May 1995.

T. Yang, E. R Westervelt, and A. Serrani. A framework for the control of stable aperiodic walking in underactuated planar bipeds. In IEEE International Conference on Robotics and Automation, pages 4661-4666, Rome, Italy, 2007.

T. Yang, E. R. Westervelt, A. Serrani, and J. P. Schmiedeler. A framework for the control of stable aperiodic walking in underactuated planar bipeds. Autonomous Robots, 27(3):277-290, 2009.

H. Ye, A. N. Michel, and L. Hou. Stability theory for hybrid dynamical systems. IEEE Transactions on Automatic Control, 43(4):461-474, April 1998.

F. Zhang. The Schur Complement and Its Applications. Springer, New York, NY, 2005. 